\title{
The Effects of Parent-Child Interaction Therapy on Symptoms and Impairment in Young Children with Attention-Deficit/Hyperactivity Disorder
}

Stephanie M. Wagner
West Virginia University

Follow this and additional works at: https://researchrepository.wvu.edu/etd

\section{Recommended Citation}

Wagner, Stephanie M., "The Effects of Parent-Child Interaction Therapy on Symptoms and Impairment in Young Children with Attention-Deficit/Hyperactivity Disorder" (2011). Graduate Theses, Dissertations, and Problem Reports. 4811.

https://researchrepository.wvu.edu/etd/4811

This Dissertation is protected by copyright and/or related rights. It has been brought to you by the The Research Repository @ WVU with permission from the rights-holder(s). You are free to use this Dissertation in any way that is permitted by the copyright and related rights legislation that applies to your use. For other uses you must obtain permission from the rights-holder(s) directly, unless additional rights are indicated by a Creative Commons license in the record and/ or on the work itself. This Dissertation has been accepted for inclusion in WVU Graduate Theses, Dissertations, and Problem Reports collection by an authorized administrator of The Research Repository @ WVU.

For more information, please contact researchrepository@mail.wvu.edu. 
The Effects of Parent-Child Interaction Therapy on Symptoms and Impairment in Young Children with Attention-Deficit/Hyperactivity Disorder

\author{
Stephanie M. Wagner
}

Dissertation submitted to the Eberly College of Arts and Sciences

at West Virginia University in partial fulfillment of the requirements

for the degree of

Doctor of Philosophy

in

Psychology

Cheryl B. McNeil, Ph.D., Chair Elisa Krackow, Ph.D.

Tracy L. Morris, Ph.D.

Claire St. Peter-Pipkin, Ph.D.

Christina Wilson, Ph.D.

Department of Psychology

Morgantown, West Virginia

2011

Keywords: Parent-Child Interaction Therapy; PCIT, Behavioral Parent Training; AttentionDeficit/Hyperactivity Disorder; ADHD; Externalizing Disorders 


\begin{abstract}
The Effects of Parent-Child Interaction Therapy on Symptoms and Impairment in Young Children with Attention-Deficit/Hyperactivity Disorder
\end{abstract}

Stephanie M. Wagner

Attention-Deficit/Hyperactivity Disorder (ADHD) is an externalizing disorder beginning in childhood with symptoms and impairment persisting into adulthood for many individuals. Although ADHD is identifiable and diagnostically valid during the preschool years, the majority of the research has focused on treating school-aged children. Some research suggests that behavioral parent training (BPT) programs are efficacious in treating young children. Yet, results are inconsistent and previous research has methodological limitations that restrict interpretations of the data. Parent-Child Interaction Therapy (PCIT), a widely-disseminated evidence-based BPT program for young children with disruptive behavior problems, incorporates components (e.g., in vivo coaching) that may be particularly effective for this population. Therefore, the current study investigated the effects of PCIT on ADHD symptoms and impairment utilizing a non-concurrent multiple baseline design across 4 children (ages 5 and 6). Results demonstrated a visible increase in child on-task behavior in 50\% of the children, a decrease in maternal report of child ADHD symptoms, impairment, and ODD symptoms, and maternal report of treatment satisfaction. These findings suggest that PCIT may reduce attention and hyperactivity/impulsivity problems in addition to oppositional behaviors. Despite these promising results, the inconsistent findings pertaining to on-task behavior, persistence of school difficulties and parenting stress demonstrate the need for further intervention. Additionally, limitations pertaining to the design, sample, and measurement are discussed. 


\section{Acknowledgement}

I would to extend my sincere appreciation to a number of people who supported and helped me during this project and my graduate studies.

I thank my dissertation committee chair and graduate advisor, Dr. Cheryl McNeil. I attribute much of my success in completing this project and in my career thus far to her support, encouragement, and guidance. I feel very lucky to have worked with her during these last six years. Not only, did Dr. McNeil serve as a wonderful academic mentor, she also taught me how to balance work and life.

Additionally, I would like to thank my dissertation committee, Dr. Elisa Krackow, Dr. Tracy Morris, Dr. Claire St. Peter-Pipkin, and Dr. Christina Wilson, for their input and assistance on this project. The committee provided valuable input regarding the literature reviewed, study design, measurement, and graphical displays of results.

Further, I owe a huge thanks to my co-therapists, Ashley Tempel and Andrea Jones, who volunteered to work on a home-based treatment study, which resulted in numerous road trips throughout North Central West Virginia. I appreciate both of their willingness to help out in both rain and shine. They also helped me think on the spot and problem-solve issues that arose over the course of conducting therapy in participants' homes.

I also am thankful of the families who served as participants. All of these families had significant stressors and hectic lives. I am appreciative of their dedication to the project and their child's treatment. I could not have completed this project without them!

Also, I am truly grateful to have wonderful family members and friends who supported me through this journey. I am grateful my parents and grandparents who valued higher education, perseverance, and a strong work ethic. Family supported me both emotionally and at 
times financially during these years. Last but certainly not least, I am so appreciative of my amazing friends who provided both social support and comedic relief necessary to successfully complete a doctorate. 
Table of Contents

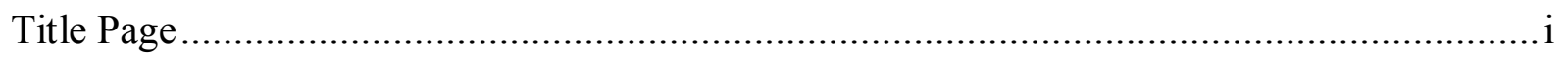

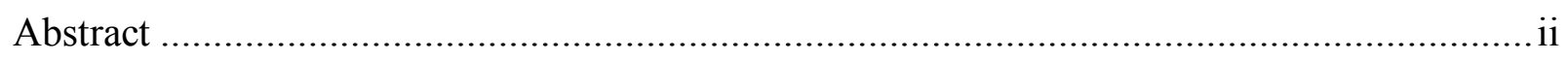

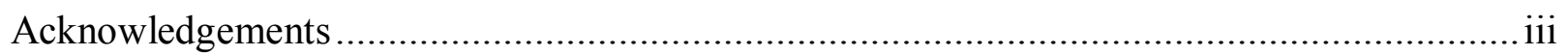

Table of Contents ...............................................................................................

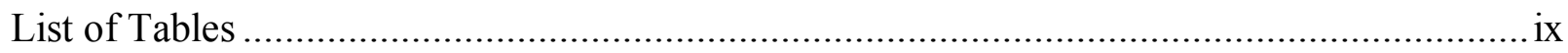

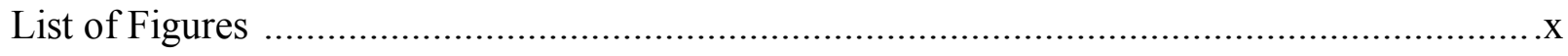

Literature Review ............................................................................................. 1

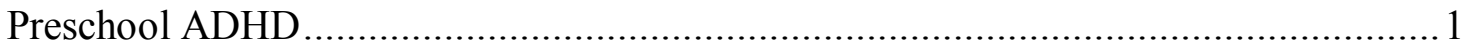

Course of ADHD ................................................................................. 3

Empirically-Supported Treatments ....................................................... 4

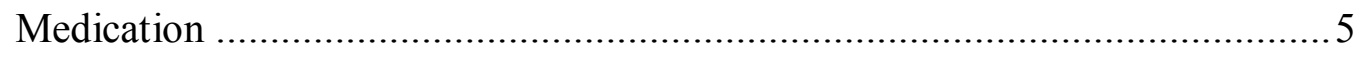

Behavior Modification (Parent Training) …........................................ 5

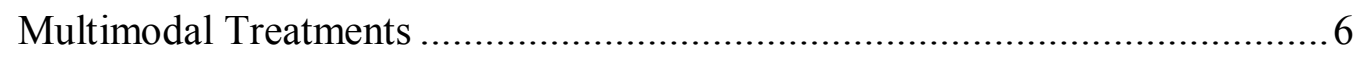

Safety and Long-term Efficacy ..................................................... 9

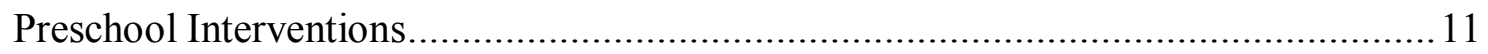

Parent-Child Interaction Therapy .................................................. 15

Empirical Support ................................................................. 18

Similarities to Parent Training for ADHD ...................................... 19

Unique Components that May Target ADHD Symptoms and Impairment

Inclusion of Children with ADHD and Outcomes ...........................22

Statement of the Problem and Hypotheses ................................................................. 30 
Hypotheses (Observable Behavior) ............................................................... 31

Exploratory Questions (Standardized Rating Scales) ........................................ 31

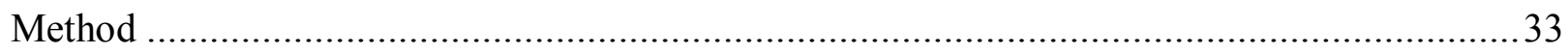

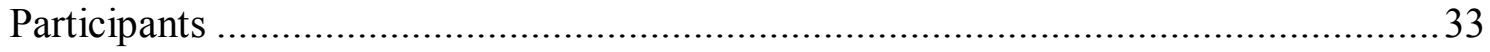

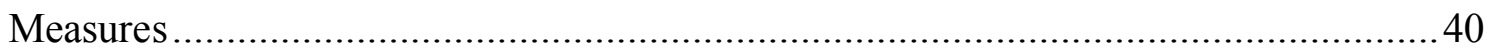

Conners' Parent Rating Scale - Revised Short Version.............................. 40

Conners' Teacher Rating Scale - Revised Short Version ..............................40

Demographics From ......................................................................... 41

DSM-IV Structured Interview for Disruptive Behavior Disorders .................. 41

Dyadic Parent-Child Interaction Coding System-III.................................. 42

Eyberg Child Behavior Inventory ....................................................... 43

Impairment Rating Scale ............................................................. 44

Parenting Stress Index —Short Form.................................................... 44

Revised Edition of the School Observation Coding System (on-task behavioral

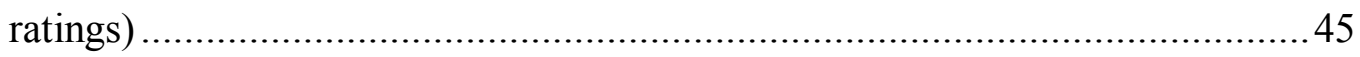

Therapy Attitude Inventory ........................................................... 46

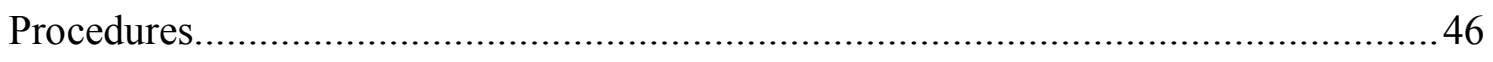

Design Considerations ........................................................................ 46

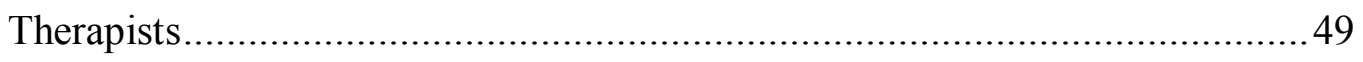

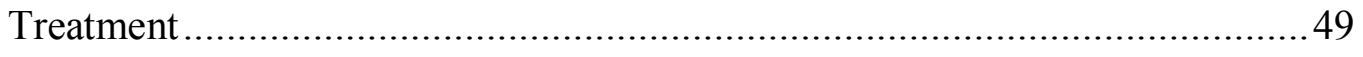

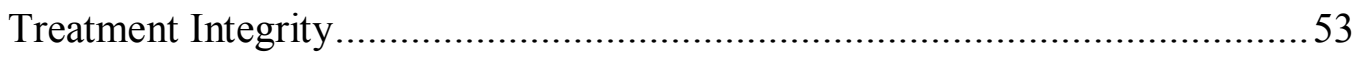

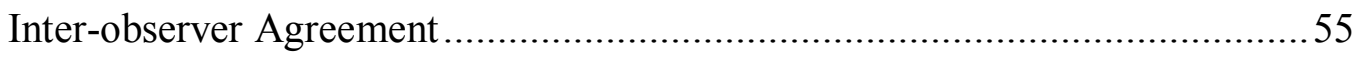

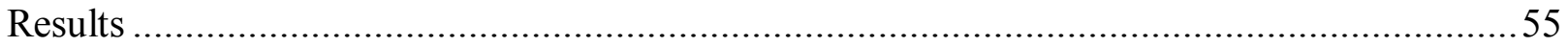




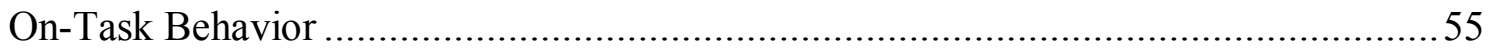

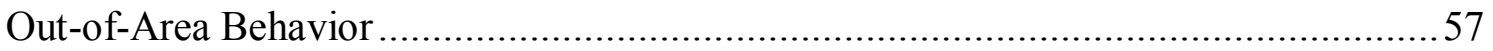

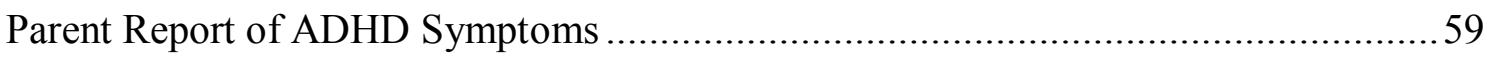

Conners' Parent Rating Scale - Revised Short Version .....................59

DSM-IV Structured Interview for Disruptive Behavior Disorders .......60

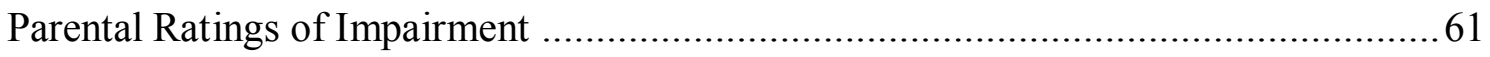

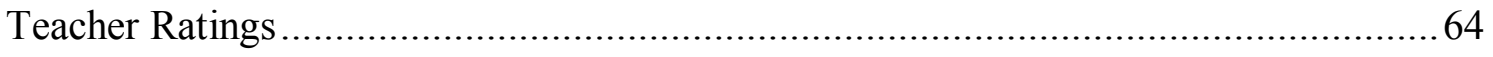

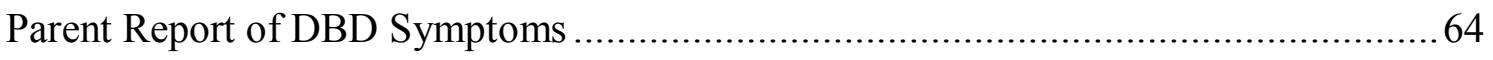

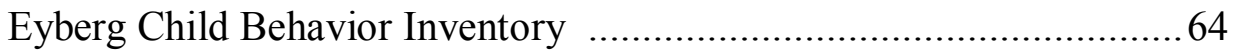

Conners' Parent Rating Scale - Revised Short Version ................... 66

DSM-IV Structured Interview for Disruptive Behavior Disorders ........67

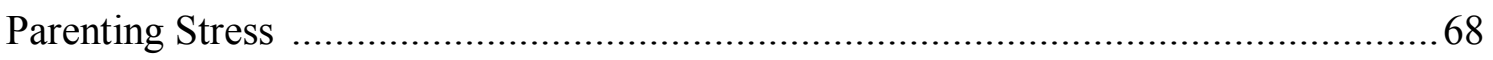

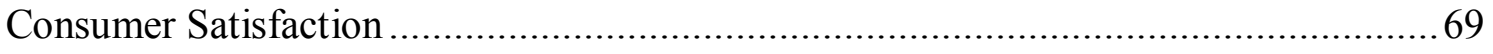

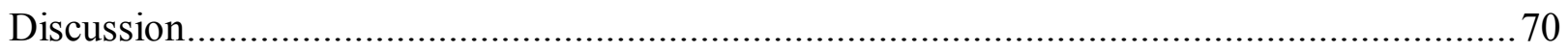

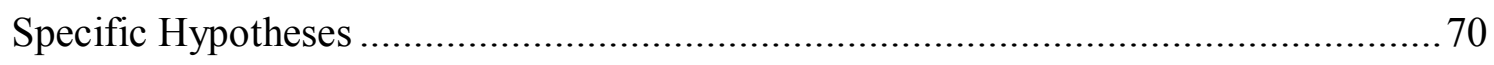

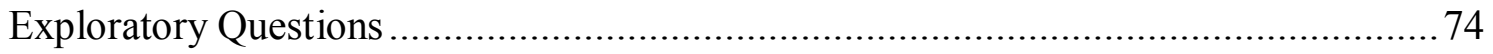

Additional Limitations ............................................................................. 83

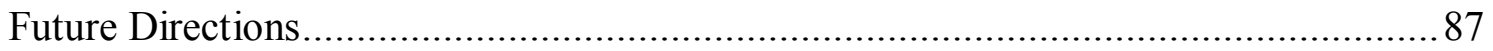

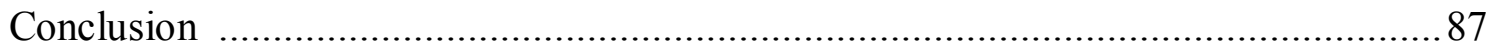

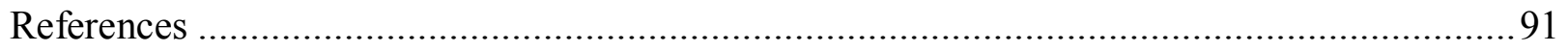

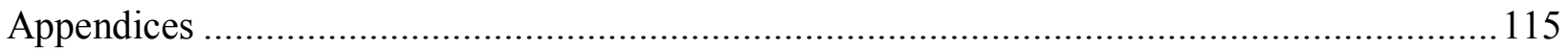

A - Definitions of DPICS-III Codes for the Study …..................................... 115

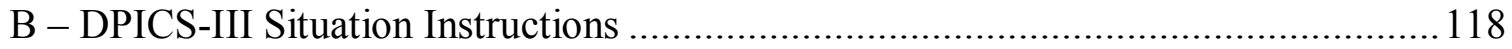




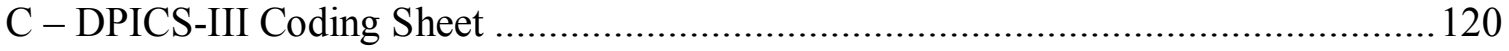

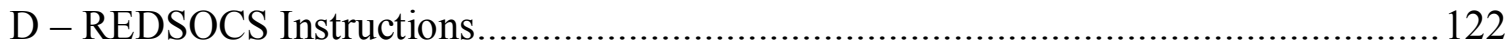

E - REDSOCS Record Form ...................................................................... 124

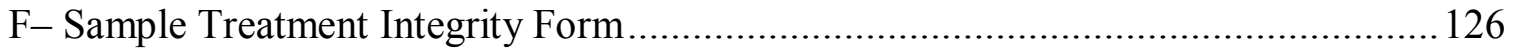




\section{List of Tables}

Table 1. Treatment Length for Current Study and Past PCIT Research ................................ 131

Table 2. Comparison of ECBI Intensity and Problem Scores to Previous PCIT Studies ........... 132

Table 3. Comparison of Consumer Satisfaction to Previous PCIT Studies ............................ 133 


\section{List of Figures}

Figure 1. Percent of time spent on-task during coloring for each participant across phases...... 134

Figure 2. Percent of time spent out-of-area during CDI for each participant across phases ...... 135

Figure 3. Conners' Parent Rating Scale - Revised Short Version (CPRS-RS) Subscales across

participants and assessment points with horizontal lines indicating clinical cutoff ................ 136

Figure 4. DSM-IV Diagnostic Interview for Disruptive Behavior Disorder Subscales across

participants and assessment points.

Figure 5. Impairment Rating Scale (IRS) Subscales across participants and assessment points

with horizontal lines indicating clinical cutoff.

Figure 6. Eyberg Child Behavior Inventory (ECBI) Subscales across participants and assessment points with horizontal lines indicating clinical cutoff.

Figure 7. Parenting Stress Index - Short Form (PSI-SF) Overall Scale and Subscale across

participants and phases with phase lines indicating clinical cutoff. 


\section{Literature Review}

Attention-deficit/hyperactivity disorder (ADHD) is an externalizing disorder beginning in childhood that can be further divided into three distinct subtypes: Predominantly Inattentive, Predominantly Hyperactive/Impulsive, and Combined (American Psychiatric Association, 2000). All three subtypes have characteristic symptoms of developmentally inappropriate levels of inattention and/or hyperactivity/impulsivity that are present in at least two settings (APA, 2000). ADHD is not only one of the most common mental health disorders of childhood but also is one of the most frequent reasons for referral to a mental health professional (Barkley, 1998). Prevalence rates and estimates vary, but ADHD likely effects about 3-7 \% of the population (APA, 2000). Additionally, many children with ADHD meet diagnostic criteria for a comorbid Disruptive Behavior Disorder (DBD). Both Oppositional Defiant Disorder (ODD) and Conduct Disorder (CD) are DBDs that often co-occur with ADHD (e.g., August, Realmuto, MacDonald, \& Nugent, 1996; Pelham, Gnagy, Greenslade, \& Milich, 1992). Overall, ADHD symptoms and symptoms of comorbid DBDs cause substantial problems across many areas of functioning such as academic achievement, peer and sibling relationships, self-esteem, and relationships with adults (i.e., parents and teachers) (APA, 2000). The current literature review covers assessment and diagnostic issues in young children, the course of ADHD, empirically-supported treatments for children (i.e, medication, behavior modification, and combined treatments), and concludes with preschool behavioral parent training outcome research with an emphasis on PCIT research.

\section{Preschool ADHD}

ADHD research has focused more on school-aged children compared to other age groups. In addition to the emphasis on school-aged children, research examining ADHD in other age groups faces unique challenges because the DSM-IV diagnostic criteria are not particularly 
developmentally sensitive for younger and older individuals (Pelham, Chacko, \& Wymbs, 2004). For instance, most preschoolers, even ones without ADHD, can be described using DSM-IV criteria such as - n the go" (Pelham et al., 2004). Although there are specific challenges in diagnosing ADHD in younger children, research demonstrates that many children with ADHD can be accurately diagnosed before age 6 (e.g., Barkley, 1997; Campbell, 1995; Campbell, 2002; Lavigne et al., 1996). Even though the accuracy of diagnosis is higher for children who can be diagnosed in a structured environment (e.g., school setting; Pelham et al., 2004), research shows that the diagnosis is valid during the preschool years especially for older preschoolers (Lahey et al., 1998). Research showing similar prevalence rates in young children and school-aged children (ranging from 2 to 10\%) adds further support for the validity of the diagnosis in this age group (Pelham et al., 2004).

Research also indicates that symptoms of inattention and hyperactivity/impulsivity persist into elementary school in children who are initially identified in preschool (e.g., Cantwell \& Baker, 1989). von Stauffenberg and Campbell (2007) found that ADHD symptoms are moderately stable over time and that behavioral inhibition and inattention, as measured on neuropsychological tests at 54 months, predicted ADHD symptoms in third grade. In addition to the short term stability of symptoms, Pierce, Ewing, and Campbell (1999) followed two cohorts of preschool-aged children into early adolescence. These researchers found children identified as hard to manage" in the preschool years were more likely to meet criteria for an externalizing disorder (ADHD, ODD, and/or CD) at age 13 when compared to other children. Moreover, the persistence of problems into early adolescence was greater for individuals whose problems remained stable from the preschool years to elementary school (Pierce et al., 1999). 
Although the persistence of early symptoms of inattention and hyperactivity/impulsivity appears to be moderately stable, less research has examined the persistence of associated areas of functional impairment and whether the presence of early symptoms predicts later associated problems. Lahey and colleagues (2004) examined ADHD symptoms and impairment in 255 children with and without ADHD (ages 3-7) over the course of 3 years. Results indicated that children with ADHD at time one were more likely to meet diagnostic criteria for ADHD and were also more likely to exhibit global, social, and academic impairments than comparison children three years later (Lahey et al., 2004). Overall the research on preschool ADHD demonstrates that the disorder is diagnostically valid and both symptoms and impairment persist as children enter formal schooling.

\section{Course of ADHD}

Initially, ADHD was believed to be a disorder of childhood and many thought that children outgrew ADHD symptoms and associated impairment as they aged and matured. However, research did not support this belief and instead demonstrated that ADHD is a chronic disorder that continues to affect the majority of individuals in adolescence and adulthood (Weiss \& Hechtman, 1993). Individuals whose symptoms persist over time are at risk for numerous negative outcomes. Some of the more severe aversive outcomes include criminal activity and incarceration (Lynam, 1996) and substance abuse and dependency (Murphy \& Barkley, 1996). In evaluating risk for adult criminal activity, research suggests that individuals with co-occurring conduct problems and ADHD are at a heightened risk, and the presence of ADHD without conduct problems may not increase the risk of adult criminality above the risk in a comparison group (Satterfield, Faller, Crinella, Schell, Swanson, \& Homer, 2007). However, individuals with ADHD are also more likely to have problems in adaptive areas of functioning. When 
compared to adults who do not have ADHD, individuals with chronic ADHD are also at increased risk for adaptive problems such as employment difficulties, marital difficulties, social problems (e.g., fewer close friends and more difficulties keeping friends than comparison adults), and problems with risky sexual activity and early parenthood (Barkley, Fischer, Smallish, \& Fletcher, 2006; Murphy \& Barkley, 1996). Thus, it is clear that individuals who meet criteria for ADHD in childhood are at risk for adverse outcomes including severe problems such as criminal activity and problems in adaptive functioning.

\section{Empirically-Supported Treatments}

Given the persistence of ADHD symptoms and related impairment throughout the lifespan, much research has been conducted to develop and evaluate efficacious interventions. Using large multi-site samples and sophisticated designs, this research has examined many types of treatment including behavior therapies, medication, and combination treatments. Pelham, Wheeler, and Chronis (1998) evaluated the ADHD treatment literature on pharmacological interventions and psychosocial interventions (behavioral parent training and behavioral classroom interventions) according to the APA Task Force criteria for empirically-supported treatments. Utilizing criteria set forth by the task force, Pelham and colleagues (1998) concluded that medication, behavioral psychosocial treatment, and the combination of medication and psychosocial treatments (multimodal treatments) are well-established treatments for children with ADHD. Recently, Pelham and Fabiano (2008) conducted an updated evaluation of evidence-based treatments over the past 10 years (since the initial Task Force), adding intensive peer-focused behavioral treatments (e.g., summer treatment programs) to the list of wellestablished interventions for children with ADHD. 
Medication. Both stimulant medication and atomoxetine (strattera) have been utilized to manage symptoms of ADHD. Stimulant medications have been researched in hundreds of studies and these medications are considered one of the best studied psychiatric pharmacological treatments (Pliszka, 2007). Recently, atomoxetine has been developed as an alternative to stimulant medication, and investigations also support the short-term efficacy of this treatment in reducing ADHD symptoms (Gilchrist \& Arnold, 2008). In recent years, there have been changes in both the prescription and administration of medications with the development of atomoxetine and long-lasting stimulants. For instance, it is now common for children to be prescribed a medication with effects lasting 12 or more hours and it is also more common for children to receive medication 7 days a week (Lerner \& Wigal, 2008).

Behavior modification (parent training). Behavior modification (both classroom and parenting interventions), utilizing behavioral principles and techniques, has been extensively studied (for a review of psychosocial interventions see Chronis, Jones, \& Raggi, 2006). Classroom behavior management has demonstrated efficacy as a treatment for children with ADHD in school settings and some research has found positive changes after academic interventions (Chronis et al., 2006). However, this literature review will solely focus on the parent training literature given its relevance to the current study.

Behavioral Parent Training (BPT) has a long history in treating child disruptive behavior problems. The term encompasses numerous programs designed to teach parents behavior modification for a wide range of ages ranging from preschool-aged children to adolescents (Eyberg, Nelson, \& Boggs, 2008). BPT programs share common components including use of positive reinforcement and consistent discipline techniques (Kaminski, Valle, Filene, \& Boyle, 2008; Shanley \& Niec, 2010); however, specific programs vary in format (e.g., group versus 
individual) mode of delivery (e.g., videotape modeling, in session practice), timing of therapist feedback (e.g., in vivo, delayed), and type of reinforcer (e.g., token economies with tangible reinforcers, social reinforcers) (Kaminski et al., 2008).

BPT has received much attention from researchers in the field who have examined its efficacy for samples of school-aged children with ADHD (for review see Chronis et al., 2006). Chronis, Chacko, Fabiano, Wymbs, and Pelham (2004) conducted a comprehensive review of the parent training literature for child ADHD and found that 28 parent training studies specifically addressed this population. An examination of these studies revealed positive changes in observed and reported child ADHD symptoms following treatment and some evidence of treatment generalization to other contexts. Although this review included research utilizing samples of children ages 3 to 14 , the mean child age was 7.9. Recent efficacy research (Danforth, Harvey, Ulaszek, \& McKee, 2006) and effectiveness (van den Hoofdakker, van der Veen-Mulders, Sytema, Emmelkamp, Minderaa, \& Nauta, 2007) suggest that parent training has utility in treating school-aged children with ADHD. Research has found different effects following parent training with some research demonstrating efficacy in reducing co-occurring problems (behavioral and/or internalizing) (e.g., van den Hoofdakker et al., 2007) and other research finding a decrease in ADHD symptoms and parental stress following parent training (e.g., Danforth et al., 2006).

Multimodal treatments. Although research has demonstrated the efficacy of both medication and behavioral interventions alone, much recent focus has been given to multimodal or combined treatments. To date, the largest randomized clinical trial comparing empiricallysupported treatments (stimulant medication, behavioral treatment, combined treatment, and community care as usual) for school-aged children (ages 7 to 9) has been the Multimodal 
Treatment Study for Children with ADHD (MTA; The MTA Cooperative Group, 1999). In the MTA, parent training was not examined in isolation but rather was a component of a package also including, school consultation and a Summer Treatment Program for the children. The parent training utilized was a combination of programs developed by Barkley and Forehand and McMahon, and parents received a total of 35 sessions of parent training (27 group and 8 individual sessions; The MTA Cooperative Group, 1999). This parent training also incorporated several components that are not typically included in standard parent training including ways to manage stress and anger, techniques for parents to help their child with peer interactions, and skills for interacting with school systems (Wells et al., 2000). Content in individual and group sessions consisted of didactic presentation, modeling, role-playing, group discussion (group sessions only), and assessing and problem solving skill acquisition (individual sessions only) (Wells et al., 2000).

Findings from the MTA were not particularly favorable with regard to behavioral interventions overall (which included parent training). Specifically, findings indicated that medication alone was superior to behavioral treatment alone (The MTA Cooperative Group, 1999). Additionally, study results suggested that adding behavioral treatment to medication did not significantly enhance treatment gains compared to the gains detected in the medication alone group (The MTA Cooperative Group,1999). However, combined treatment did slightly outperform medication treatment alone for children with co-occurring ODD/CD and children with a comorbid internalizing disorder (Jensen et al., 2001). It is important to note that children enrolled in the MTA were older than preschoolers and that the parent training component did not include individual data-driven progression (mastery criteria) and did not incorporate coaching at the same intensity as PCIT (Kaminski et al., 2008). Overall, the interpretation of the MTA 
results has been debated and many critics have cited both methodological limitations of the study as well as practical considerations when utilizing this research to inform clinical practice (e.g., Hoza, Kaiser, \& Hurt, 2007; Pelham, 1999).

Hoza and colleagues (2007) reviewed the literature on multimodal treatment outcome research to highlight how differential outcomes in the literature can be explained based on differential designs. For instance, the authors discuss how the administration sequence of treatments (medication and psychosocial) may result in different outcomes. Specifically, when medication is administered first, behavioral treatments may not show significant incremental effects (e.g., Klein, Abikoff, Hetchman, \& Weiss, 2004). However, when medication and behavioral treatments are started simultaneously, research suggests that combined treatment results in small incremental gains over medication treatment alone (e.g., Klein \& Abikoff, 1997). Additionally, children in the MTA who received combined treatment were successfully managed on a lower dose in comparison to children receiving medication in isolation (Vitiello et al., 2001). Moreover, almost three quarters of the group receiving behavioral treatment without medication were managed successfully over the 14 months of active treatment (Swanson et al., 2001). Other issues that may account for outcome variability include the use of treatment packages with multiple components without an understanding of the effectiveness of individual components and differences in results and effect sizes within studies depending on assessment method (Hoza et al., 2007). Therefore, although behavioral treatments were not as effective as medication alone or combined treatments in the MTA study, it is plausible that characteristics of the design may have influenced these findings.

In addition to design issues of the MTA, Pelham (1999) has raised other concerns in regard to using findings from the MTA as rationale for only using medication to treat ADHD. 
These limitations include the following: (a) roughly $30 \%$ of children do not respond to medications, (b) medications do not cause long-lasting changes and only work while the child is taking them, and (c) while medications are effective in reducing ADHD symptoms, medications do not target associated impairment in children with ADHD such as problems with peer and family relations (Pelham, 1999). Also, problems with adherence to medication in the MTA were identified through objective assessment with saliva samples (Pappadopulos et al., 2009). Pappadopulos and colleagues (2009) found that parents were inconsistent reporters and that behavioral treatment helped mitigate the effects of a missed dose. An additional argument for psychosocial treatments is that parents report greater consumer satisfaction with behavioral treatments, which may result in increased motivation to continue these treatments over a longer period of time (Pelham, 1999). It is possible that parents report less satisfaction with stimulant medication because they have to deal with their child's symptoms of inattention and hyperactivity/impulsivity during times when the child is likely not taking medication (i.e., during morning and evening routines; Chronis et al., 2004). Nevertheless, consumer satisfaction is important when making treatment decisions for chronic disorders.

Safety and long-term efficacy. In addition to design issues of the MTA and practical limitations of medication, another concern regarding ADHD treatments is the safety and efficacy of treatment over time. Long-term safety and efficacy is imperative given the chronic nature of ADHD. Although the majority of research on safety has been conducted on medication, there has been some discussion of safety in psychosocial interventions. Evans, Schultz, and Sadler (2008) reviewed the literature on psychosocial treatments for ADHD including daily report cards, parent training, homework management plans, organization interventions, and social interventions (i.e., social skills training programs). They emphasized that there is a paucity of 
research on the safety of various psychosocial techniques. Evans and colleagues suggested several potential risks associated with psychosocial interventions including the training and social reinforcement of delinquent behavior that is a unique concern in group social skills training (Dishion, McCord, \& Poulin, 1999) as well as the possibility of poor implementation of behavioral techniques either by parents or unskilled professionals (i.e., parents who attest that timeout does not work for their child or professionals who do not understand behavioral principles and are not able to successfully implement the intervention; Evans et al., 2008).

The safety and long-term efficacy of stimulant medication has been called into question in the literature much more than the safety of psychosocial interventions. Gilchrist and Arnold (2008) reviewed the literature on pharmacological treatments for ADHD and noted that, although research supports the short-term efficacy of stimulant medications and atomoxetine, less is known about the long-term effects. These authors noted that research demonstrates efficacy in decreasing ADHD symptoms for up to 15 months with additional preliminary evidence of treatment benefits persisting for 2 years. Yet, literature on the effects of stimulant medication after 2 years is lacking. Gilchrist and Arnold (2008) noted that the MTA study confirmed that methylphenidate is an efficacious treatment over the course of 14 months. The findings in the MTA study are consistent with previous research using a similar time period between initial treatment and post-treatment data collection. The follow-up evaluations of children in the MTA study demonstrated that the medicated groups (the combined group and the medication group) exhibited fewer symptoms than the community group due to the increased medication use in these groups (The MTA Cooperative Group, 2004). This result was evident in the 2-year followup; however, by the 3-year follow-up, these differences between the community group and medicated groups had dissipated (Jensen et al., 2007). Additionally, 8 years following the MTA, 
participants who completed treatment (i.e., all study conditions including the community group) did not differ and exhibited worse outcomes in many areas including delinquency and arrests, academic achievement and grade retention compared to local comparison children without ADHD (Molina et al., 2009). Further by this follow up, 62\% percent of participants who took medication at post-treatment had stopped this treatment (Molina et al.,2009). Poor adherence to medication after 14-months prevents conclusions about the long-term effects but raises questions about the feasibility of long-term pharmacological treatment.

Safety is another important consideration when evaluating pharmacological interventions. Typically-reported side effects associated with stimulant medication include: difficulty falling asleep, decreased appetite, abdominal pain, weight loss, tics, feeling jittery, and headaches (Wolraich, McGuinn, \& Doffing, 2007). In a review of the empirical research on stimulant medications, Lerner and Wigal (2008) concluded that empirical support for claims of serious side effects from stimulant medication is lacking in the research. They noted that side effects such as appetite loss, short term weight loss, and small increases in blood pressure and heart rate are typically only clinically meaningful in a small percentage of individuals (Lerner \& Wigal, 2008).

In general, there is a well of research on interventions for childhood ADHD with empirical support for medication, behavioral treatments (both classroom interventions and behavioral parent training), combined treatments, and intensive peer interventions. While results of the MTA study support medication and combined treatments over behavioral treatment in isolation, methodological issues as well as questionable long-term adherence, efficacy, and safety highlight the need for behavioral or combined treatments. Accordingly, early intervention during the preschool years may be particularly beneficial.

\section{Preschool Interventions}


Although it is clear that there is considerable research support for ADHD treatment in school-aged children, fewer investigations have focused exclusively on preschoolers. However, developing and testing early interventions, is extremely important given the early age of onset and chronic nature of ADHD. Research has examined the efficacy of medication and behavioral interventions in efforts to develop methods to prevent negative outcomes later in life.

There are more potential concerns when treating young children with medication alone compared to treating other age groups. Historically, there has been substantially less research on the use, effectiveness, and safety of stimulant medications with preschoolers, and the existing research has had significant methodological problems and yielded inconsistent findings (Greenhill et al., 2006). Furthermore, atomoxetine has not been researched in children younger than age 6 (Kratochvil et al., 2006). Therefore, the Preschool ADHD Treatment Study (PATS) was specifically designed and conducted to clarify inconsistencies in the literature on stimulant medications utilizing a large multi-site design. In this study, parents of young children first received parent training to learn to manage their child's challenging behaviors and then only progressed to the medication trial if the child did not improve from parent training and if the parents consented to the research. The parent training model utilized in PATS was derived from the Cunningham (e.g., Cunningham, Bremner, \& Boyle, 1995) group program, which consisted of ten 2-hour sessions, and did not incorporate either coaching (Kaminski et al., 2008) or datadriven individualized progression. The overwhelming majority of children were enrolled in the medication trial and results from this trial demonstrated statistically significant decreases in symptoms of inattention and/or hyperactivity/impulsivity. Although these findings showed a reduction in symptoms, the effect sizes were smaller than the effects observed in the MTA study of school-aged children (Greenhill et al., 2006). Findings also demonstrated that most children 
tolerated the medication without severe side effects (Greenhill et al., 2006); however, medication did significantly restrict physical growth (Swanson et al., 2006). Additionally, it is important to note that a higher percentage of preschoolers in PATS discontinued medication as a result of adverse side effects than school-aged children in the MTA (Lerner \& Wigal, 2008). Therefore, although medication is an efficacious treatment for preschoolers with ADHD, the general limitations of medication as well as specific concerns regarding medicating young children, necessitate empirical examination of the use of psychosocial treatments (e.g., parent training) with this population.

Researchers have noted the paucity of research on BPT for preschoolers with ADHD in comparison to the abundance of research with school-aged children (e.g., Sonuga-Barke, Daley, Thompson, Laver-Bradbury, \& Weeks, 2001). This gap in the literature was surprising given that a relation between behavioral problems and maladaptive parenting practices exists in parents of preschoolers with ADHD (Mash \& Johnston, 1982) and because intervening early has the potential to prevent initial problems from escalating (McGoey, Eckert, \& Dupaul, 2002). Also, findings indicate that children with ODD or CD with comorbid ADHD or hyperactivity problems respond as favorably to BPT as children with DBDs without comorbid ADHD (e.g., Hartman, Stage, \& Webster-Stratton, 2003).

Early research with preschoolers demonstrated inconsistent findings in terms of initial decreases in ADHD symptoms and maintenance of these improvements over time (i.e., Erhardt \& Baker, 1990; Pisterman et al., 1989; Strayhorn \& Weidman, 1989, 1991). These findings may be the result of methodological differences in measurement of ADHD symptoms and in differences of format and delivery of parent training. McGoey and colleagues (2002) reviewed these studies noting major limitations in many, including: the failure to measure related child 
behavior problems, an absence of long-term follow-up data, and not examining the generalization of treatment effects.

Given the limitations of early studies and the recent call for research in the area, several studies have examined the effects of research-supported parenting interventions (i.e., Triple $\mathrm{P}$ Positive Parenting Program and Incredible Years Basic Parent Training) on symptoms of ADHD in young children (i.e., Bor, Sanders, \& Markie-Dadds, 2002; Jones, Daley, Hutchings, Bywater, \& Eames, 2007). Specifically, Jones and colleagues (2007) randomly assigned 79 families of children ages 3 to 4 with scores above the clinical cutoff on a measure of disruptive behavior and a measure of hyperactivity to either the Incredible Years Intervention or a wait-list comparison group. Findings immediately following treatment indicated that ADHD symptoms significantly decreased even after statistically controlling for improvements in child conduct problems and that more children in the treatment group exhibited clinically-significant reductions in ADHD symptoms compared to the wait-list group (Jones et al., 2007). Additionally, Bor and colleagues (2002) randomly assigned 87 families with a child aged 3-4 who met DSM-IV criteria for ADHD based on maternal report and also exhibited elevated conduct problems to either enhanced Triple P, standard Triple P, or a wait-list control group. Results suggested that both Triple $\mathrm{P}$ conditions significantly reduced disruptive behavior and ADHD symptoms compared to the wait-list condition (Bor et al., 2002). However, the study did not find a difference between maternal depression, anxiety, or stress (Bor et al., 2002). Although these results regarding ADHD symptoms are promising, these studies had several limitations. One limitation is that parental report was the primary outcome measure assessing changes in ADHD symptoms (teacher ratings, ratings of impairment and behavioral ratings of on-task behavior were not 
collected; (Bor et al., 2002; Jones et al., 2007), raising questions about how parental expectancies and demand characteristics may have influenced results.

Although the results from research on some preschool parent training programs such as Triple P Positive Parenting Program and the Incredible Years Basic Parent Training are promising, further research with preschoolers is needed given the existing limitations. One intervention that may be useful with this population is Parent-Child Interaction Therapy (PCIT). PCIT is an empirically-supported treatment for young children with DBDs (Eyberg et al., 2008; Eyberg et al., 2001; McNeil \& Hembree-Kigin, 2010). However, to date, no published empirical studies have directly investigated the efficacy of PCIT in treating symptoms and impairment associated with ADHD. Therefore, a description of the treatment, review of what is known about treating children with ADHD, a comparison of PCIT and other programs, and an examination of the inclusion of children with ADHD in PCIT research is warranted to better understand how the treatment may benefit children with ADHD.

\section{Parent-Child Interaction Therapy}

PCIT is a BPT program based on theoretical principles of both social learning theory and attachment theory (Eyberg, 1998). The treatment shares many similarities with other BPT programs as other empirically-supported interventions have derived from Hanf's (1969) twostage model. These parenting programs typically employ a phase targeting positive parenting skills (i.e., praise, parental attention to child) and a discipline phase teaching consistent and firm discipline practices (i.e., parental commands and removal of privileges/timeout). PCIT incorporates these stages in two phases referred to as Child-Directed Interaction (CDI) and Parent-Directed Interaction (PDI). 
Although the theoretical basis and many treatment components of PCIT strongly resemble other BPT programs, it is important to note several unique elements. Specifically, the format and progression through treatment are two distinguishing features. The format of PCIT differs from most other parent training programs because the intervention includes live coaching. Coaching enables both the parent and child to take part in treatment and allows the therapist to observe the interaction, provide feedback, and assist the parent in difficult situations. Progression through treatment also distinguishes PCIT from most other programs because a set number of sessions is not prescribed at the onset of treatment. Instead, parents must demonstrate mastery of skills to progress through and, eventually, complete treatment (Bahl, Spaulding, \& McNeil, 1999). The observation of parental skills throughout treatment also enables the therapist to target specific challenging areas based on individual families' strengths and weaknesses.

The first phase of PCIT, CDI, shares many similar characteristics with traditional play therapy and functionally is designed to improve the parent-child relationship. During this phase, parents learn specific skills that enable the child to direct the play. Overall goals of this phase are to create or strengthen the parent-child bond, to help parents increase the frequency of positive parenting techniques, and to improve the child's social skills (i.e., prosocial behaviors such as helping and sharing). Skills used in CDI can be remembered with the acronym PRIDE, which stands for: Praising the child (labeled praise), Reflecting the child's verbalizations, Imitating the child's play, Describing the child's behavior, and using Enthusiasm throughout the play. Parents also receive instruction on avoiding certain behaviors, including: questions, commands (both direct and indirect), and criticisms. Parents are instructed in differential or selective attention. They are taught a specific procedure to ignore mildly inappropriate or negative behaviors while praising the opposite or incompatible appropriate behavior (i.e., a 
parent would ignore a child's whining and then give a labeled praise when the child uses a big boy" or big girl" voice). All of these skills are practiced extensively in therapy sessions with the therapist coaching the parents and providing constructive feedback. The skills also are practiced at home between sessions. Progression to the second phase of treatment, PDI, depends on how quickly parents acquire and demonstrate these skills. Specifically, parents must demonstrate mastery by giving 10 labeled praises, 10 behavioral descriptions, and 10 reflections in a 5-minute play interaction. During this interaction, the parents must have a total of three or fewer questions, commands, and criticisms.

After mastering skills taught in CDI, families start the discipline phase of treatment, PDI. The goals of this phase are to improve parental discipline techniques through the use of a firm, consistent, and predictable procedure and to increase child compliance. First, parents learn how to give direct and clear commands and how to determine if the child obeys. They then learn to administer specific consequences contingent on child compliance or noncompliance. If the child obeys the instruction, the parents reward the behavior by giving a labeled praise; however, if the child does not comply, the parent learns how to issue a warning and follow a timeout sequence if necessary. Specifically, if the child has not complied with the command within 5 seconds, parents issue a timeout warning and if the child still does not comply, parents put the child in a timeout chair. A back-up room is utilized for children who do not stay on the chair and serves to teach appropriate timeout behavior. PDI also has specific mastery criteria that determine when therapy can be terminated. Additional topics can be discussed in treatment to promote generalization and maintenance of treatment gains including addressing the occurrence of behavior problems in public settings and setting up house rules for serious problems (e.g., aggressive behavior). 
Empirical support. PCIT has a large evidence-base and numerous studies demonstrate the efficacy of PCIT in reducing the frequency and intensity of disruptive behavior problems in preschoolers. This research has compared clinic-referred children with externalizing problems to both classroom control and waitlist children (e.g., McNeil, Capage, Bahl, \& Blanc, 1999; Schuhmann, Foote, Eyberg, Boggs, \& Algina, 1998; McNeil, Eyberg, Eisenstadt, Newcomb, \& Funderburk, 1991). Research also has demonstrated that treatment gains generalize to other individuals (i.e., untreated siblings; Brestan, Eyberg, Boggs, \& Algina, 1997) and other settings (i.e., school; McNeil et al., 1991). Further, parents completing PCIT report reductions in parenting stress following intervention (e.g., Eisenstadt et al., 1993; Eyberg et al., 2001; McNeil et al., 1999; Schuhmann et al., 1998). Follow-up studies offer support for the maintenance of treatment gains over time (Boggs et al., 2004; Eyberg et al., 2001).

Given the efficacy of PCIT in treating children with disruptive behavior disorders, recent research has focused on utilizing the treatment with new populations. Some of these populations include physically abusive parents (Chaffin et al., 2004), children with separation anxiety (Pincus, Eyberg, \& Choate, 2005), children with depression (Lenze, Pautsch, \& Luby, 2011), children with mental retardation (Bagner \& Eyberg, 2007), and children with autism (Masse, 2009; Masse, McNeil, Wagner, \& Chorney, 2008). In order to comprehensively understand the rationale for examining the effects of PCIT on symptoms of ADHD, it is important to draw parallels between ADHD and parent training programs specifically for ADHD, highlight unique components of PCIT that may enhance outcomes for problems of inattention and/or hyperactivity/impulsivity, and review what is currently known about utilizing PCIT to treat young children with ADHD. 
Similarities to parent training for ADHD. PCIT shares many similarities with parent training programs in general. Although many parent training programs have been utilized with ADHD populations, most were developed to target disruptive behavior problems. Russell Barkley developed a parent training program specifically for treating children with ADHD that is based on similar principles underlying other parent training programs, and his program was one of the ones adapted for use in the MTA. Research supports the efficacy of Barkley's program and studies demonstrated that positive changes have been reported in as many as $64 \%$ of families referred to clinics for their child's ADHD symptoms (Barkley, 2002). A major similarity between Barkley's program and PCIT is that both contain a positive parenting component and a discipline component based on Hanf's (1969) two-stage model for child noncompliance. Also, these treatments teach parents some of the same skills. For instance, both teach parents differential attention (ignoring inappropriate behavior and praising appropriate behavior) and a timeout procedure that addresses how to handle difficult behaviors in timeout.

Although these programs are similar theoretically, the Barkley program and PCIT differ in a number of ways. PCIT was specifically developed for young children; and therefore, is very developmentally-sensitive to the needs of children ages 2 through 7 . Barkley's program was developed for a broader age range, encompassing both preschool-aged and school-aged children (ages 2-12), and may require slight modifications to enhance outcomes with very young children (Barkley, 2002). Also, PCIT is a data-driven program with progress determined by parents' skill acquisition and Barkley's contains 10 steps overall plus a booster session with each individual step typically taking about a single session to complete (Barkley, 2002). Moreover, Barkley's program is more didactic in nature and does not include coaching, which is an integral component of PCIT. 
Barkley's program also incorporates several aspects that are not part of PCIT. Specifically, this program places greater emphasis on the biological underpinnings of the disorder; and therefore, includes a lot of psychoeducation about ADHD. Also, given that this program was developed for a broader age range, the treatment gives parents techniques to deal with their child's behavioral problems in school such as instructions on how to setup a daily report card. PCIT does not typically target school behavior problems because it was developed for younger children, some of whom have not yet started formal education.

The comparison between the Barkley parent training program and PCIT demonstrates many similar components and several differences (i.e., format, target problems, and psychoeducation provided). These similarities increase the likelihood that PCIT will be efficacious for children with ADHD given the efficacy of the Barkley program. However, PCIT also contains many features that may be advantageous in treating children with ADHD.

Unique components that may target ADHD symptoms and impairment. Difficulties in social settings and problems in relationships with peers are characteristic of many children with ADHD (Whalen \& Henker, 1985). These children are less well-liked than their peers (Pelham \& Bender, 1982) and also are more frequently rejected by peers than other children (Hinshaw \& Melnick, 1995), presumably because of their inattentive and hyperactive/ impulsive behaviors (e.g., interrupting other children, not taking turns in games). Research demonstrates that preschoolers with ADHD are less popular, exhibit fewer prosocial behaviors, and demonstrate fewer cooperative behaviors than preschoolers without ADHD (Lahey et al., 1998). These difficulties are particularly concerning because problems in social relationships with peers are predictive of negative long-term outcomes in children with disruptive behavior problems (Coie \& Dodge, 1998). Given the severity of this problem, research has examined different 
approaches to teaching social skills to children with ADHD with mixed results (Pelham et al., 1998). Currently, intensive peer interventions meet criteria for well-established treatment for ADHD (Pelham \& Fabiano, 2008); however, these intensive treatments are often costly and difficult to implement.

Although the overall literature on social skills training is inconsistent, some empirical support exists for interventions that combine social skills training with other treatments such as parent training. In particular, findings from investigations indicate that combining parent training and social skills training may result in larger treatment gains and may increase the likelihood of the gains generalizing to other settings (Frankel, Myatt, Cantwell, \& Feinberg 1997; Pfiffner \& McBurnett, 1997; Sheridan, Dee, Morgan, McCormick, \& Walker, 1996). Webster-Stratton and Hammond (1997) examined combined treatments by comparing parent training, social skills training, and a combination of the two and found that the combined treatment was superior to the others in terms of effect size and treatment generalization.

PCIT does not include a component specifically targeting social skills; however, the intervention naturally addresses social skills during sessions. For instance, learning the skills in the context of play allows the parents to model appropriate play skills such as playing gently with the toys, sharing toys, and using polite manners. Additionally, parents learn to use labeled praises to reinforce their child's prosocial behaviors during play (e.g., asking nicely, turn-taking, sharing, playing gently). While parents are praising appropriate behaviors, they are also ignoring socially inappropriate behaviors (e.g., grabbing toys from the parent without asking). This differential attention likely serves to increase the frequency of prosocial behaviors and also decrease inappropriate behaviors during the child's play with the parent, which could then generalize to interactions with peers. 
Diagnostic criteria for ADHD include a number of symptoms of inattention such as difficulty sustaining attention and being easily distracted by extraneous stimuli. In clinical settings during PCIT, children with ADHD frequently lose interest in activities and switch toys often in the beginning of treatment. Additionally, clinical lore suggests that parents of children with ADHD voice frustration that even though they notice inattentive and/or hyperactive/ impulsive symptoms in their child, they also have observed their child sustaining attention for an extended period of time in certain contexts. Specifically, parents often note that their child can focus while playing a video game or engaging in another enjoyable and stimulating activity. In addition to the frustration, parents sometimes are confused by this behavior and may believe that their child can control his or her attention span.

Oftentimes, clinicians explain to parents and other individuals that these behaviors are not inconsistent with an ADHD diagnosis and that theories exist attempting to explain this behavior (i.e., Zentall, 2005). Specifically, a theory of optimal arousal can explain the observed activity level in different organisms, including humans. According to this theory, in order to focus attention and reduce variability in observed behavioral responses, an optimal level of arousal is required (Cooley \& Morris, 1990). The theory of optimal arousal can be applied to individuals with ADHD and is consistent with research on children with ADHD that has demonstrated that they are underaroused and overactive (Rosenthal, 1973). According to this theory, children with ADHD need a greater amount of stimulation compared to other same-age children and that when stimulation in their external environment is insufficient, these children act in ways to stimulate themselves (e.g., daydreaming, talking incessantly or out of turn, exhibiting aggressive behavior etc.; Zentall, 2005). 
Certain characteristics of PCIT may be particularly beneficial for children who need a lot of arousal and stimulation. For instance, several skills that parents learn during the first phase of treatment may help increase sustained attention to task or play. The whole CDI environment is very stimulating when parents fully master the skills (in order to meet mastery criteria, parents must average one positive verbalization every 10 seconds). In addition to the overall CDI environment, behavioral descriptions may improve on-task behavior (Lemaster, Wagner, Tempel, \& McNeil, 2010). Behavioral descriptions are often referred to as running commentary of the child's play and involve the parent describing what the child is doing (Eyberg, Nelson, Duke, \& Boggs, 2005). The use of differential reinforcement in the first phase of PCIT may positively influence sustained attention in children with attention and hyperactive/impulsive difficulties.

Another unique feature of PCIT that may be especially beneficial to families of a young child with ADHD is the data-driven approach of the intervention. Specifically, the stringent criteria to progress in therapy are based on the behavioral principle of over-learning and require the parents to use skills taught at a higher intensity than necessary throughout the day (McNeil \& Hembree-Kigin, 2010). In addition to promoting skill generalization and maintenance, this component of treatment may assist parents in dealing with the variability in their child's inattentive and hyperactive/ impulsive behaviors. Specifically, over-learning may give parents the confidence to handle an unanticipated problematic behavior.

Inclusion of children with ADHD and outcomes. Wagner and McNeil (2008) conducted a critical review of the PCIT outcome literature to investigate the inclusion of children with ADHD, the method of assessing ADHD in research, and the efficacy of PCIT in reducing ADHD symptoms. Results of this review indicated that fourteen published papers on PCIT 
included children with ADHD and that eight studies reported ADHD symptoms before and after treatment. Overall, Wagner and McNeil (2008) concluded that although children with ADHD have been included in PCIT treatment outcome studies, many of these investigations were not directly focusing on ADHD. Therefore, much of the current literature contains methodological problems in the assessment and diagnosis of ADHD, thus limiting conclusions that can be drawn from this existing research. An examination of each of the 14 studies individually demonstrates important strengths and weaknesses.

Six of the fourteen studies initially assessed ADHD symptoms in participants but did not assess ADHD symptoms again at post-treatment or follow-up. Oftentimes, ADHD symptoms were not assessed throughout the study because examining changes in inattention and/or hyperactivity/impulsivity was not a primary objective of the research. Therefore, ADHD was measured initially for the purpose of describing the sample in these studies.

The review of these studies demonstrated problems in assessment methods. Recommendations for evidence-based assessment of ADHD include the integration of clinical interviews, parent and teacher reports on rating scales, behavior observation, and assessment of child impairment in various domains (i.e., relationships with both peers and adults, academic progress, classroom behavior, and family functioning) (Pelham, Fabiano, \& Massetti, 2005). However, four of the studies identified in the review based diagnosis solely on a structured diagnostic interview with a parent and did not include teacher ratings, measures of child impairment, or behavioral observations (i.e., Bagner \& Eyberg, 2003; Brestan et al., 1997; McNeil, Clemens-Mowrer, Gurwitch, \& Funderburk, 1994; Schuhmann et al., 1998). One study utilized teacher ratings and a structured interview with parents (Harwood \& Eyberg, 2004) and one study was a follow-up study in which the method of diagnosis was unclear (Hood \& Eyberg, 
2003). Although overall these studies had problems in the assessment of ADHD, Schuhmann and colleagues (1998) did attempt to address some of the problems in diagnosing young children by using criteria more stringent than the DSM-III-R criteria to diagnose (i.e., the child needed to exhibit ten rather than six symptoms and these symptoms needed to be present for 1-year as opposed to 6 months).

Based on assessments administered in these six investigations, the prevalence of ADHD in PCIT studies varied, ranging from 41\% (Harwood \& Eyberg, 2004) to 78\% (Hood \& Eyberg, 2003). Although ADHD symptoms were not measured both before and after treatment, the studies did find significant decreases in child disruptive behavior as reported on the Eyberg Child Behavior Inventory (ECBI; Eyberg \& Pincus, 1999). These findings suggest that PCIT may effectively treat disruptive behavior problems in children with comorbid ADHD.

Eight of the fourteen studies identified in Wagner and McNeil's (2008) review did assess for ADHD before and after treatment. In general, these studies demonstrated reductions in ADHD symptoms following treatment. However, these studies are also subject to methodological limitations in the initial assessment as well as the choice of measures utilized to assess symptom change (overall lack of multi-method and multi-informant measures). The majority of these studies were not primarily researching the effects of PCIT on ADHD; however, a few of the studies were interested in addressing changes in attention and hyperactivity/ impulsivity following treatment.

Eisenstadt and colleagues (1993) examined the ordering of CDI and PDI in 24 families of a child ages $2 \frac{1}{2}$ to 7 diagnosed with an externalizing disorder (ODD, ADHD, or CD). Based on maternal report on the DSM-III-R Structured Interview for Disruptive Behavior Disorders, 71\% of the children had an ADHD diagnosis. Maternal reports of child behavior on the Werry-Weiss- 
Peters Activity Rating Scale (WWP; Werry, 1968) assessed hyperactivity and attention problems before and after treatment. Post-treatment assessment indicated that PCIT overall, regardless of ordering of CDI and PDI, significantly reduced scores on the WWP Activity Rating Scale.

Eyberg and colleagues (2001) conducted a follow-up study of the 20 families who previously participated in the Eisenstadt and colleagues (1993) study. Seventy five percent of children in these families met diagnostic criteria for ADHD. Thirteen of the 20 families were available for follow-up assessment at 1 and 2 years after treatment. Scores on the WWP Activity Rating Scale at 1-year and 2-year time points were significantly lower than pretreatment scores, indicating that reductions in $\mathrm{ADHD}$ symptoms maintained over time. However, maturational issues confound the interpretation of these findings.

McNeil and colleagues (1991) looked at the generalization of PCIT treatment effects to the school environment by comparing 10 children who completed PCIT, 10 untreated children with behavior problems, and 10 control children without behavior problems. Ninety percent of the treated children exhibited enough symptoms for a DSM-III diagnosis of ADHD. Results demonstrated no significant improvement in percent on task behavior and scores on the Hyperactivity Index of the Conners' Teacher Rating Scale (CTRS; Goyette, Conners, \& Ulrich, 1978) were not significant from pretreatment to post-treatment as compared to controls. Increases in child compliance did generalize to the classroom in this study. Although these results suggest that changes in ADHD symptoms did not generalize to the school, it is unclear as to whether changes in these symptoms occurred at home following PCIT.

Funderburk and colleagues (1998) conducted a 12- and 18-month follow-up evaluation of participants who were originally in the McNeil and colleagues' (1991) study to examine the maintenance of initial results of school generalization by comparing 12 children who had 
received treatment and 72 comparison children. Ninety two percent of the treated children met criteria for ADHD based on the DSM-III-R Structure Interview for DBDs and the treated children were also at least one standard deviation above the mean on the Conduct Problem and/or Hyperactivity Index of the CTRS. Changes in ADHD symptoms were assessed with behavioral observations of on-task behavior and the CTRS. Neither of these measures demonstrated significant and persistent changes in ADHD symptoms in the classroom at followup.

Another follow-up study was conducted by Boggs and colleagues (2004). These researchers examined the long-term outcomes of PCIT by comparing families that completed treatment to families who dropped out 1 to 3 years later. Seventy-two percent of 46 families of a child with ODD ages 3-6 at the time of treatment had co-occurring ADHD based on a maternal report on a DSM-III-R clinical interview. This interview was also utilized to assess ADHD at follow-up and was the only study to repeat a diagnostic interview following treatment. In the treatment completer group, the number of children diagnosed with ADHD decreased from 15 at pretreatment to 8 at follow-up compared to the treatment dropout group in which 18 children were diagnosed at pretreatment and 16 children were diagnosed at follow-up.

In addition to the PCIT studies that have included comorbid ADHD in their sample, some literature has more directly examined attention and/or hyperactivity/impulsivity. For instance, Johnson, Franklin, Hall, and Prieto (2000) reported a PCIT case study of a 7-year old male with a previous ADHD diagnosis who was beginning to exhibit some oppositional behaviors. These authors note observed clinical improvements in the family relationship and also collected some systematic data measuring the boy's behaviors. Specifically, the authors stated that Child 
Behavior Checklist (CBCL; Achenbach, 1991) scores on the Attention Problems and Aggressive Problems subscales decreased but were still elevated at the termination of treatment.

A more systematic study of the effects on PCIT on symptoms of ADHD was conducted by Matos, Torres, Satiago, Jurado, and Rodriguez (2006). These researchers piloted PCIT in nine families of children ages 2-7 using an adapted treatment protocol for Puerto Rican families. This adaptation consisted of translating the treatment manual into Spanish and adding two sessions of psychoeducation. Seventy-eight percent of children enrolled in the study met DSM IV criteria for ADHD based on parental report on the Diagnostic Interview Schedule for Children IV (DISC-IV). Changes in ADHD symptoms were measured with the hyperactivity subscale of the Disruptive Behavior Disorder Scale for Children (DBDRS) and the hyperactivity subscale of the Behavioral Assessment System for Children - Parent Rating Scales (BASCPRS). Statistically significant reductions in hyperactivity on both of these measures were observed.

A more rigorous test of the effects of PCIT on ADHD was conducted by Nixon (2001). Nixon (2001) compared changes in hyperactivity and temperament in preschoolers ages 3-5 who received PCIT compared to a wait-list control group. Based on parental report on the DSM-IV Structured Interview for DBDs, all 34 children met criteria for ODD and $65 \%$ of these children received an ADHD diagnosis. Compared to the wait-list control group, the PCIT group significantly reduced the severity of ADHD symptoms, and fewer children in the PCIT group met diagnostic criteria for ADHD immediately following treatment compared to comparison children. However, maternal reports of ADHD symptoms did not differ significantly between the two groups at a 6-month follow-up. 
Since the review, a couple additional studies examined treatment effects in children with ADHD. McCabe and Yeh (2009) compared a cultural adaptation of PCIT, standard PCIT, and treatment as usual in a sample of 58 Mexican American clinic-referred families with a child age 3-7. ADHD symptoms were assessed using the Early Childhood Inventory (Gadow \& Sprafkin, 1997). Families who received standard PCIT and the cultural adaptation both reported significant reductions in ADHD symptoms compared to the control group. No other ratings of symptoms or impairment were conducted and it is unclear how many enrolled children met diagnostic criteria.

Matos, Bauermeister, and Bernal (2009) randomly assigned 32 families with a child ages 4-6 with ADHD to PCIT or a wait-list control. ADHD symptoms were assessed on the Disruptive Behavior Scale for Children - Spanish (Barkley, Murphy, \& Bauermeister, 1998), the Hyperactivity Subscale of the Behavioral Assessment System for Children - Parent Rating Scale (BASC - PRS - Spanish; Reynolds \& Kamphaus, 1998), and the NIMH Diagnostic Interview Schedule for Children IV - Parent Version (Shaffer, Fisher, Lucas, Dulcan, \& Schwab-Stone, 2000). Matos and colleagues (2009) found clinically-significant changes in hyperactivity on both the DBDRS and Hyperactivity Subscale of the BASC based on maternal report.

Overall, results of the research on PCIT pertaining to young children with ADHD are promising. The current literature suggests that many children presenting to clinical research settings have problems with attention and/or hyperactivity/impulsivity (Wagner \& McNeil, 2008). Some studies have shown significant reductions in symptoms immediately after treatment (e.g., Matos et al., 2009; Nixon, 2001) and a couple of studies have demonstrated longlasting improvements (e.g., Boggs et al., 2004). However, given that assessing and treating ADHD was often not a primary research concern, the current literature reveals many 
methodological weaknesses. Specifically, many studies did not measure clinically-significant changes or report effect sizes and many relied solely on parental report of symptoms (both for the diagnosis and to measure change). Therefore, a direct examination of the effects of PCIT on ADHD employing a multi-method assessment was warranted.

\section{Statement of the Problem and Hypotheses}

Delivering early and effective interventions to children with ADHD is important to decrease the likelihood of adverse outcomes later in life given the persistence of the disorder and its association with severe and adaptive problems. Although empirically-supported treatments exist for children with ADHD, less research has been conducted with preschoolers. Recent investigations have suggested that parent training programs such as the Triple P Positive Parenting Program and Incredible Years Basic Parent Training may be efficacious in reducing ADHD symptoms; however, these studies lack behavioral observations of ADHD symptoms, teacher ratings, and measures of functional impairment. PCIT is a parent training program designed for young children that may have features especially beneficial to young children with ADHD. Specifically, an intensive individualized treatment approach such as PCIT may prove useful for some families of children with ADHD (Chronis et al., 2004). Additionally, PCIT has been disseminated to many community settings throughout the United States and abroad and research suggests that it is cost effective compared to untreated and persistent disruptive behavior (i.e., Goldfine, Wagner, Branstetter, \& McNeil, 2008). Although this treatment has been studied extensively with children with DBDs and has included children with ADHD in research, the effects of PCIT on ADHD symptoms and associated impairment have been unclear. Therefore, the goal of the current study was to determine whether PCIT reduces ADHD symptoms and impairment. Given the neurological underpinnings of ADHD (Barkley, 1998), 
PCIT was not expected to -eure" ADHD. Instead, treatment was intended to give parents tools to manage their child's symptoms in particular situations (e.g., during mealtime behavior and when completing homework). Specifically, the current study employed a single subject nonconcurrent multiple baseline design to address the effects of PCIT on ADHD symptoms and impairment to test the following hypotheses:

\section{Hypotheses (Observable Behavior):}

1.) Child On-Task Behavior: The primary hypothesis was that the percentage of child on-task behavior would visibly increase during the CDI phase of the intervention and changes would maintain or increase during PDI and during the 2-month follow-up visit. The increase in on-task behavior was hypothesized based on the theoretical underpinnings of PCIT and unique components that theoretically target attention to task (e.g., Eyberg, 1998; Lemaster et al., 2010; Wagner \& McNeil, 2008). Child on-task behavior was assessed with an observational task utilizing the Revised Edition of the School Observational Coding System (REDSOCS; Jacobs et al., 2000) during a structured parent-child interaction.

2.) Child Out-of-Area Behavior: The second observable hypothesis was that the percentage of child out-of-area behavior would visibly decrease during the CDI phase of the intervention and changes would maintain or increase during PDI and during the 2-month follow-up visit. The hypothesized decrease in out-of-area behavior was based on theories underlying PCIT and specific components relevant to hyperactivity (Eyberg, 1998; Wagner \& McNeil, 2008). Out-ofarea behavior was observed during Child Directed Interactions as part of the Dyadic ParentChild Interaction Coding System Observations - 3rd Edition (DPICS-III; Eyberg et al., 2005). Exploratory Variables (Standardized Rating Scales): 
1.) Parental Report of ADHD Symptoms: Based on previous research (e.g., Eisenstadt et al., 1993; Schuhmann et al., 1998; Matos et al., 2006, 2009) it was expected that parental report of child ADHD symptoms would decrease from pretreatment to mid-treatment, post-treatment, and follow-up. Child ADHD symptoms were measured by parental report on the ADHD subscales of the Conners' Parent Rating Scale - Revised Short Version (CPRS-RS; Conners, 1997) and the DSM-IV Structured Interview for Disruptive Behavior Disorders (McNeil et al., 1991).

2.) Parent Report of Child Impairment: Based on skills targeted in PCIT (Wagner \& McNeil, 2008), it was expected that parents would report reductions in child impairment in different domains (relationship with playmates, relationship with brothers and sisters, relationship with parents, academic progress, self-esteem, family in general, overall impairment) at post-treatment and at follow-up in comparison to pretreatment levels of impairment. Child impairment was measured via parental report on the Impairment Rating Scale (IRS; Fabiano et al., 2006). 3.) Teacher Rating of Child Symptoms: It was expected that teachers would report reductions in child oppositional behavior but that they would not report differences in ADHD symptoms at post-treatment and follow-up in comparison to teacher ratings at pretreatment consistent with previous research on school generalization (McNeil et al., 1991). Teacher reports of child symptoms were measured using the Oppositional Subscale and ADHD Index of the Conners' Teacher Rating Scale- Revised Short Version (CTRS-RS; Conners, 1997).

4.) Parent Report of Child Disruptive Behavior: It was expected that parents would report reductions in the frequency of child disruptive behaviors (i.e., noncompliance, defiance, aggression) at post-treatment and at follow-up compared to pretreatment levels of disruptive behavior consistent with PCIT research (e.g., Schuhmann et al., 1998). Child disruptive behavior was assessed utilizing the Eyberg Child Behavior Inventory (ECBI; Eyberg \& Pincus, 
1999), the Oppositional Subscale of the Conners' Parent Rating Scale - Revised Short Version (CPRS-RS Conners, 1997) and the ODD and CD scales on the DSM-IV Structured Interview for Disruptive Behavior Disorders (McNeil et al., 1991).

5.) Parenting Stress: Based on PCIT research (e.g., Eisenstadt et al., 1993; Eyberg et al., 2001; Schuhmann et al., 1998), it was expected that parents would endorse less parenting stress at posttreatment and follow-up compared to pretreatment levels of stress. Parenting stress was measured based on self report on the Parenting Stress Index -Short Form (PSI-SF; Abidin, 1995) 6.) Consumer Satisfaction: It was expected that parental report at mid-treatment, post-treatment and at follow-up would indicate that this treatment is acceptable for parents of children with ADHD consistent with previous research (Matos et al. 2006, 2009). This was evaluated by comparing treatment acceptability ratings to average ratings in PCIT research. Treatment acceptability was assessed on the Therapy Attitude Inventory (TAI; Eyberg, 1974).

\section{Method}

\section{Participants}

Participants in the study included 4 mothers of a child ages 5-6 with ADHD. Motherchild dyads were recruited through ads given to daycares, preschools, head-starts, pediatricians, psychologists, psychiatrists, and other agencies throughout the community. Also, individuals who contacted the PCIT lab about other related studies and clinical services were provided with information about the project if they appeared eligible. Participants were eligible to participate when they were available and willing to invest the required time into completing the assessments and therapy, and when they met selection criteria. The specific selection criteria were as follows: (a) the child had a previous diagnosis of ADHD, (b) the diagnosis was confirmed based on parental report on a structured DSM-IV clinical interview (DSM-IV Structured Interview for 
Disruptive Behavior Disorders; McNeil et al., 1991), a score in the clinical range on ADHD Index of the CPRS-RS, and parental report of clinically-significant impairment in at least two specific domains on the IRS, (c) the child's percentage of on-task behavior was less than 50 during the baseline condition, and (d) the child was either not currently on medication for ADHD, or, if the child was taking medication, the family had to verbally express satisfaction with dosing (i.e., improvement in symptoms and minimal side effects) and state an intention to maintain the current dosing. Exclusion criteria included the presence of a diagnosis (based on parental report) that might better account for their ADHD symptoms including Mood Disorder NOS, Bipolar Disorder, Mental Retardation or a Pervasive Developmental Disorder (PDD) and if the multi-method pretreatment assessment did not support the ADHD diagnosis. Participants were compensated for their time completing assessment measures. In particular, they received the following amounts at evaluations: $\$ 50$ for pretreatment, $\$ 50$ for mid-treatment, $\$ 50$ for posttreatment, and $\$ 100$ for follow-up. No children evaluated were excluded from the current study as all children enrolled met criteria for treatment. Individual child, mother, and family characteristics are described below with names changed to protect family confidentiality.

Phillip was a 6-year-old white male who participated in the study with his 31-year-old biological mother. He attended kindergarten at a public elementary school. Phillip's parents were divorced, he resided with his mother, and he did not have any siblings. His biological father lived in another state and they had limited contact, although his father unexpectedly visited him when the family was in the CDI phase of the study. Phillip was referred through a therapist in a private practice. His mother had a bachelor's degree and was employed full time in a temporary position. Family income was less than $\$ 20,000$ based on maternal report. His mother also disclosed experiencing depression and anxiety. During the course of the study, she 
began psychotropic medication for anxiety prescribed by her primary care physician. His mother disclosed Phillip's father had a diagnosis of ADHD.

Phillip's medical history was significant for a past diagnosis of ADHD and he did not have a history of any other medical, intellectual, or mental health problems. He was first diagnosed with ADHD at age 4 by a psychologist in Florida, where he resided before his parents' divorce, and subsequently was prescribed medication by his pediatrician. A neuropsychologist also completed testing with results demonstrating performance consistent with an ADHD diagnosis. Past treatment attempts included behavioral interventions and medication. His mother reported he attended weekly sessions for 6 months with a therapist who met individually with Phillip for half of each session and worked with the family for the other half. Based on his mother's report, techniques used in this treatment mainly consisted of behavior charts and tangible rewards. Additionally, Phillip was taking a morning and mid-day dose of Dexedrine (5 mg). His medication and dosing had been consistent for 4 months before enrollment in the current study. His mother verbally expressed satisfaction with his medication (i.e., reduction in ADHD symptoms and absence of adverse effects) as well as an intention to keep the dose stable if possible during treatment. Also, sessions were scheduled at evening and weekend times to observe Phillip at consistent times when the amount of stimulant medication left in his bloodstream was anticipated to be less than the amount resulting in therapeutic effects. He previously took Adderall for ADHD in Florida; however, this medication was discontinued because he experienced tics.

Phillip's mother endorsed 7 symptoms of ODD, 5 symptoms of inattention, 9 symptoms of hyperactivity/impulsivity, and 1 symptom of CD on the DSM-IV Structured Interview for Disruptive Behavior Disorders at pretreatment. Symptoms of ADHD had been present 
continuously since age 4 per her report. Phillip's score on the ADHD Index of the CPRS-RS was clinically elevated $(T=65)$. His mother's ratings suggested clinically-significant impairment in his relationship with parent, academic progress, self-esteem, family functioning, and overall functioning. Phillip's teacher's rated him as having clinically elevated Oppositional Problems $(T=69)$ on the CTRS-RS and his ADHD subscales were within the normative range. During the pretreatment baseline, Phillip's on task behavior ranged from 16.67-23.33\% (5-7 intervals out of 30 ) with an average of $20 \%$ (6 intervals).

Matthew was a 6-year-old white male who participated in the study with his 24-year-old biological mother. He was referred from a therapist in private practice. Matthew attended first grade at a public elementary school. Matthew resided with his biological mother, did not have any siblings and did not have any contact with his biological father who lived out of state.

Highest grade completed by his mother was high school; however, she reported completing some prerequisite courses for college. His mother worked full-time for a credit union. Family income fell into the $\$ 20,000-\$ 40,000$ range per self report. Matthew's mother disclosed she previously was diagnosed with bipolar disorder and reported current symptoms of depression. She reported a paternal family history of ADHD.

Matthew's medical history was significant for past diagnoses of ADHD and ODD. He did not have any intellectual or medical problems. Psychiatric diagnoses were initially made by a psychologist in a community mental health center when Matthew was 5-years-old and his pediatrician began prescribing him stimulant medication at this time. Diagnoses were confirmed by a psychologist following a neuropsychological evaluation. Previously, Matthew's mother reported trying therapy and medications to manage Matthew's behavior. Specifically, Matthew attended approximately 10 sessions of 1-on-1 therapy at age 5 . Also, 3 months before enrolling 
in the study, Matthew and his mother attended biweekly sessions with a therapist in private practice. His mother stated these sessions mainly consisted of the therapist working 1-on-1 with Matthew. She was unable to recall the names of previous medications but expressed a belief that Matthew had been on the majority of medications with the exception of Vyvanse. At pretreatment, Matthew was taking $27 \mathrm{mg}$ of Concerta ER in the morning. Although his mother reported some dissatisfaction with his medication, she did not foresee any medication changes due to insurance restrictions. All assessment and treatment sessions occurred in the late afternoon and early evening to allow for consistent observations of Matthew at a time that the amount of stimulant medication remaining in his bloodstream was expected to be outside of the therapeutic range

At the pretreatment assessment, Matthew's mother rated 8 symptoms of ODD, 9 symptoms of inattention, 9 symptoms of hyperactivity/impulsivity, and 3 symptoms of CD as occurring pretty often" or *ery often" On the DSM-IV Structured Interview for Disruptive Behavior Disorders She reported noticing these symptoms at age 4. Prior to beginning treatment, Matthew's score on the ADHD Index of the CPRS-RS fell into the clinical range $(T=$ 76) and his scores on the IRS demonstrated clinically-significant impairment in his relationships with playmates, relationship with parent, academic progress, self-esteem, family functioning, and overall functioning. Teacher ratings were also consistent with a diagnosis of ADHD and the following CTRS-RS subscales fell into the clinical range: Oppositional Problems $(T=69)$, Hyperactivity $(T=71)$, and ADHD Index $(T=69)$. During baseline observations, Matthew's ontask behavior ranged from 43.33-46.67\% (13-14 intervals out of 30$)$ with a mean of $45.8 \%$ (13.75 intervals). 
Jacob was a 5-year-old white male who participated in the study with his 31-year-old biological mother. He resided with both his biological parents and his 2-year-old brother. His mother had an associate's degree and worked full-time. Family income ranged from $\$ 20,000$ $\$ 40,000$. Jacob attended kindergarten at a public elementary school. Both of his parents disclosed having diagnoses of ADHD. Jacob's mother heard about the study through word-ofmouth. He was assessed through a departmental psychology clinic by graduate students who were not affiliated with the study. Prior to enrollment, Jacob had never taken any psychotropic medication and his mother expressed a strong desire to pursue other treatment options. Jacob's medical and psychiatric history was unremarkable.

Jacob's mother endorsed 3 symptoms of ODD, 9 symptoms of inattention, 6 symptoms of hyperactivity/impulsivity, and 0 symptoms of CD on the DSM-IV Structured Interview for Disruptive Behavior Disorders during the initial pretreatment assessment. Based on her report, ADHD symptoms had been consistently present since age 3. At pretreatment, Jacob's score on the ADHD Index of the CPRS-RS fell into the clinical range $(T=84)$ and his mother's ratings on the IRS demonstrated clinically-significant impairment in his relationship with parents, academic progress at school, self esteem, and overall functioning. Based on teacher ratings, Jacob's scores on the following CTRS-RS also fell in the clinical range on Cognitive Problems/Inattention $(T=$ 90), Hyperactivity $(T=77)$, and ADHD Index $(T=80)$. On-task behavior ranged from 33.33$60 \%$ (10-18 intervals out of 30$)$ with a mean of $48 \%$ (14.4 intervals).

Charlie was a 5-year-old white male who participated in the study with his 34-year-old biological mother. He was referred by his outpatient individual therapist at a local psychiatric hospital. He attended kindergarten at a public elementary. Charlie resided with his biological 
parents and 11-year-old brother. His mother obtained an associate's degree and was not employed at the time of the study. Reported family income was between $\$ 20,000$ and $\$ 40,000$.

Charlie's medical history was significant for asthma and previous psychiatric diagnoses of ADHD, oppositional defiant disorder, obsessive compulsive disorder, and Tourette's Syndrome made by his treating psychiatrist. Additionally, a neuropsychologist previously assessed Charlie and documented findings consistent with an ADHD diagnosis. Psychiatric history included numerous psychotropic medications. Prior to enrollment in the study, Charlie had taken Adderall, Focalin, Depakote, Seroquel, Risperdal, and Abilify. At the time of enrollment, he took Ritalin, Zoloft, Clonidine, and Trazodone. Before the pretreatment assessment, Charlie's dose of stimulant medication had remained stable for 6 months. His mother expressed satisfaction with his current dose of stimulant medication and stated she did not foresee any changes in dosing. There were no reported changes in the dose of stimulant medication during the course of therapy. All assessment and treatment visits were scheduled in the late afternoon and early evening in order to consistently observe Charlie when the remaining stimulant medication in his bloodstream was likely lower than doses in the therapeutic range.

During the pretreatment assessment, Charlie's mother endorsed 3 symptoms of ODD, 6 symptoms of inattention, 7 symptoms of hyperactivity/impulsivity, and 0 symptoms of CD on the DSM-IV Structured Interview for Disruptive Behavior Disorders. Based on her report, ADHD symptoms had been present continuously since age 3. Additionally, at pretreatment Charlie's score on the ADHD Index of the CPRS-RS fell into the clinical range $(T=67)$ and his scores on the IRS demonstrated clinically-significant impairment in his relationship with playmates, relationship with brother, relationship with parents, academic progress, self-esteem, family functioning, and overall functioning. His teacher's ratings on the CTRS-RS placed him in 
the clinical range for Oppositional Problems $(T=72)$ and Cognitive Problems/Inattention $(T=$ 80). During baseline sessions, Charlie's on-task behavior ranged from 46.67-86.67\% (14-26 intervals out of 30$)$ with an average of $66.11 \%$ (19.83 intervals).

\section{Measures}

Conners' parent rating scale - revised short version. The Conners' Parent Rating Scale - Revised Short Version (CPRS-RS; Conners, 1997) is a 27-item scale on which parents report the extent of their child's externalizing symptoms by rating if a behavior is fot true at all," just a little true," pretty much true," or *ery much true." The CPRS-RS contains the following scales: Oppositional, Cognitive Problems, Hyperactivity, and an ADHD Index. Research on the scale has demonstrated adequate reliability and validity (Conners, 1997). Conners (1997) reported Cronbach's alphas on the scales ranging from .83 to .94 for preschoolaged children and test-retest reliability ranging from .62 to .85 . Additionally, the scales can discriminate between individuals with and without ADHD (Conners, 1997). In the current study, the ADHD Index was used to determine whether children met inclusion criteria and all scales were utilized to examine change. Mothers completed the CPRS-RS at each study visit as well as at each assessment point. Given the frequency of administration, parents were instructed to complete the items based on the child's behavior since the last appointment as opposed to the instructions where parents are told to rate their child's behavior during the past month.

Conners' teacher rating scale - revised short version. The Conners' Teacher Rating Scale - Revised Short Version (CTRS-RS; Conners, 1997) is a 28-item scale for teachers. On the scale, teachers rate the extent of the child's externalizing symptoms by indicating if a behavior is not true at all," just a little true," pretty much true," or *ery much true." The CTRS-RS contains the same scales as the CPRS-RS (Oppositional, Cognitive Problems, 
Hyperactivity, and an ADHD Index). Research on the teacher version of the scale has also demonstrated acceptable reliability and validity (Conners, 1997). Conners reported internal consistencies of individual scales ranging from .81 to .97 for preschool-aged children and 6-8 week test-retest reliability ranging from .72 to .92 for the teacher version. Additionally, the scales can discriminate between individuals with and without ADHD (Conners, 1997). In the current study, the CTRS-RS was completed whenever possible at the pretreatment, posttreatment, and follow-up assessments by the child's teacher or daycare provider. Although every attempt was made to get teacher ratings, the current study did not exclude children from participation if teacher ratings were not obtained (e.g., if the child was enrolled in the study over the summer and was not in school or if the participant was younger and had not begun formal schooling yet).

Demographics form. A demographics form was developed for use in the current study. This form included the following parent information: age, gender, marital status, occupation, education level, and ethnicity. The form also included questions on the following child information: age, gender, ethnicity, diagnoses received, date of received diagnoses/assessments, location/agency of diagnosis, types of services received currently or previously, and past and current medication status. Additionally, the form contained questions regarding the home environment (i.e., number and ages of siblings in the home and family income). In the current study, the demographics form was completed during the pretreatment assessment by the mother.

\section{DSM-IV structured interview for disruptive behavior disorders. The DSM-IV}

Structured Interview for Disruptive Behavior Disorders was developed to assist in diagnosing externalizing disorders in children (McNeil et al., 1991). Specifically, parents or caregivers are interviewed about the frequency and duration of DSM-IV symptoms of ADHD, ODD, and CD, 
and in order for the child to receive a diagnosis, parental report must meet DSM-IV criteria. During the interview, parents are asked to rate their child's behaviors (as compared to other same-age children) on a 4-point scale, indicating if the behavior occurs rarely, occasionally, pretty often, or very often and symptoms that occur pretty often or very often are considered present (Campbell, Ewing, Breaux, \& Szumowski, 1986). The interview has demonstrated high inter-observer agreement in research (i.e., Eyberg et al., 2001; McNeil et al., 1991; Schuhmann et al., 1998). In this study, the interview was conducted at pretreatment, mid-treatment, posttreatment, and follow-up assessments to monitor diagnostic status over the course of treatment.

Dyadic parent-child interaction coding system $-3^{\text {rd }}$ edition. The Dyadic Parent-Child Interaction Coding System $-3^{\text {rd }}$ Edition (DPICS-III; Eyberg et al., 2005) is a quantitative behavioral coding system for parent and child behaviors during laboratory structured social interactions (see Appendix A for definitions). During these observations, parents and children engage in three 5-minute tasks (CDI, PDI, and clean-up; see Appendix B for instructions). During CDI, the caregiver is instructed to let the child lead the play and to follow along with his or her play. During PDI, the parent is instructed to tell the child that it is the parent's turn to choose the activity and lead the play. Lastly, during clean-up, the parent instructs the child to pick up the toys in the play area. Research on the psychometrics (reliability and validity) suggests that the coding system has adequate properties (Eyberg et al., 2005). Eyberg and colleagues (2005) report inter-observer agreement ranging from kappas of .47 to .72 .

The primary purpose of the DPIC-III in the current study was to inform treatment progression. Formal DPICS-III observations were collected at pretreatment, mid-treatment, post-treatment, and follow-up. The following parental behaviors were coded: labeled praise, reflection, behavioral description, direct commands, indirect commands, questions, negative talk, 
child compliance, child noncompliance, and child no opportunity to comply (see Appendix C for coding sheet). Live clinical coding occurred at the start of each session to determine parental progress toward mastery. Specifically, during the CDI phase of treatment, the therapists observed the CDI interaction and during the PDI phase of treatment, the therapists observed both the CDI and PDI interactions.

During CDI observations, child out-of-area behavior was coded. Specifically, the intervals during which the child is out-of-the-area was recorded by using a 4.5 foot by 5.5 foot area carpet. Participants were given instructions to keep their whole body on the carpet during both observations and clinical coaching. If any part of the child's body was out of the area for any part of the 10 second interval, it was coded as out-of-area behavior.

Eyberg child behavior inventory. The Eyberg Child Behavior Inventory (ECBI; Eyberg \& Pincus, 1999) is a measure of disruptive behavior for children ages 2-16 completed by parents and/or caregivers. This 36-item measure yields two scores, an Intensity Score and a Problem Score. On the Intensity Scale, parents report the frequency of disruptive behaviors on a 7-point scale, ranging from 1 (never) to 7 (always) and on the Problem Scale, parents indicate whether or not the behavior is a problem for them by circling yes or no. Eyberg and Pincus (1999) reported internal consistency coefficients ranging from .93 to .95 and 10-month test-retest reliability coefficients of .75 for both the Intensity Score and the Problem Score. Additionally, the ECBI discriminates between clinic-referred children and children with disruptive behaviors and is sensitive to treatment effects (Eyberg \& Pincus, 1999). The clinical cutoff scores on the ECBI are 131 and 15 for the Intensity Score and Problem Score respectively (Eyberg \& Pincus, 1999). In accordance with procedures described in the PCIT manual (Eyberg, 1999), the primary 
caregiver completed the ECBI at each assessment point and at every therapy session (during both CDI and PDI).

Impairment rating scale. The Impairment Rating Scale (IRS: Fabiano et al., 2006) is a seven item scale on which parents report impairment on different domains necessary for an ADHD diagnosis based on DSM-IV criteria. On the form, parents rate the child's extent of impairment as well as the child's need for treatment in individual domains (relationship with playmates, relationship with brothers and sisters, relationship with parents, academic progress, self-esteem, family in general) and the overall impairment and need for treatment. Parental ratings are made by placing a mark along a continuum and a 7-point scale is utilized to score these ratings. Parental scores of three or more are indicative of clinically-significant impairment; and this rating discriminates between clinical and comparison children. Psychometric properties have been reported on a preschool sample (Fabiano et al., 2006). Specifically, 1-year stability correlations ranged from .54 to .76 , correlations with other measures of impairment are moderate to high (Fabiano et al., 2007). Additionally, the scale has demonstrated incremental validity when utilized concurrently with rating scales assessing symptoms of ADHD. The IRS was administered at pretreatment, mid-treatment, post-treatment, and follow-up assessments.

Parenting stress index -short form. The Parenting Stress Index-Short Form (PSI/SF; Abidin, 1995) is a measure designed to assess areas of stress experienced in the parenting role, including: parental distress, difficult child, and parent-child dysfunctional interaction. These three subscales are added together to form a total stress score. Parents rate the 36 -items on the PSI/SF using a 5-point likert scale ranging from strongly disagree to strongly agree. Test-retest reliability coefficients range from .68 to .74 and internal consistency coefficients range from .80 to .91 (Abidin, 1995). This version of the PSI is very highly correlated with the standard version 
and the norms are available for parents of children with ADHD. Parents completed the PSI-SF at each assessment visit (pre, mid, post, and follow-up).

\section{Revised edition of the school observation coding system (on-task behavioral ratings).}

A structured behavioral observation of percentage of on-task behavior was the primary dependent variable of the study and subsequently, was measured at every study visit as well as at the pretreatment, mid-treatment, post-treatment, and follow-up assessments. This task utilized a coding system modified from the Revised Edition of the School Observation Coding System (REDSOCS; Jacobs et al., 2000) that has previously been employed in pilot studies (Lemaster, et al., 2010; Tempel, 2009).

The behavioral observations consisted of a structured drawing situation involving the mother and the child. During the situation, raters assessed on-task behavior using an operational definition appropriate for the situation. Specifically, on-task behavior was defined as sustained attention on the activity such that a failure to look at or manipulate the materials (crayons and paper) constitutes off- task behavior. Additionally, participants were coded as on-task if they engaged in a conversation with their parent about the task; however, task irrelevant comments (i.e., off-topic comments and requests to end the task) were coded as off-task.

The task consisted of a 5-minute free-play in which the child was given instructions to draw with crayons (four different colors) and blank paper on the table. The parent was instructed to play with the child and use all of the parenting skills in his or her repertoire to keep the child on-task (i.e., before treatment the parent were able to use the techniques she knows, and during treatment the parent was able to use her newly acquired CDI and PDI skills).

Ratings of on- or off-task were made based on a modified version of the off-task behavior category on the REDSOCS (see Appendix D for record form). The off-task behavior category 
has demonstrated convergent validity with teacher ratings in classroom research (Jacobs et al., 2000). Therefore, in the absence of teacher ratings, these observations served as an objective measurement of the child's ADHD behaviors along with parental report. Raters familiar with the task and the operational definition of off-task behavior coded off-task behavior utilizing a 10second interval coding system. Specifically, when the child was not on-task at any point during the 10-seconds, the interval was coded as off-task. Raters were instructed to temporarily pause the coding if at anytime the interaction stopped (e.g., if the parent placed the child in timeout or the child needed to use the restroom). Raters then resumed timing and coding when the child physically returned to the task (regardless of whether the child's behavior was on- or off-task).

Therapy attitude inventory. The Therapy Attitude Inventory (TAI; Eyberg 1974) was developed to measure parental satisfaction with behavioral parent training programs at the completion of treatment and was revised by Eyberg to make the questions applicable for parents completing PCIT. Parents complete 10 questions rating different components of treatment on a 5-point likert scale. Components rated include change in child behavior problems, confidence in implementing intervention components, and general impressions of the treatment. Higher scores on the TAI are indicative of greater levels of parental satisfaction with treatment. Brestan, Jacobs, Rayfield, and Eyberg (1999) reported a Cronbach's alpha of .91 and test-retest reliability of .85. Parents completed the TAI in the proposed study during the mid-treatment, posttreatment, and follow-up assessments.

\section{Procedure}

Design considerations. The study utilized a single subject design due to practical constraints (i.e., time-limited dissertation research and limited access to clinical populations in a small city). Single subject designs can effectively rule out the same threats to internal validity as 
between-group designs through repeated measurement of the dependent variable and utilization of different baselines (Kazdin, 1982). Although single subject research is subject to limitations (i.e., questionable generalization of findings), one advantage of this design is that the repeated measurement of the dependent variable allows researchers to determine precisely when change occurs in treatment (Choate, Pincus, Eyberg, \& Barlow, 2005). For instance, Choate and colleagues (2005) used a single subject design to examine the effects of PCIT on separation anxiety and found greatest decreases in anxiety occurring during CDI. Given the criticism of multi-component treatments and the emphasis on detecting the active ingredients (i.e., Hoza et al., 2007), a single subject design may be advantageous to developing and testing hypotheses about active components of research, which is relevant in this study due to the hypothesis regarding change in on-task behavior during CDI.

Common single subject designs are the traditional ABA or reversal design and multiple baseline designs. Multiple baselines (across participants, settings, or behaviors) are often selected instead of sequential withdrawal or reversal designs in clinical research for practical or ethical reasons (Barlow \& Hershen, 1984). For instance, reversal is not feasible when a treatment results in long-lasting effects, such as PCIT's demonstrated improvement in the parentchild relationship and over-learning of parenting skills until they become habits." Although multiple baseline designs across participants are considered less rigorous in terms of experimental control, the nature of the treatment precludes the use of other baselines in PCIT (i.e., utilizing a multiple baseline across behaviors is not feasible because PCIT is a multicomponent treatment targeting many specific and global behaviors and interactions). Multiple baseline across participants designs have been successfully utilized in PCIT outcome research (i.e., Choate, et al., 2005; Ware, McNeil, Masse, \& Stevens, 2008). 
Study design. The current project employed a non-concurrent multiple baseline across participants, which is also occasionally referred to as a moving treatment design (Watson \& Workman, 1981). This design was primarily developed for use in applied settings in instances when the traditional multiple baseline design is not practical, ethical, or feasible (Watson \& Workman, 1981). This design is similar to a traditional multiple baseline design except the criterion that all participants must be enrolled and studied simultaneously is relaxed, and therefore, participants can start the study when they are recruited. Participants in this design are randomly assigned to a baseline that has been established a priori. This design controls for the same threats to internal validity as the traditional multiple baseline design. For instance, observing the same effect at three distinct points in time decreases the plausibility of history effects accounting for the change in the dependent variable (Watson \& Workman, 1981). Nonconcurrent multiple baseline designs across participants have been employed to experimentally examine the effects of a variety of interventions on behaviors with various populations, including the effects of a guided compliance procedure on noncompliance in typically developing preschoolers (Wilder \& Atwell, 2006); the effects of behavioral training on adherence to positive airway pressure in four preschoolers with Obstructed Sleep Apnea and developmental delays (Slifer et al., 2007); and a study evaluating the effects of PCIT on compliance in children with high-functioning autism (Masse, 2009). The current study utilized baselines of 3, 4, 5, and 6 . These lengths were selected to be feasible but also to provide some evidence to suggest that intervention effects were not the result of maturation (which was a concern given the target children's ages).

Additionally, in order to progress from baseline to CDI and from CDI to PDI, stability needed to be attained on measurement of on-task behavior. Specifically, stability criterion in the 
study was defined as three data points demonstrating a neutral or downward trend in percentage of child on-task behavior during baseline. This criterion was in addition to requirements for mastery of CDI and PDI which also was required before progressing treatment. Given that the percentage was computed from 30 10-second intervals, an increase or decrease of just one interval resulted in a percentage change of $3.33 \%$. Therefore, small changes in the baseline of up to $3.33 \%$ were defined as neutral.

Therapists. Three therapists, one primary therapist and two co-therapists administered PCIT. All therapists were graduate students from the Clinical Child Psychology Program at West Virginia University. The primary therapist had a Master's Degree and the co-therapists had at least a Bachelor's Degree in Psychology. Both therapists had clinical training and experience with PCIT and were provided with a manual (Eyberg, 1999) that outlines specific procedures for each treatment session. In addition, the primary therapist had a year of clinical research experience with PCIT utilizing a similar treatment design. Study therapists were supervised by a licensed clinical psychologist who is an expert in both conducting PCIT and supervising clinicians.

Treatment. Treatment adhered to the standard PCIT protocol (Eyberg, 1999) except that sessions occurred at the participants' homes as opposed to a clinic setting. Therapy was conducted twice a week in the home to minimize the research burden to the participants given the intensity and length of treatment. The standard PCIT protocol was modified slightly to accommodate this environment. Specifically, in clinic settings, therapists coach parents behind a one-way mirror utilizing bug-in-the-ear technology. However, this is not feasible in a home setting; therefore, therapists used in-room" coaching. This consisted of giving the child instructions to ignore the therapist's vocalizations and having the therapist sit close to the parent 
to provide feedback quietly. Although there has not been extensive research on this format, preliminary evidence suggests that this method is effective (Rayfield \& Sobel, 2000; Ware et al., 2008; Masse, 2009).

Study visits progressed from baseline to CDI to PDI. Specifically, after baseline criteria were met, the therapist conducted a CDI didactic to teach parents the CDI skills through instruction, modeling, and practice. The current study utilized a longer and more intensive CDI didactic than typically used in PCIT research and practice because the purpose of the study was to examine the effects of parental skills on child on-task behavior. In particular, it was expected that parental use of CDI skills would increase on-task behavior, yet if CDI started and the parents did not know any of the skills, the intervention would not measure the construct of interest. Therefore, it was expected that parents would start CDI coaching sessions with a higher skill level than typical parents. After parents demonstrated mastery of CDI skills and the child's ontask behavior was stable, a mid-treatment assessment was conducted. Following this assessment, PDI started. This phase of treatment also began with a didactic that explained and demonstrated the skills to parents and gave them a chance to practice. After the didactic, PDI coaching sessions commenced and continued until the parent reached mastery criteria and the child's ontask behavior was stable or increasing. Table 1 displays number of treatment sessions (didactic and coaching) in the current study and averages from other published investigations utilizing mastery criteria as opposed to a set number of sessions.

Following treatment mastery, the parent and child participated in a post-treatment assessment session that occurred within a week of mastery and in a follow-up assessment that occurred 10 to 13 weeks after the post-treatment assessment. The course of individual participant progress is described below. 
Phillip and his mother completed 3 baseline visits including the pretreatment assessment, 1 CDI didactic session, 7 CDI sessions, 1 PDI didactic, and 12 PDI coaching sessions. No family member or other individual was present at any study session. During PDI 7, clinicians worked with his mother on diaphragmatic breathing prior to coding and coaching due to her anxiety and distress over his escalating aggressive behavior. In particular, clinicians needed to implement a house rule for physical aggression consistent with family progress and PCIT protocol; however, his mother was experiencing visible physiological arousal and discomfort when implementing timeout. Given the anticipated increase in frequency of timeouts with the addition of this rule, clinicians felt that targeting relaxation was critical to safety in the home and intervention integrity (i.e., mother following through with timeout). Currently, relaxation is often targeted during PDI for families with parenting stress, anxiety, or who are at-risk for child abuse and neglect (McCoy, Funderburk, \& Chaffin, 2004). Phillip and his mother met criteria to finish treatment during PDI 10, which coincided with the last week of school. However, additional sessions were conducted because his on-task behavior was not stable or increasing at PDI 10. Phillip's mother's homework completion ranged from 2-6 practices for CDI with an average of 3.86 and 3-7 practices during PDI with an average of 5.

Matthew and his mother completed 4 baseline visits including the pretreatment assessment, 1 CDI didactic session, 5 CDI sessions, 1 PDI didactic, and 12 PDI coaching sessions. No family members or other individuals attended any treatment or assessment sessions. Although Matthew and his mother met criteria to finish treatment during the $10^{\text {th }}$ PDI session, his on-task behavior was not stable or increasing; therefore, additional sessions were scheduled. The decrease in on-task behavior coincided with the end of the school year. During treatment, Matthew's mother completed homework sheets between each session. According to 
her documentation, she practiced CDI skills 0-6 times a week outside of session and PDI skills 27 times. The only week she did not practice skills at all occurred between the CDI didactic and $1^{\text {st }}$ CDI session. Homework completion averaged 2.73 and 3.89 for CDI and PDI respectively. Jacob and his mother completed 5 baseline sessions including the pretreatment assessment, 1 CDI didactic session, 12 CDI coaching sessions, 1 PDI didactic session, and 8 PDI coaching sessions in addition to assessment visits. Although Jacob's mother met mastery criteria for CDI in 9 sessions, his on-task behavior did not meet the criteria necessary to change conditions. Progression through CDI was likely influenced by a 3-week vacation taken by the family between sessions 6 and 7. During CDI, clinicians worked with Jacob's mother at managing parenting stress beginning in CDI 4 where parental stress is first addressed with a handout. Given her self-disclosures regarding stress and a lack of enthusiasm during coaching, therapists encouraged her to schedule pleasant events. Additionally, due to parent and teacher concerns, therapists implemented a home-school note and grab bag reward program to facilitate parent-teacher communication and to target classroom disruptive behaviors (following directions, keeping hands and feet to himself, and working quietly). Jacob's home-school note began at mid-treatment and continued throughout PDI. Home-school notes have empirical support for reducing classroom concerns (e.g., Jurbergs, Palcic, \& Kelley, 2007) and have been used in PCIT (Verduin, Abikoff, \& Kurtz, 2008). As the timing of Jacob's enrollment in the study prevented clinicians from systematically evaluating generalization to school (i.e., different school year and teacher), clinicians felt it was important to provide consultation for school difficulties. During treatment, Jacob's mother completed homework regularly throughout the study except during the 3-week vacation because Jacob stayed with his grandmother during this 
time. Based on homework, Jacob and his mother practiced CDI skills 2-6 times a week with an average of 4 times. They practiced PDI skills 4-7 times a week with an average of 5.66.

In addition to assessment visits, Charlie and his mother completed 6 baseline sessions including the pretreatment assessment, 1 CDI didactic session, 7 CDI coaching sessions, 1 PDI didactic session, and 15 PDI coaching sessions. Other family members did not attend any coaching sessions; however, his father did attend the CDI didactic session. While his older brother and father were occasionally home during sessions, they remained in other areas of the home (bedroom with door closed). Although Charlie's mother met mastery criteria for CDI in 3 sessions, his on-task behavior did not meet the stability criterion, and therefore, the family completed 4 additional CDI sessions. He did not complete additional sessions of PDI following mastery. School consultation began following PDI 6 because Charlie was exhibiting noncompliant and aggressive behavior, prompting school officials to suspend him multiple times. He also was experiencing difficulty remaining seated and completing work. Despite attempts to consult with the school to develop a specific discipline plan at school in combination with classroom-wide contingency management, the school did not consistently implement recommendations. Subsequently, the family sought due process to address educational difficulties. Charlie's mother completed CDI homework 0-5 and PDI homework 3-6 times a week. Weeks with the lowest number of practices ( 0 and 1$)$ occurred over the winter holidays. Also, twice his mother did not fill out the homework sheet, which prompted clinicians to have her complete it in session per protocol (McNeil \& Hembree-Kigin, 2010). Homework completion averaged 3.11 during CDI and 4.14 during PDI.

Treatment integrity. Integrity was measured through the use of integrity checklists modified from the PCIT manual (Eyberg, 1999; see Appendix E for sample). Specifically, 
additional elements were be added to the checklist to account for specific components of the study (e.g., on-task behavioral ratings and administration of the CPRS-RS). Co-therapists completed independent integrity checklists for $27.19 \%$ of all sessions (assessment and treatment). Integrity rates were computed by adding the number of items addressed in session by the therapist by the total number of items and multiplying by 100 to obtain a percentage. Treatment integrity scores across sessions and participants averaged 98.33\%, ranging from 90$100 \%$. Missed session items, resulting in lower scores on integrity checks, included failing to review ECBI graphs with parents at the end of session, coaching less than 30 minutes due to disruptive child behavior, failing to have the parent review the rules for coaching, and not providing a specific detail regarding parent homework assignment.

Inter-observer agreement. All on-task ratings, out-of-area behavior, and DPICS-III ratings were coded live during therapy sessions and were videotaped during assessment sessions. A secondary observer coded $35.29 \%$ of CDI interactions to observe out-of-area behavior through live coding. Twenty five percent of on-task and out-of-area ratings during assessment sessions were coded by an observer who was unaware of the participant's treatment status.

A kappa value was calculated for each DPICS-III code used to determine treatment progression (i.e., Labeled Praise [LP], Behavioral Description [BD], Reflection [RF], Questions [QU], Indirect Command [IC], Direct Command [DC], Negative Talk [NT], Child Compliance [CCO], Child Noncompliance [NCO], and No Opportunity to Comply [NOC]). Calculated kappas were averaged across participants and sessions. The obtained averages and ranges were as follows: $\mathrm{LP}(M=.84$, range $=.80-.88), \mathrm{BD}(M=.80$, range $=.77-.86), \mathrm{RF}(M=.80$, range $=.75-.82), \mathrm{QU}(M=.85$, range $=.79-.95), \mathrm{IC}(M=.90$, range $=.86-.96), \mathrm{DC}(M=.91$, range $=.82-1.00), \mathrm{NT}(M=.96$, range $=.91-1.00), \mathrm{CCO}(M=.96$, range $=.90-1.00), \mathrm{NCO}(M=$ 
.96 , range $=.93-1.00)$, and NOC $(M=.90$, range $=.87-.96)$.

Kappa values were computed for each of these observations and averaged across participants and sessions. Average kappa for ratings of out-of-area behavior during live coding was 0.90 with a range of $0.89-0.94$. Inter-observer agreement across these videotaped assessment sessions by an observer who was unaware of treatment condition averaged 0.95 with a range of $0.78-1.00$.

Kappa values were computed for each live observation of on-task behavior was computed and averaged across participants and participants. The average kappa was 0.82 with a range of 0.80-0.87. Agreement across videotaped assessment observations with a coder unaware to treatment condition averaged 0.83 with a range of $0.76-0.90$.

\section{Results}

\section{On-Task Behavior}

Figure 1 demonstrates intervals of on-task behavior during coloring for Phillip, Matthew, Jacob, and Charlie across treatment phases and assessment sessions.

Phillip exhibited the lowest on-task behavior at pretreatment and across baseline sessions with on-task intervals ranging from 16.67-23.33\% (5-7 intervals out of 30). During these sessions, his on-task behavior averaged 20\% (6 intervals). Phillip's on-task behavior visibly increased during CDI and ranged from 50-76.67\% (15-23 intervals) with an average of 67.62\% (20.29 intervals) during this time. His on-task behavior was more variable in CDI ( $S D=3.04)$ compared to baseline $(S D=1.00)$. During the mid-treatment assessment, Phillip exhibited $60 \%$ on-task behavior (18 out of 30 intervals). Throughout PDI, Phillip's on-task behavior ranged from $36.67-90 \%$ (11-27 intervals). On-task behavior averaged 76.36\% (22.90 intervals) during this condition. Additionally, his on-task behavior was more variable during PDI ( $S D=4.80)$ 
compared to CDI. During the post-treatment and follow-up visits, Phillip's on-task behavior was $86.67 \%$ ( 26 intervals) and $83.33 \%$ ( 25 intervals) respectively. No data points during the pretreatment baseline overlapped with data points during CDI, PDI, or assessment visits (midtreatment, post-treatment, and follow-up).

Prior to CDI, Matthew's on-task behavior ranged from 43.33-46.67\% (13-14 intervals out of 30). His behavior was less variable than the other participants' on-task behavior prior to beginning treatment $(\mathrm{M}=45.83 \%$ or 13.75 intervals, $\mathrm{SD}=0.5)$. During CDI, Matthew's on-task behavior increased to $73.33-80 \%$ (22-24 intervals) with a mean of $76.67 \%$ ( 23 intervals). Variability of on-task behavior during CDI $(S D=0.71)$ was similar to pretreatment. His on-task behavior was $83.33 \%$ (25 intervals) during the mid-treatment assessment. Matthew's on-task behavior ranged from 50-93.33\% (15-28 intervals) during PDI. During this condition, mean ontask behavior was $76.36 \%$ (22.91 intervals). Additionally, his on-task behavior during PDI was more variable $(S D=4.28)$ compared to other phases. Matthew exhibited $86.67 \%$ (26 intervals) on-task behavior at both post-treatment and follow-up. During CDI, PDI, and assessment visits (mid-treatment, post-treatment, and follow-up), no data points overlapped with the pretreatment baseline.

Jacob's on-task behavior at pretreatment and across baseline sessions ranged from 33.33$60 \%$ (10-18 intervals out of 30$)$ with a mean of $48 \%$ (14.4 intervals). During CDI, his on-task behavior visibly was more variable $(S D=7.74)$ compared to his behavior at pretreatment $(S D=$ 2.97). His on-task behavior ranged from $6.67-90 \%$ (2-27 intervals) with a mean of $44.17 \%$ (13.25 intervals). At the mid-treatment assessment, he exhibited $46.67 \%$ on-task behavior (14 intervals). Throughout PDI, Jacob's on-task behavior increased and variability decreased from CDI observations $(S D=5.21)$. On-task behavior ranged from $53.33-93.33 \%(16-28$ intervals $)$ 
and averaged $79.52 \%$ (23.86 intervals). His on-task behavior was 76.67\% (23 intervals) and $80 \%$ (24 intervals) at post-treatment and follow-up respectively.

At pretreatment and during baseline sessions, Charlie's on-task behavior ranged from 46.67-86.67\% (14-26 intervals out of 30). On-task behavior averaged $66.11 \%$ (19.83 intervals). During CDI, Charlie's on-task behavior increased slightly and ranged from 40-93.33\% (12-28 intervals) with a mean of $77.62 \%$ (23.29 intervals). Variability visibly increased during CDI (SD $=5.99)$ as compared to baseline $(S D=4.45)$. At the mid-treatment assessment, his on-task behavior was $83.33 \%$ ( 25 intervals). His on-task behavior increased slightly during PDI and ranged from $60-90 \%$ (18-27 intervals) with a mean $80.89 \%$ (24.27 intervals). On-task behavior was $83.33 \%$ (25 intervals) at post-treatment and $86.67 \%$ (26 intervals) at follow-up.

\section{Out-of-Area Behavior}

Figure 2 demonstrates percentage of out-of-area behavior during CDI observations for Phillip, Matthew, Jacob, and Charlie across treatment phases and assessment sessions.

Phillip's out-of-area behavior during pretreatment and across baseline sessions ranged from $0-70 \%$ (0-21 intervals out of 30). Phillip's out-of-area behavior averaged 25.56\% (7.67 intervals). During CDI, Phillip's out-of-area behavior continued to vary with a range of 0$43.33 \%$ ( $0-13$ intervals) and an average of $11.90 \%$ (3.57 intervals). His out-of-area behavior was less variable during CDI $(S D=6.71)$ compared to the pretreatment baseline $(S D=11.59)$. Three data points (out of 7 total points) during CDI (CDI 1, 23.33\%, CDI 2, 13.33\%, and CDI 7 , 43.33\%) were higher than every baseline data point aside from the initial pretreatment session (70\%). At mid-treatment, Phillip's out-of-area behavior was 0\% (0 intervals). During PDI, Phillip continued to exhibit a low frequency of out-of-area behavior ranging from $0-10 \%(0-3$ intervals). Phillip did not exhibit any out-of-area behavior (0\%) during 7 of 12 PDI sessions. 
His mean out-of-area behavior was $2.42 \%$ (0.73 intervals). Variability was lower during PDI $(S D=1.10)$ compared to both the pretreatment baseline and CDI. Out-of-area behavior was $6.67 \%$ ( 2 intervals) and $0 \%$ (0 intervals) at post-treatment and follow-up respectively.

During the pretreatment baseline, Matthew's out-of-area behavior ranged from $0-46.67 \%$ (0-14 intervals out of 30). His baseline out-of-area behavior averaged 20\% (6 intervals). During CDI, Matthew's out-of-area visibly decreased, ranging from 0-6.67\% (0-2 intervals). His out-ofarea behavior averaged 2.67\% (0.8 intervals). Out-of-area behavior was less variable during CDI $(S D=1.10)$ compared to the pretreatment baseline $(S D=5.89)$. At mid-treatment, Matthew did not exhibit any out-of-area behavior $(0 \%)$. Out-of-area behavior continued to occur at a low frequency during PDI ( $0 \%$ out-of-area behavior during 8 of 11 coded PDI sessions). His out-ofarea behavior ranged from $0-16.67 \%$ with an average of $3.33 \%$ ( 1 interval). Out-of-area behavior was more variable in PDI $(S D=2.11)$ compared to CDI. Matthew's out-of-area behavior was $6.67 \%$ (2 intervals) at post-treatment and $0 \%$ (0 intervals) at follow-up.

Similar to Phillip and Matthew, Jacob's out-of-area behavior at baseline was variable, ranging from $0-36.67 \%$ (0-11 intervals out of 30). Prior to CDI, his out-of-area behavior averaged 20\% (6 intervals). Out-of-area behavior did not visibly decrease beginning with the $1^{\text {st }}$ CDI session. During CDI, Jacob's behavior continued to vary; however, the frequency of out-ofarea behavior decreased ( $0 \%$ during 6 out of 12 CDI sessions). Out-of-area behavior ranged from 0-36.67\% (0-30 intervals) with an average of $9.72 \%$ (2.92 intervals). Variability in out-of-area behavior was similar in CDI $(S D=4.01)$ compared to the pretreatment baseline $(S D=4.64)$. His out-of-area behavior was $0 \%$ at the mid-treatment assessment. Jacob did not exhibit any out-ofarea behavior during 6 out of 7 PDI sessions. His out-of-area behavior averaged $1.90 \%(0.57$ intervals). Jacob's out-of-area behavior was less variable during PDI ( $S D=1.51)$ compared to 
other phases. During both the post-treatment and follow-up assessments, Jacob did not exhibit any out-of-area behavior $(0 \%)$.

Charlie exhibited out-of-area behavior throughout the pretreatment baseline. During baseline, out-of-area behavior ranged from 13.33-76.67\% (4-23 intervals out of 30$)$. His out-ofarea behavior averaged 33.89\% (10.17 intervals). Charlie's out-of-area behavior visibly decreased when CDI began; however, his behavior remained variable $(S D=7.23$ during CDI compared to $S D=7.25$ during the pretreatment baseline), ranging from $0-60 \%(0-18$ intervals). Out-of-area behavior averaged 25.24\% (7.57 intervals) during this phase. Charlie did not exhibit any out-of-area behavior at mid-treatment (0\%). During PDI, Charlie's out-of-area behavior continued to fluctuate $(S D=7.47)$ and did not visibly decrease further. Charlie's out-of-area behavior ranged from $0-83.33 \%$ ( $0-25$ intervals) with an average of $25.78 \%$ ( 7.73 intervals). Charlie's out-of-area behavior was higher at post-treatment (46.67\%) compared to his last 4 PDI sessions. He exhibited less out-of-area behavior during the follow-up assessment (13.33\%).

\section{Parent Report of ADHD Symptoms}

Conners' parent rating scale - revised short version (CPRS-RS). CPRS-RS data for ADHD subscales across treatment phases are displayed graphically in Figure 3. At pretreatment, Phillip's scores on Hyperactivity $(T$-score $=85)$ and ADHD Index $(T$-score $=65)$ were clinicallyelevated. His score on Hyperactivity $(T$-score $=69)$ remained elevated at mid-treatment, but all other scales were within the normal range $(T$ 's $<65)$. All scales remained below the clinical cutoff at post-treatment and follow-up.

Matthew's scores on all scales were clinically-elevated at both pretreatment and midtreatment $(T$ 's $>65)$. All scores decreased and were no longer clinically-elevated at posttreatment and follow-up $(T$ 's $<65)$. 
Jacob's scores on all scales were clinically elevated at pretreatment $(T$ 's $>65)$. His Hyperactivity Score decreased below the clinical cutoff from pretreatment $(T$-score $=67)$ to midtreatment $(T$-score $=58)$. These scores remained below the clinical cutoff at both post-treatment and follow-up. By post-treatment and follow-up, Jacob's scores on Cognitive Problems/Inattention and ADHD Index fell below the clinical cutoff $(T$ 's $<65)$.

Charlie's scores on Cognitive Problems/Inattention $(T$-score $=68)$ and the ADHD Index $(T$-score $=67)$ were clinically-elevated at pretreatment. His score on ADHD Index fell to below the clinical cut-off at mid-treatment $(T$-score $=62)$. By post-treatment, Charlie's score on Cognitive Problems/Inattention fell within the normal range $(T$-score $=55)$. All of Charlie's scores fell or remained below the clinical cutoff at post-treatment and follow-up $(T$ 's $<65)$.

DSM-IV structured interview for disruptive behavior disorders. Participant data from the DSM-IV Structured Interview for Disruptive Behavior Disorders Rating Scale (DSMIV DBD) are displayed graphically in Figure 4. At pretreatment, maternal ratings placed Phillip above the symptom count needed for ADHD Predominantly Hyperactive/Impulsive Type (5 symptoms of Inattention and 9 symptoms of Hyperactivity/Impulsivity). The frequency of ADHD symptoms decreased slightly between pretreatment and mid-treatment (3 symptoms of Inattention and 8 symptoms of Hyperactivity/Impulsivity). Symptoms of Hyperactivity/ Impulsivity were still clinically-elevated at post-treatment (6 symptoms endorsed). His mother also endorsed 4 symptoms of Inattention at post-treatment. At follow-up, Phillip's mother's ratings placed him below the symptom count for ADHD (0 symptoms of Inattention, and 2 symptoms of Hyperactivity/Impulsivity).

Matthew's mother's ratings of symptoms were above the threshold for ADHD Combined Type (9 symptoms of Inattention and 9 symptoms of Hyperactivity/Impulsivity endorsed). 
These symptoms remained elevated at mid-treatment ( 8 symptoms of Inattention and 8 symptoms of Hyperactivity/Impulsivity endorsed). By follow-up, his symptoms of Inattention were below the clinical cutoff ( 3 symptoms endorsed). He continued to exhibit clinicallysignificant symptoms of Hyperactivity/Impulsivity (7 symptoms endorsed). At follow-up, all ADHD symptoms were below diagnostic threshold (4 symptoms of Inattention, and 5 symptoms of Hyperactivity/Impulsivity).

Jacob met symptom criteria for ADHD combined type based on maternal ratings at pretreatment ( 9 symptoms of Inattention and 6 symptoms of Hyperactivity/Impulsivity). Ratings of ADHD symptoms remained elevated at mid-treatment (8 symptoms of Inattention and 6 symptoms of Hyperactivity/Impulsivity). At post-treatment, Jacob's mother did not endorse any symptoms of ADHD Inattention or Hyperactivity/Impulsivity. She rated 2 symptoms of Hyperactivity/Impulsivity as present at follow-up.

Charlie's mother rated him above the symptom threshold for ADHD Combined Type (6 symptoms of Inattention and 7 symptoms of Hyperactivity/Impulsivity. At mid-treatment her ratings placed Charlie above threshold for Inattention ( 8 symptoms endorsed) and below the threshold for Hyperactivity/Impulsivity (2 symptoms endorsed). By post-treatment, endorsed symptoms were below the threshold ( 3 symptoms of Inattention and 2 symptoms of Hyperactivity/Impulsivity). By follow-up Inattention symptoms were above threshold (6 symptoms endorsed). He also exhibited more symptoms of Hyperactivity/Impulsivity at followup (4 symptoms endorsed)

\section{Parental Ratings of Impairment}

Impairment Rating Scale (IRS) scores are displayed graphically in Figure 5. Phillip and Matthew's parents did not rate impairment with siblings because both participants were only 
children. At pretreatment, Phillip's mother rated his impairment as clinically-significant on the following domains: Relationship with Parent (6), Academic Progress (3), Self Esteem (3), Family Functioning (6), and Overall (Global Impairment) (4). Her rating of his impairment in Relationships with Peers (1) was below the cutoff for clinically-significant impairment. By midtreatment, these scores decreased but the majority remained in the clinically-significant range: Relationship with Parent (3), Self Esteem (3), Family Functioning (3), and Overall Impairment (4). Impairment in Academic Progress was below the threshold of clinical-significance by midtreatment (2) and impairment in Relationships with Peers remained stable. Impairment was further reduced by post-treatment with all scores below the cutoff of clinical-significance ( 1 on all domains aside from Relationships with Peers which increased from 1 to 2). By follow-up, several ratings of impairment increased but remained below the clinical cutoff: Relationship with Parent (2), Academic Progress (2), Self Esteem (2), and Overall Impairment (2). All other ratings were below clinical-significance (1) at follow-up.

Matthew's impairment was clinically-significant in all domains based on maternal ratings at pretreatment. She rated him as maximally impaired, scores of 6, on the following domains: Relationship with Parent, Self Esteem, and Overall (Global Impairment). All other domains were rated as 5's (Relationships with Peers, Academic Progress, and Family Functioning). During the mid-treatment assessment, all scores remained elevated above the clinical cutoff. Ratings of Impairment in the following domains decreased by one point: Relationship with Parent, Self Esteem, and Relationships with Peers. Impairment ratings in the domains of Academic Progress and Family Functioning increased by one point and Overall Impairment remained stable. At post-treatment, Matthew's impairment in Relationships with Peers (2) and Relationship with Parent (1) were no longer clinically-significant. Other scores decreased but 
remained above the clinically-significant threshold: Academic Progress (4), Self Esteem (4), Family Functioning (3), and Overall Impairment (3). The majority of maternal ratings at followup were below the clinical cutoff. Specifically, impairment ratings in Relationships with Peers (1), Relationship with Parent (0), Self Esteem (0), and Overall Impairment (2) were below the threshold for clinical significance. Ratings of Impairment in Family Functioning (5) and Academic Progress (4) were still elevated at follow-up.

Jacob's mother's ratings indicated clinically-significant impairment in all domains aside from Relationship with Sibling (2) at pretreatment. Specifically, he obtained ratings of 3 in Relationships with Peers and Family Functioning, a rating of 4 in Self Esteem, ratings of 5 in Relationship with Parents and Overall Impairment, and a rating of 6 in Academic Progress. At mid-treatment maternal ratings demonstrated impairment in all domains. Impairment ratings in Academic Progress and Relationship with Parents remained stable. Ratings suggested increased impairment in Relationships with Peers increasing from 3 to 5, Self Esteem increasing from 4 to 6, Family Functioning, increasing from 3 to 4, Overall Impairment increasing from 5 to 6 . Also, impairment in Relationship with Siblings increased from below the clinical cutoff to above increasing from 2 to 4 . Maternal ratings decreased to 0 , the lowest possible rating, on all domains at post-treatment and remained stable at follow-up.

At pretreatment, Charlie's mother's ratings placed him above the clinical cutoff on all domains with a score of 5 on Relationships with Peers and scores of 6 on all other domains (Relationship with Sibling, Relationship with Parents, Academic Progress, Self Esteem, Family Functioning, and Global Impairment). At mid-treatment, ratings on impairment decreased from 6 to 4 for all domains except Relationships with Peers which remained stable at 5 . By posttreatment, maternal ratings of impairment indicated decreased impairment in most domains with 
scores below the clinical threshold on Relationship with Sibling (1), Relationship with Parents (0), and Family Functioning (1). Impairment ratings of 4 in Relationships with Peers, 5 in Academic Progress, 3 in Self Esteem, and 3 in Overall Impairment remained clinicallysignificant. Additionally, at follow-up, all scores were above the clinical cutoff: Relationships with Peers (6), Relationship with Sibling (3), Relationship with Parents (3), Academic Progress (6), Self Esteem (5), Family Functioning (5), and Overall Impairment (5).

\section{Teacher Ratings}

Teacher ratings on the CTRS-RS were not obtained at post-treatment and follow-up because Charlie, Phillip, and Matthew finished treatment during the summer. Ratings were not obtained for Jacob because he had a different teacher at post-treatment than at pretreatment. Therefore, the hypothesis that reductions in ODD symptoms would generalize to the school setting and that ADHD symptoms would remain stable could not be empirically examined on a standardized teacher rating scale.

\section{Parent Report of DBD Symptoms}

Eyberg child behavior inventory (ECBI). ECBI results are presented graphically in Figure 6. At pretreatment, Phillip's mother rated him in the clinical range ( $T$-score $\geq 60)$ on both the Intensity and Problem Scale ( $T$-scores $=73$ and 76, respectively). Both scores were within the normal range at mid-treatment (Intensity $T$-score $=48$ and Problem $T$-score $=55$ ). These scores decreased further at post-treatment (Intensity $T$-score $=48$ and Problem $T$-score $=51)$ and follow-up (Intensity $T$-score $=38$ and Problem $T$-score $=41$ ).

Matthew's scores on the ECBI were above the clinical cutoff at both pretreatment (Intensity $T$-score $=79$ and Problem $T$-score $=78)$ and mid-treatment (Intensity $T$-score $=81$ and Problem $T$-score $=81$ ). All scores decreased to within normal range at post-treatment (Intensity 
$T$-score $=43$ and Problem $T$-score $=51)$ and follow-up (Intensity $T$-score $=49$ and Problem $T$ score $=55)$.

Jacob's scores were also clinically-significant at both pretreatment and mid-treatment. In contrast to Matthew, his scores decreased between pretreatment (Intensity $T$-score $=75$ and Problem $T$-score $=76$ ) and mid-treatment (Intensity $T$-score $=68$ and Problem $T$-score $=62$ ) . His scores were within the normal range at post-treatment (Intensity $T$-score $=36$ and Problem $T$-score $=41)$ and remained stable at follow-up (Intensity $T$-score $=36$ and Problem $T$-score $=$ 41).

Charlie's scores on the ECBI were clinically-significant at pretreatment (Intensity $T$-score $=71$ and Problem $T$-score $=75$ ). By mid-treatment, scores had decreased and his Intensity $T$ score of 59 was below the clinical cutoff; however, his Problem $T$-score remained clinically elevated $(T$-score $=69)$. At post-treatment, Charlie's Intensity $(T$-score $=44)$ and Problem Scales $(T$-score $=49)$ were within normal range. However, scores increased between posttreatment and follow-up. His Problem Scale $(T$-score $=65)$ fell into the clinical range at followup and his Intensity Scale $(T$-score $=54)$ remained in the normal range.

Table 2 displays raw scores at assessment points on the ECBI Intensity and Problem Scales in the current study in comparison to PCIT randomized controlled trials and in-home studies. Overall, ECBI scores on both Scales were higher at pretreatment and decreased more in comparison to other empirical investigations. In comparison to results obtained at mid-treatment by Eisenstadt and colleagues (1993), current raw scores were similar on the Intensity Scale (mean of 146.75 and standard deviation of 49.82) compared with families randomly assigned to CDI first (Intensity mean of 143.2 and standard deviation of 24.0). Scores from Matos et al (2009) were not included in the table because $T$-scores were presented instead of raw scores. In 
comparison to Matos and colleagues, the ECBI Intensity mean was higher at pretreatment in the current study, Intensity mean of 74.5 and standard deviation of 1.26 and Problem mean of 76.25 and standard deviation of 1.26, than the PCIT group (Intensity mean of 68.86 and standard deviation of 8.46 and Problem mean of 77.22 and standard deviation of 8.26), and the wait-list control group (Intensity mean of 71.93 and standard deviation of 9.79 and Problem mean of 78.98 and standard deviation of 6.46). Both Intensity and Problem Scores were lower at posttreatment in the current study (Intensity mean of 42.75 and standard deviation of 4.94 and Problem mean of 48.00 and standard deviation of 4.76) than the PCIT group (Intensity mean of 51.52 and standard deviation of 10.51 and Problem mean of 56.25 and standard deviation of 11.94 ) and the wait-list control group (Intensity mean of 68.36 and standard deviation of 9.74 and Problem mean of 77.36 and standard deviation of 6.78). Scores remained lower on both scales in the current study at follow-up (Intensity mean of 44.25 and standard deviation of 8.66 and Problem mean of 50.50 and standard deviation of 11.70) compared to the PCIT group (Intensity mean of 54.07 and standard deviation of 8.38 and Problem mean of 54.24 and standard deviation of 11.66).

Conners' parent rating scale - revised short version (CPRS-RS). CPRS-RS data across treatment phases are displayed graphically in Figure 3. At pretreatment, Phillip's scores on the Oppositional Subscale $(T$-score $=77)$ was clinically elevated but decreased to within the normal range at mid-treatment $(T$-score $=59)$. This score remained in the normal range at posttreatment $(T$-score $=48)$ and follow-up $(T$-score $=42)$.

Matthew's score on the Oppositional Subscale was clinically-elevated at both pretreatment $(T$-score $=85)$ and mid-treatment $(T$-score $=85)$. This score decreased and was no longer clinically-elevated at post-treatment $(T$-score $=45)$ and follow-up $(T$-score $=52)$. 
Jacob's Oppositional Subscale was clinically elevated at pretreatment $(T$-score $=68)$. This score decreased to below the clinical cut-off $(T$-score $=57)$ by mid-treatment. His score remained below the clinical cutoff at both post-treatment and follow-up $(T$-scores $=39)$. Charlie's score on the Oppositional Subscale was below the clinical cutoff at pretreatment ( $T$ scores $=62)$. His score on this Subscale remained below the clinical cutoff at mid-treatment $(T-$ scores $=54)$, post-treatment $(T$-score $=40)$, and follow-up $(T$-score $=54)$.

DSM-IV structured interview for disruptive behavior disorders. Participant data from the ODD and CD scales of the DSM-IV Structured Interview for Disruptive Behavior Disorders Rating Scale (DSM-IV DBD) are displayed graphically in Figure 4. At pretreatment, Phillip exhibited 7 symptoms of ODD, placing him above the symptom cutoff for ODD based on parental report. The frequency of ODD symptoms decreased over the course of treatment to 4 symptoms at post-treatment, indicating he still experienced clinically-elevated symptoms. At follow-up, Phillip's mother's ratings placed him below the symptom count (1 symptom of ODD). She endorsed 1 symptom of CD as present at pretreatment and post-treatment. She did not endorse any CD symptoms at mid-treatment or follow-up.

Matthew's mother's ratings of ODD and CD placed him above the symptom threshold for diagnoses of ODD (8 symptoms endorsed) and CD (3 CD symptoms endorsed). These symptoms decreased over the course of treatment. By mid-treatment, Matthew did not exhibit any symptoms of $\mathrm{CD}$, based on maternal rating and by post-treatment, maternal ratings of ODD (1 symptoms endorsed) were within the normal range. At follow-up, these symptoms were below diagnostic threshold (2 ODD symptoms and $0 \mathrm{CD}$ symptoms).

Jacob's mother rated 3 symptoms of ODD as present at pretreatment. Based on her ratings at mid-treatment, Jacob's 4 symptoms of ODD were above the threshold. At post- 
treatment and follow-up, Jacob's mother did not endorse any symptoms of ODD. She did not endorse any symptoms of CD across any of the assessment visits.

Charlie's mother rated 3 symptoms of ODD as present at pretreatment. By posttreatment, she did not endorse any ODD symptoms. Based on her report, 2 symptoms of ODD were present at follow-up. Across study visits, Charlie's mother did not endorse any symptoms of CD.

\section{Parenting Stress}

Parenting Stress Index - Short Form (PSI-SF) results are presented in Figure 7. The majority of Phillip's scores fell within the normal range $\left(<85^{\text {th }}\right.$ percentile $)$ across treatment phases with the exception of Parent-Child Dysfunctional Interaction which fell into the clinical range at pretreatment $\left(90^{\text {th }}\right.$ percentile $)$.

Matthew's mother's ratings of parenting stress on all domains were within the clinical range at pretreatment and mid-treatment $\left(>85^{\text {th }}\right.$ percentile $)$. At post-treatment, her score was within the normal range on Difficult Child $\left(55^{\text {th }}\right.$ percentile); however, this score was clinicallyelevated at follow-up $\left(85^{\text {th }}\right.$ percentile). Additionally, her score on Parent-Child Dysfunctional Interaction decreased between post-treatment $\left(90^{\text {th }}\right.$ percentile $)$ and follow-up $\left(70^{\text {th }}\right.$ percentile). Parental Distress and Total Stress remained clinically-elevated across treatment phases $\left(>85^{\text {th }}\right.$ percentile).

The majority of Jacob's mother's scores fell within the normal range across treatment phases $\left(<85^{\text {th }}\right.$ percentile). Her scores on the Difficult Child were elevated at pretreatment and mid-treatment $\left(90^{\text {th }}\right.$ percentile at both phases) and decreased to within the normal range at posttreatment and follow-up ( $1^{\text {st }}$ percentile at both phases $)$. 
All of Charlie's scores were clinically elevated at pretreatment and mid-treatment $\left(>85^{\text {th }}\right.$ percentile). Parental Distress decreased from clinically-significant $\left(95^{\text {th }}\right.$ percentile at pretreatment) to within the normal range at post-treatment and follow-up $\left(80^{\text {th }}\right.$ percentile at both phases). Additionally, Parent-Child Dysfunctional Interaction decreased from clinicallysignificant at pretreatment $\left(90^{\text {th }}\right.$ percentile) to below the clinical cutoff at post-treatment $\left(75^{\text {th }}\right.$ percentile). However, this score was above the clinical-cutoff at follow-up ( $90^{\text {th }}$ percentile).

\section{Consumer Satisfaction}

Phillip's mother's Treatment Attitude Inventory (TAI) score was lowest at mid-treatment, 40 (out of 50), in comparison to a post-treatment score of 47 . In comparison, her rating of satisfaction was highest at follow-up, 49.

At mid-treatment, Matthew's mother rated treatment acceptability as a 41. She reported increased consumer satisfaction at post-treatment, 44; however, her rating was lower at followup, 39.

Jacob's mother's TAI score was 42 at mid-treatment. At both post-treatment and followup, her rating indicated maximal satisfaction with treatment (i.e., scores of 50 at both assessments).

At mid-treatment, Charlie's mother's score of 38 on the TAI was lower than all other participants. She reported maximal satisfaction with treatment at post-treatment (50) and reported a score of 48 on the measure at follow-up.

Table 3 displays mean scores of consumer satisfaction at each phase of treatment in comparison to other empirical studies including in-home studies, studies with children exhibiting ADHD symptoms, and randomized controlled trials. Average post-treatment scores on the TAI in the current study (47.75) falls within the range of averages obtained in other studies (ranging 
from 44.1 to 49.6). Additionally, average mid-treatment ratings were similar to mid-treatment ratings obtained by Eisenstadt and colleagues (1993) for families who received CDI first.

\section{Discussion}

The overall goal of the current study was to extend the literature on the utility of PCIT with an ADHD population. This was accomplished by testing theoretical assumptions underlying skills taught in PCIT (i.e., effects of treatment on on-task behavior) and employing a multi-method assessment by administering measures important in the diagnosis of ADHD that are not routinely included in PCIT clinical practice or research (e.g., IRS, CTRS-RS). Findings are indicative of improvements in on-task behavior, reductions in ADHD symptoms and impairment, decreases in co-occurring disruptive behavior, and adequate consumer satisfaction with the overall treatment package (i.e., following completion of both CDI and PDI). Results pertaining to hypotheses and exploratory questions, limitations, and future directions are discussed below.

\section{Specific Hypotheses}

The primary observable hypothesis of the current study was that child on-task behavior would visibly increase from baseline during the CDI phase of the intervention and changes would persist or increase further during the second treatment phase, PDI, and during follow-up. Results from two of the four families enrolled in the study fully supported this hypothesis (i.e., initial visible increase occurred coinciding with the introduction of CDI, persisted during PDI, and maintained at follow-up). Specifically, both Phillip and Matthew exhibited an increase in level coinciding with the change from baseline to CDI. These gains persisted throughout the remainder of treatment and at follow-up; however, variability also increased during PDI. The reason for increased variability is unclear. It is possible that practice effects occurred with 
repeated experience with the coloring task. During this phase of treatment, both Phillip and Matthew's mother were skilled at providing social reinforcement to appropriate on-task behavior and using differential attention to decrease the frequency and duration of mild disruptive behaviors. Moreover, they were establishing and strengthening discipline skills. These skills may have attenuated the effects of repeated practice (i.e., increasing boredom with the task).

The results from two other participants (Jacob and Charlie) did not fully support the hypothesis. Jacob's results partially supported the hypothesis. In particular, he exhibited variable on-task behavior during CDI and did not demonstrate a visible increase from baseline coinciding with the introduction of CDI (e.g., lowest data point following introduction of CDI, 6 of 12 data points lower than or equal to lowest percentage at baseline). However, Jacob's ontask behavior did visibly increase coinciding with the introduction of PDI (i.e., increase in level, change in slope) and was maintained at follow-up (higher percentage at post-treatment and follow-up compared to baseline).

While Jacob's change in on-task behavior partially supported the hypothesis, the timing of his increase differed from Phillip and Matthew's increase in on-task behavior. It is possible that the function of Jacob's off-task behavior differed from Phillip and Matthew's off-task behavior. Interestingly, a recent study examining the function of home problem behavior for preschoolers ages 3-5 with or at-risk for ADHD found that escape was the primary function followed by tangibles, and then attention (Sokol, Kern, Arbolino, \& DuPaul, 2009). These researchers also found that most problem behaviors had multiple functions. As CDI theoretically primarily targets disruptive behaviors maintained by parental attention and PDI targets behaviors maintained by both attention and escape (McNeil, Filcheck, Greco, Ware, \& Bernard, 2001), it is possible that participants in the current study exhibited similar behavior topography (i.e., on- and 
off-task behavior at baseline) that differed in underlying function. Also, Jacob's mother was the only mother in the study to self-disclose an ADHD diagnosis. This possibly could have influenced her behavior during the 2 phases as coaching in CDI is less directive in comparison to PDI (Eyberg, 1999). Moreover, there is some evidence that maternal ADHD symptoms interfere with the efficacy of parent training (Sonuga-Barke et al., 2002). Researchers have recognized the need to evaluate more active and directive treatments such as PCIT with this population (Chronis et al., 2004). Alternatively, as Jacob was the only participant with this pattern of change in on-task behavior, it is possible that other factors (history or maturation) influenced results.

Charlie's on-task behavior during the study did not support the hypothesis. At baseline, Charlie's percentage of on-task behavior resembled comparison children without a disruptive behavior disorder (e.g., Jacobs et al., 2000; McNeil et al., 1991) and clinic-referred children following treatment (e.g., McNeil et al., 1991). Therefore, it is possible that measurement issues (i.e., ceiling effects at baseline) interfered with observable change following the experimental manipulation (i.e., introduction of CDI and PDI). Given Charlie's high percentage of on-task behavior at baseline, the researcher considered excluding him from the study. A decision was made to include him because he clearly met all other inclusion criteria, had one data point below $50 \%$ on-task behavior at baseline, and exhibited decreasing on-task behavior from baseline sessions 3-6, raising the possibility that initial high percentage rates were the result of novelty effects. Clinically, Charlie appeared to enjoy coloring more than any other participant in the study which may have influenced on-task behavior at baseline as children typically do not exhibit the same level of impairment in a setting at all times (APA, 2000). Although he was 
included in the study and completed treatment, his behavior at baseline may have limited the ability to observe visible effects coinciding with the introduction of treatment.

Overall, study findings based on observable changes and comparison with other research are promising. Findings suggest that PCIT may provide parents with skills to facilitate child ontask behavior. Changes in Phillip and Matthew's on-task behavior occurred at the introduction of CDI, which is consistent with the theorized effects of CDI skills and supported by the results of several analogue studies (Lemaster et al., 2010; Tempel, 2009). However, more research is needed to better understand the mechanism of change (i.e., facilitative skills versus structured discipline) and whether change is dependent on directly targeting the underlying function of offtask behavior. Also, study replications that assess task preference and experimentally manipulate tasks based on idiographic preferences may provide empirical evidence in support of Charlie's performance.

The other primary observable hypothesis regarding child out-of-area behavior was not supported by data for any participant in the current study. A review of study methods suggests that this hypothesis was more exploratory in comparison to on-task behavior and several methodological factors may have limited the utility of this measurement. A major limiting factor was that stability criteria guiding when to change treatment conditions were developed based on on-task behavior and maternal mastery of CDI/PDI skills. Subsequently, out-of-area behavior was decreasing for 2 of 4 participants (i.e., Phillip and Matthew) during baseline, which interfered with visual inspection. Specifically, the change occurring during baseline was consistent with the change hypothesized to occur with the introduction of CDI. Another limiting factor was that this behavior occurred at a low frequency across treatment phases and participants (i.e., floor effects). While this behavior occurred infrequently at baseline and during 
treatment conditions for 3 of 4 participants (i.e., Phillip, Matthew, and Jacob), Charlie's behavior was highly variable throughout the entire study.

It is possible that the age of children enrolled in the study may have influenced the outof-area observation. Specifically, hyperactivity and motor activity tend to decline with age (Lahey et al., 2004). The 2 children (Phillip and Matthew) with the lowest rates of out-of-area behavior both at baseline and across treatment conditions were 6 years old. These slightly older children may have experienced additional practice with classroom expectations (e.g., remaining seated) compared to Charlie and Jacob, who were younger and exhibited more variability.

Regardless of participant age, it is uncertain whether this out-of-area observation is useful in assessment and intervention research. As all participants clearly met criteria for ADHD at pretreatment, it is unclear whether this observation has specificity in identifying children exhibiting hyperactivity and impulsivity. Future PCIT or BPT studies interested in obtaining observational data of hyperactivity and impulsivity would benefit from further piloting as well as considering other approaches to quantify motor activity (e.g., actigraphy) as well as examining different task durations. In particular, as floor effects were evident for Phillip and Matthew, increasing the length of the task may elicit more out-of-area behavior and better approximate classroom expectations as 5- and 6-year olds are required to sit for longer amounts of time. In contrast to on-task behavior, which was piloted in 2 other studies examining the modified definition and coloring task procedure (Lemaster et al., 2010; Tempel, 2009), out-of-area behavior was not systematically piloted.

\section{Exploratory Questions}

Findings from the current study support the first exploratory question regarding parental ratings of child behavior. In particular, it was expected that parents would report reductions in 
ADHD symptoms at mid-treatment, post-treatment, and follow-up compared to pretreatment. In particular, all participants exhibited clinically-significant symptoms of inattention and/or hyperactivity/impulsivity on the CPRS-RS and the DSM-IV Structured Interview for Disruptive Behavior Disorders at pretreatment. Symptoms slightly decreased by mid-treatment on some subscales for all participants. By post-treatment, all participants were within normal limits on the CPRS-RS subscales including Cognitive Problems/Inattention and the ADHD Index. Aside from Phillip, all participants were below the clinical cutoff for Hyperactivity on this measure immediately following treatment. All participants maintained gains on the CPRS-RS at followup. A similar pattern was observed on the DSM-IV Structured Interview for Disruptive Behavior Disorders except that both Phillip and Matthew still exhibited a clinically-significant number of Hyperactivity/Impulsivity symptoms at post-treatment. However, they fell below this cutoff by follow-up. Charlie's gains in Inattention were not maintained at the follow-up session.

Overall, these findings are consistent with PCIT treatment outcome studies (e.g., Matos et al., 2006; Matos et al., 2009; Nixon, 2001) including 2 randomized controlled trials (i.e., Matos et al., 2009; Nixon, 2001) demonstrating clinically-significant decreases in ADHD symptoms. Moreover, these findings also are consistent with research on the effects of other evidence-based BPT programs (i.e., Triple P Positive Parenting Program and the Incredible Years) on symptoms of ADHD (Bor et al., 2002; Jones et al., 2007). Findings that clinically-significant changes in symptoms primarily occurred between mid-treatment and post-treatment are in contrast to changes in on-task behavior which occurred in CDI for 2 participants. Given that parents are only instructed to use skills during therapy sessions and during a 5-minute homework practice, changes in more global ratings of symptoms may occur in PDI when there is increasing emphasis on generalizing skills from formal therapy sessions and homework practices (Eyberg, 1999; 
McNeil \& Hembree-Kigin, 2010). All mothers completed homework more frequently during PDI compared to CDI which may have enhanced generalization. Additionally, problem behavior may have multiple functions and may be driven primarily by escape (Sokol et al., 2009), which may explain why larger gains were obtained between mid-treatment and post-treatment. Specifically, PDI targets escape-maintained behavior as children must comply with the original command following timeout for noncompliance. The only study directly examining the effects of the 2 stages of PCIT on hyperactivity did not find a difference between changes in hyperactivity across stages; however, found a significant reduction in symptoms following both stages, regardless of order (Eisenstadt et al., 1993). Thus, it is possible that positive parenting and discipline skills work in a complementary fashion to reduce symptoms of inattention and hyperactivity/impulsivity.

Despite these promising findings, it is possible that the reduction in symptoms of hyperactivity between pre- and post-treatment and post-treatment and follow-up was due to maturation and was not a direct effect of treatment. Specifically, hyperactive/impulsive symptoms are less stable in young children and almost one-third of children meeting criteria for this specific subtype do not meet diagnostic criteria 1 year later (Lahey et al., 2004). However, all participants completed all treatment sessions except the follow-up in 6 months or less. Also, the results of 2 randomized controlled trials comparing PCIT to a wait-list control group offer support that reductions in hyperactivity may be attributable to treatment and not maturation (Matos et al., 2009; Nixon, 2001). Therefore, Phillip and Matthew's mothers may have continued to practice skills between post-treatment and follow-up, resulting in further reductions in symptoms. Further, Boggs and colleagues (2004) compared families who completed PCIT with families who dropped out of treatment and found significantly fewer children who received 
treatment met symptom criteria for ADHD, which provides further support that observed effects are due to treatment and not merely maturation. Overall, these findings are consistent with previous research and are promising in the use of PCIT for ADHD symptoms.

Study findings also offer overall support of the researcher's initial expectation that parents will report reductions in child impairment in different domains at post-treatment and follow-up. Both Phillip and Jacob did not exhibit clinically-significant impairment on any domain at post-treatment and follow-up. Additionally, Matthew's scores on impairment in relationship with peers and relationship with parent fell below clinical significance at posttreatment and follow-up. Although maternal ratings of impairment at post-treatment showed that Charlie's scores fell below clinical significance in several domains (i.e., relationship with siblings, relationship with parents, and family functioning); none of these gains persisted.

Given treatment components, improvements in parent-child relationships and social skills with siblings and peers are consistent with theories underlying PCIT (Wagner \& McNeil, 2008). All maternal reports were indicative of reduced impairment in their relationship with parents, although this reduction did not persist for Charlie. Both participants with a sibling (Jacob and Charlie) did not exhibit maternal reported clinically-significant impairment in this domain at post-treatment, although Charlie's improvement again did not persist. Although Phillip and Matthew did not have siblings, Matthew's impairment with peers fell below the clinical cutoff at post-treatment and follow-up and Phillip did not exhibit impairment in this domain.

In terms of family functioning, improvements observed at post-treatment did not maintain at follow-up for 2 participants (Matthew and Charlie) even though both participants continued to experience reduced impairment in parent-child relationships. Both Matthew and Charlie also continued to exhibit impairment in academic progress, which may contribute to family 
functioning. Specifically, Charlie's mother was consulting lawyers and special education advocates regarding due process following numerous suspensions for aggressive behavior in school. Similarly, Matthew's mother had been notified of academic difficulty at the end of the academic year prior to post-treatment and was prepared to request an evaluation for special education services the following school year. It is possible that academic concerns influenced overall family functioning. Additionally, it is plausible that other variables such as maternal psychopathology, parenting stress, and marital satisfaction influenced this rating. Both parents who reported clinically-elevated impairment in family functioning disclosed psychopathology and relationship difficulties (i.e., Matthew's mother self-disclosed mood disturbances and problems in close familial relationships and Charlie's mother disclosed difficulties with anxiety and marital problems). Although Phillip and Jacob's mothers also reported psychological distress, both verbally expressed satisfaction in close relationships (Phillip's mother expressed satisfaction in current romantic relationship and Jacob's mother reported marital satisfaction). Therefore, it is plausible that the combination of multiple factors influence family functioning including child behavior, parental psychological adjustment, and parental close and intimate relationships.

Significant impairment in parent report of self-esteem was also noted by Matthew's and Charlie's mother at follow-up. Although improving self-esteem is a goal of CDI, this construct is difficult to measure in a quantifiable way, and therefore, changes in self-esteem are not required for mastery. However, consistent with theoretical underpinnings of PCIT, training parents to increase positive verbal behavior and decrease negative verbal behavior theoretically should influence feedback children receive about behavior and subsequently verbal labels they attribute to their abilities and performance. As both Matthew and Charlie were experiencing 
academic and/or behavioral impairment in school, it is possible that negative academic feedback influenced how they labeled their abilities and performance.

Based on maternal ratings of symptoms and impairment at post-treatment and follow-up, only Charlie exhibited clinically-significant symptoms and impairment at follow-up. While Matthew did not experience significant symptoms, he exhibited clinically-significant impairment (in 2 or more domains) at both post-treatment and follow-up. These findings highlight the importance of measuring both symptoms and impairment as children who exhibit sub-clinical levels of ADHD symptoms can still experience significant impairment necessitating clinical services (APA, 2000; Fabiano et al., 2006).

Unfortunately the current study was unable to systematically evaluate the hypothesis that teachers would report a reduction in child oppositional behavior but not ADHD behavior at posttreatment and follow-up. Although this hypothesis was not tested with standardized rating scales, there was evidence that gains did not generalize for 3 of 4 participants. Specifically, Charlie's oppositional difficulties at school escalated during treatment, resulting in suspensions from school and prompting his parents to seek due process. These difficulties escalated despite clinician's efforts to consult with teachers and the multidisciplinary team before his parents sought out assistance from advocates and legal counsel. Although less impairing compared to his oppositional symptoms, Charlie's teacher continued to verbally express concerns regarding ADHD symptoms (e.g., out of seat, off-task, and work completion) during treatment. Further the school was debating whether to promote him from kindergarten to first grade given both academic and behavioral concerns. Interestingly, Charlie's teacher rated his oppositional problems highest at pretreatment in comparison to teacher ratings for other participants. 
While other participants did not exhibit as severe oppositional problems as Charlie, there was still evidence of persisting impairment. For instance, Matthew completed treatment in the summer so teacher ratings were not obtained. However, his mother expressed receiving report of teacher concern with peer interactions, performance in writing and math, and work completion at the end of the school year. She was planning on pursuing an evaluation for special education services the subsequent school year. Also, the clinician consulted with Jacob's parents and teacher during PDI to develop a home-school note given persistent problems in school (i.e., out of seat, making noises, touching others). This clinical decision was made based on parental distress and because timing of enrollment prevented collecting teacher ratings (i.e., different teacher completed pretreatment ratings as he began the study at the end of a school year). While his parents reported gains with this intervention, it is worth noting that they were reconsidering their decision on medication at follow-up due solely to concerns at school. Based on this information, it appears that PCIT in isolation was insufficient in addressing the behavioral and academic concerns for at least 3 of the 4 participants. Although this finding is based on clinical evidence and not systematic standardized measurement, it is not unexpected given the literature. In particular, Deault (2009) reviewed literature on the relation between parenting and impairment in families with a child with ADHD and concluded that there is a paucity of research on the association between parents and academic achievement. Further research on school generalization suggests that gains related to ADHD symptoms may not generalize following PCIT (McNeil et al., 1991).

As expected, mothers reported decreases in disruptive behavior from pretreatment to post-treatment and follow-up on the CPRS-RS, DSM-IV Structured Interview for Disruptive Behavior Disorders, and ECBI. Levels of oppositional behavior were below the clinical cutoff 
across all measures for all participants at post-treatment. Gains persisted at follow-up aside from Charlie's mother's rating on the Problem Scale of the ECBI, which placed him above the clinical cutoff. Study findings are consistent with PCIT treatment outcome research (e.g., McNeil et al., 1991; Schuhmann et al., 1998) and results of a meta-analysis showing large effects on parent report of child behavior following intervention (Thomas \& Zimmer-Gembeck, 2007). Changes on the ECBI in the current study were larger than changes observed in wait-listed controls (Schuhmann et al.; Nixon, 2003). Overall, the current study found larger reductions on the ECBI in comparison to the published literature. However, the changes were similar to another homebased PCIT study (Ware et al., 2008). It is plausible that providing treatment in the home facilitates skill acquisition and use (Timmer, Zebell, \& Culver, 2010). Another alternative explanation for the large reductions is that participant desire to please the experimenter may be higher when the clinical researcher enters the family's home to intervene and collect data. The researcher attempted to minimize that effect by emphasizing that this study is one of the first to examine the effects of the treatment on ADHD and participant responses would help researchers further develop and evaluate modifications for families of children with ADHD. Additionally, the researcher interacted with the child and allowed mothers to complete assessments independently after providing initial instructions. Another factor that may contribute to the large improvement on the ECBI is treatment dose. Specifically, families received more treatment sessions in the current study than in typical PCIT treatment studies. While some of the outcome studies specified a set number of sessions (e.g., Brestan et al., 1997; Chaffin et al., 2004; Eisenstadt et al., 1993; McNeil et al., 1991; Nixon et al., 2000), investigations utilizing standard mastery criteria have reported an average number of sessions ranging from 13 to 18.7 (e.g., McCabe \& Yeh, 2009; Schuhmann et al., 1998; Timmer et al., 2005). 
Current findings pertaining to the exploratory question regarding parenting stress are inconclusive. All parents reported elevations on 1-3 (of 3 possible) subscales on the PSI-SF at pretreatment consistent with research documenting increased stress in parents of children with ADHD, particularly among parents of younger children (Fischer, 1990; Mash \& Johnson, 1983; Wells et al., 2000). However, all mothers who reported elevations on the PSI-SF on multiple subscales continued to report scores above the cutoff at both post-treatment and follow-up. These mixed-findings parallel research findings on the effects of BPT on maternal stress (e.g., Anastopoulos, Shelton, DuPaul \& Guevremont, 1993; Bor et al., 2002; Danforth et al., 2006; Pisterman et al., 1989). Similarly, Wells and colleagues (2000) did not find a significant reduction in parenting stress in any group in the MTA (Medication Management, Behavior Management, Combined Treatment, and Community Care). Bor and colleagues (2002) looked at the effects of Triple $\mathrm{P}$ on parenting stress in an ADHD preschool sample, also failing to find a significant difference. These findings contrast PCIT treatment outcome research showing significant reductions in parental stress (e.g., Eisenstadt et al., 1993; Eyberg et al., 2000; McCabe \& Yeh, 2009; Schuhmann et al., 1998). However, many of these studies included samples of children with primarily oppositional problems, potentially influencing results. Overall, researchers and clinicians have recognized that specific characteristics of children with ADHD influence parenting stress (Barkley, 1985). In particular, these children require greater supervision and control, do not respond well to typical methods of child behavior management, and have difficulty conforming to rule-governed behavior. Subsequently, ADHD alone is associated with impaired family functioning and parental stress with co-occurring ODD and CD heightening difficulties (e.g., Deault, 2010; Gadow \& Nolan, 2002). As the degree of comorbid ADHD and ODD was higher in the current study than the majority of PCIT treatment outcome 
research (Wagner \& McNeil, 2008), the effects of treatment on parental stress may be lower. It is also important to note that the 2 mothers who did not endorse significant stress on the PSI at post-treatment still exhibited stress and distress during the course of treatment. Specifically, clinicians worked with Phillip's mother on relaxation during PDI and Jacob's mother on increasing pleasant events to manage stress during CDI. While these discussions are not unusual during PCIT or a deviation of the protocol as there is a specific handout utilized in CDI entitled Kids and Stress (Eyberg, 1999), it is interesting to note the high levels of distress in the current study which is consistent with the research on parenting children with ADHD.

The final question explored in this study was whether parental report of treatment satisfaction would indicate that the treatment is acceptable for parents of children with ADHD based on assessment at mid-treatment, post-treatment, and follow-up. This hypothesis was supported as findings parallel PCIT research with children with ADHD (Matos et al., 2006; Matos et al., 2009), children with DBD (e.g., Schuhmann et al., 1998), and home-based service delivery (Masse, 2009; Ware et al., 2008). Further findings at mid-treatment are supported by evidence that parents rate CDI as less favorable compared to ratings of PDI or the entire treatment package (Eisenstadt et al., 1993).

\section{Additional Limitations}

While findings from the current project overall offer support for the utility of PCIT with children with ADHD, there are a number of limiting factors worth noting in addition to issues discussed in the context of explaining findings above. These limitations pertain to the design sample, treatment, and measurement. A major limiting factor is that this study employed a nonconcurrent design to examine the effects of treatment. This design was selected due to practical and feasibility issues surrounding recruiting a clinical sample of young children from a small 
city. Compared to other single subject designs, multiple baseline designs across participants have reduced ability to control for threats to internal validity. Further, non-concurrent baseline designs are less rigorous (i.e., increased risk of a historical event influencing the dependent variable instead of the treatment). While different events may have influenced individual participant's behavior at various times during treatment (e.g., Christmas, end of the school year, and family vacation), no known events coincided with a phase change. Also, treatment between participants overlapped as data were being collected for multiple participants simultaneously, which may have lessened the threat.

Another limiting factor that may restrict the generalization of study findings is the relatively homogenous sample with regard to demographic characteristics (e.g., child gender, race, age, and parental involvement). In particular, all children participating in the study were male. Research documents a 2:1 gender ratio in ADHD in young children which is comparable to rates observed in school-age children (Pelham et al., 2004). A recent study examining a community sample of 7964 -year olds found rates of ADHD of $14.6 \%$ for boys and $9 \%$ for girls respectively. Therefore, the current study was not representative of the population (i.e., 1-2 females would be expected to participate given the gender ratio). Some research suggests that girls with ADHD are under identified and less likely to receive services (Froehlich et al., 2007). Although there is not literature suggesting that treatment effects would differ across males and females (e.g., The MTA Cooperative Group, 1999), replication with female participants is warranted.

Additionally, all participants were White males even though prevalence rates suggest a higher rate of ADHD in African American and Hispanic 4 year olds (Lavigne, LeBailly, Hopkins, Gouze, \& Binns, 2009). Similar to gender, race/ethnicity influences service utilization. 
There is evidence that ADHD may be under diagnosed and treated in minority populations (e.g., Cox, Motheral, Henderson, \& Mager, 2003). Inclusion criteria in the current study included a prior diagnosis of ADHD, which may have influenced the ability to enroll females and minority children. It is unclear whether racial and ethnic diversity influences treatment results. Several researchers found positive effects on ADHD symptoms in Hispanic samples without making cultural adaptations (Matos et al., 2006, 2009; McCabe \& Yeh, 2009). Also, research suggests that African American and White families who receive treatment matched for SES may not differ in terms of outcome (Capage, Bennett, \& McNeil, 2001).

Another demographic limitation was the age of children as all children who completed the study were at the upper end of the age range and none were enrolled in preschool. Although preschool children were targeted in recruitment through the distribution of ads, none contacted the researcher, expressed interest, and completed the pretreatment assessment. Research has found low rates of mental health service utilization among 4-year olds with psychopathology (Lavigne et al., 2009) with rates increasing to approximately $20 \%$ for school age children (Lavigne et al., 1998; Pavuluri, Luk, \& McGee, 1996). Therefore Lavigne and colleagues (2009) have suggested that entry into schooling may prompt families to seek services and highlights the need to address barriers to early screening and intervention.

The specific exclusion of fathers in the study also poses a limitation. Bagner and Eyberg (2003) found that father participation in treatment may help maintain gains. Given that ADHD is a chronic disorder and requires constant intervention (i.e., continued medication and/or continued implementation of behavior modification), father involvement may be particularly advantageous. Research suggests different models of delivery (e.g., implementing behavioral parent training in the contexts of sports) may increase father engagement (Fabiano et al., 2009); therefore, further 
research would benefit from examining different contexts that may enhance the engagement of both parents in PCIT and other BPT programs.

Other major study limitations pertain to treatment. In particular, the dose of treatment was considerably higher in the current study (i.e., number of sessions) than in other PCIT studies. It is likely that the design in which additional sessions of CDI and PDI were conducted following mastery if stability criteria had not been met and in-home treatment delivery (e.g., Masse, 2009) influenced the duration of treatment. Additionally, maternal psychopathology may have influenced homework completion, skill acquisition, and progress (Sonuga-Barke et al., 2002). Further research examining maternal psychopathology in PCIT research is warranted given the association between ADHD in young children and parental ADHD, anxiety, depression, and substance use (Chronis, Lahey, Pelham, Kipp, Baumann, \& Lee, 2003). Despite this possibility, it is important to note that participants in the current study received a larger treatment dose than studies employing a set number of sessions (e.g., Eisenstadt et al., 1993; Nixon, 2001) and one randomized controlled trial in which families averaged 13 sessions (Schuhmann et al., 1998). It is unclear the extent to which treatment is effective at lower doses. Another limitation of the design was that participants were compensated for completed assessments which may have also influenced results on rating scales. Although the use of observations and researcher instructions may have minimized this limitation, it is unclear how this compensation influenced maternal report.

Additional study limiting factors involved the measurement of behavioral observations. All measurements occurred in environments with considerable distractions (i.e., home; Masse \& McNeil, 2008) observing children who, by diagnostic criteria, are particularly prone to distractions (APA, 2000). Efforts were made to minimize distractions by only allowing parent- 
child dyad to attend sessions, using standard toys brought by researchers into the home, collecting data in the same room every session, requesting that parents silence their phone, and addressing other distractions on an individual basis; however, individual environmental distractions were noted across participants. Some examples include: Phillip locked himself in his room one session, Charlie ran outside once during timeout, Charlie ran to the window every time a four-wheeler drove by outside, Matthew and Jacob's mother's phone rang several times, a neighbor came to the door at Charlie's home, and Matthew's dog ran around the room during several sessions. Subsequently, collecting data in the home may have increased the external validity of the current study at the expense of decreased experimental control. Another factor influencing measurement in the home was historical events. For instance, Charlie was disruptive and off-task during CDI 3 after his parents had just decorated their home for Christmas. During this session, he was not interested in playing or coloring with his mother and instead attempted to show researchers decorations during coding. A final important measurement limitation is the majority of behavioral observations were conducted by clinical researchers who had extensive contact with families and were aware of study hypotheses. While $20 \%$ of formal assessment observations were coded for reliability by an observer unaware of the assessment session (i.e., pre, mid, post, or follow-up), it is unclear if observations during the course of therapy (not part of formal assessment) were influenced by these factors.

\section{Future Directions}

Although findings are promising, this project highlighted a number of areas in need of future empirical investigation. Specifically, the academic and behavioral difficulties present in young children with ADHD are concerning. Future projects should examine the additive effects of intervening simultaneously in the school to provide classroom contingency management and 
academic evaluation and intervention. While these components are typically employed with older school-age children (Pelham \& Fabiano, 2008; Pelham, et al., 1998), there is less research targeting these domains with younger children. In particular, children in the PATS study were enrolled in the medication trial if they did not respond to parent training and intensive services were not provided in the school (Greenhill et al., 2006). Given the chronic nature of ADHD, it may be beneficial to intervene in daycares and preschools when treating at-risk children or children who have recently been diagnosed. Using interventions such as Teacher-Child Interaction Therapy (TCIT) or other classroom behavioral interventions may help provide prevention or early intervention services (e.g., TCIT as universal prevention; Gershenson, Lyon, \& Budd, 2010). Similarly, the current study enrolled children who exhibited severe symptoms and impairment as 3 of the children were on stimulant medication, 2 clearly met diagnostic criteria for ODD based on the DSM-IV Structured Interview for Disruptive Behavior Disorders, and the other 2 were slightly below the threshold but had clinically-significant elevations on subscales measuring disruptive behavior. While these characteristics predict diagnostic stability over time (e.g., Todd et al., 2008), they may also influence the course of treatment. Researchers employing longitudinal designs and utilizing comprehensive assessment strategies would provide valuable information in regard to the course of development for children who receive treatment when they are initially sub-clinical or at-risk for ADHD. Also, longitudinal designs could evaluate different methods of follow-up (i.e., booster sessions). As ADHD is a chronic disorder, researchers have recognized the need for long-term care similar to chronic care or dental care models (e.g., Van Cleave \& Leslie, 2008). This need is particularly salient given findings from the 8-year follow-up of children enrolled in the MTA (Molina et al., 2009). While Van Cleave and Leslie (2008) outline a model of chronic care that could be applied to childhood ADHD with 
routine visits scheduled every 3-6 months, there is a paucity of empirical evidence on how to provide efficacious, efficient, and cost effective long-term care.

Another area in need of further treatment is parental psychopathology and stress. Even though all children showed improvement over the course of the study in terms of symptoms and impairment, stress remained clinically-elevated for 2 mothers. Also, all mothers self-disclosed psychopathology. Maternal depression was predictive of worse outcomes at 36 months for children participating in the MTA study (Owens et al., 2003). While some researchers have specifically targeted maternal depression and BPT (e.g., Chronis, Gamble, Roberts \& Pelham, 2006) and the stress experienced by single parents and ADHD (e.g., Chacko et al., 2009), there is a lack of evidence on how to assess for a wide-range of parental psychopathology and target efficiently and effectively in the context of treating children with externalizing disorders. Specific interventions targeting parenting stress may be beneficial (Treacy, Tripp, \& Baird, 2005). Alternatively, addressing child impairment and skill deficits may have the potential to reduce stress. It is possible that targeting school and home problems simultaneously will reduce stress. Stress may also be associated with the difficulty of providing high intensity behavior modification over the long-term. Subsequently, research examining teaching children specific skills such as organizational or independent play skills may provide parents respite, thus alleviating parenting stress. For instance, Binder, Dixon, and Ghezzi (2000) successfully increased self-control defined as the ability to wait for a large reinforcer instead of receiving an immediate small reinforcer, in three children ages 3-5 with ADHD by teaching them verbal activities to engage in during the delay. It is unclear whether similar procedures could be employed to teach independent on-task behavior during play.

\section{Conclusions}


This study provides empirical support for the utility of PCIT in reducing symptoms and impairment associated with ADHD as well as co-occurring disruptive behaviors. Replication is needed to address study limitations pertaining to the sample and dose of treatment. Additional PCIT research would benefit from examining how to effectively incorporate school consultation, management of parental stress and psychopathology, and long-term monitoring and care when indicated in an efficient manner for this population. 


\section{References}

Abidin, R. R. (1995). Parenting stress index 3rd edition: Professional manual. Lutz, FL: Psychological Assessment Resources, Inc.

Achenbach, T. M. (1991). Manual for the Child Behavior Checklist/4-18 and 1991 Profile. Burlington: University of Vermont, Department of Psychiatry.

American Psychiatric Association. (2000). Diagnostic and statistical manual of mental disorders DSM-IV-TR (Text Revision). Washington, DC: Author.

Anastopoulos, A. D., Shelton, T. L., DuPaul, G. J., \& Guevremont, D. C. (1993). Parent training for attention deficit hyperactivity disorder: Its impact on parent functioning. Journal of Abnormal Child Psychology, 21(5), 581-596. doi: 10.1007/BF00916320

August, G. J., Realmuto, G. M., MacDonald, A. W., \& Nugent, S. M. (1996). Prevalence of ADHD and comorbid disorders among elementary school children screened for disruptive behavior. Journal of Abnormal Child Psychology, 24(5), 571-595. doi: 10.1007/BF01670101

Bagner, D. M., \& Eyberg, S. M. (2003). Father involvement in parent training: When does it matter? Journal of Clinical Child and Adolescent Psychology, 32(4), 599-605. doi: 10.1207/S15374424JCCP3204

Bagner, D. M., \& Eyberg, S. M. (2007). Parent-child interaction therapy for disruptive behavior in children with mental retardation: A randomized controlled trial. Journal of Clinical Child and Adolescent Psychology, 36(3), 418-429. Retrieved from: http://www.informaworld.com/smpp/title $\sim$ content=t775648094 
Bahl, A. B., Spaulding, S. A., \& McNeil, C. B. (1999). Treatment of noncompliance using parent-child interaction therapy: A data-driven approach. Education and Treatment of Children, 22(2), 146-156.

Barkley, R. A. (1985). The social behavior of hyperactive children: Developmental changes, drug effects, and situational variation. In R. McMahon \& R. Peters (Eds.), Childhood disorders: Behavioral-developmental approaches (pp. 218-243). New York: Brunner/Mazel.

Barkley, R. A. (1997). Attention-deficit/hyperactivity disorder. In E. J. Mash \& R. A. Barkley (Eds.), Treatment of childhood disorders (2nd ed., pp. 55-110). New York: Guilford Press.

Barkley, R. A. (1998). Attention-deficit/hyperactivity disorder: A handbookfor diagnosis and treatment (2nd ed.). New York: Guilford Press.

Barkley, R. A. (2002). Psychosocial treatments for attention-deficit/hyperactivity disorder in children. Journal of Clinical Psychiatry, 63(Suppl 12), 36-43. Retrieved from: http://www.psychiatrist.com/default2.asp

Barkley, R. A., Fischer, M., Smallish, L., \& Fletcher, K. (2006). Young adult outcome of hyperactive children: Adaptive functioning in major life activities. Journal of American Academy of Child and Adolescent Psychiatry, 45(2), 192-196. doi:

10.1097/01.chi.0000189134.97436.e2

Barkley, R. A., Murphy, K., \& Bauermeister, J. J. (1998). Trastorno por Deficit de Atencion: Un Manual de Trabajo Clinico. New York: The Guilford Press.

Barlow, D. H., \& Hersen, M. (1984). Multiple baseline designs. In D.H. Barlow \& M.D. Hersen, Single case experimental designs (2nd ed.) (pp. 209-251). New York: Pergamon. 
Binder, L. M., Dixon, M. R., \& Ghezzi, P. M. (2000). A procedure to teach self-control to children with attention deficit hyperactivity disorder. Journal of Applied Behavior Analysis, 33(2), 233-237. doi: 10.1901/jaba.2000.33-233

Boggs, S. R., Eyberg, S. M., Edwards, D. L., Rayfield, A., Jacobs, J., Bagner, D., \& Hood, K. K. (2004). Outcomes of parent-child interaction therapy: A comparison of treatment completers and study dropouts one to three years later. Child and Family Behavior Therapy, 26(4), 1-22. doi: 10.1300/J019v26n04_01

Bor, W., Sanders, M. R., \& Markie-Dadds, C. (2002). The effects of the triple p-positive parenting program on preschool children with co-occurring disruptive behavior and attentional/hyperactive difficulties. Journal of Abnormal Child Psychology, 30(6), 571587. doi: 10.1023/A:1020807613155

Brestan, E. V., Eyberg, S. M., Boggs, S. R., \& Algina, J. (1997). Parent-child interaction therapy: Parents' perceptions of untreated siblings. Child and Family Behavior Therapy, 19(3), 13-28. doi: 10.1300/J019v19n03_02

Brestan, E. V., Jacobs, J. R., Rayfield, A. D., \& Eyberg, S. M. (1999). A consumer satisfaction measure for parent-child treatments and its relation to measures of child behavior change. Behavior Therapy, 30(1), 17-30. doi: 10.1016/S0005-7894(99)80043-4

Campbell, S. B. (1995). Behavior problems in preschool children: A review of recent research. Journal of Child Psychology and Psychiatry, 36(1), 113-149. doi: 10.1111/j.14697610.1995.tb01657.x

Campbell, S. B. (2002). Behavior problems in preschool children: Clinical and developmental issues (2nd ed.). New York: Guilford Press. 
Campbell, S. B., Ewing, L. J., Breaux, A. M., \& Szumowski, E. K. (1986). Parent-referred problem three-year olds: Follow-up at school entry. Journal of Child Psychology and Psychiatry, 27(4), 473-488. doi: 10.1111/j.1469-7610.1986.tb00635.x

Cantwell, D. P., \& Baker, L. (1989). Stability and natural history of DSM-III childhood diagnoses. Journal of the American Academy of Child and Adolescent Psychiatry, 28(5), 691-700. doi: 10.1097/00004583-198909000-00009

Capage, L. C., Bennett, G. M., \& McNeil, C. B. (2001). A comparison between African American and Caucasian children referred for treatment of disruptive behavior disorders. Child and Family Behavior Therapy, 23(1), 1-14. doi: 10.1300/J019v23n01_01

Chacko, A., Wymbs, B. T., Wymbs, F. A., Pelham, W. E., Swanger-Gagne, M. S., Girio, E., ... O’Connor, B. (2009). Enhancing traditional behavioral parent training for single mothers of children with ADHD. Journal of Clinical Child and Adolescent Psychology, 38(2), 206-218. doi: 10.1080/15374410802698388.

Chaffin, M., Silovsky, J. F., Funderburk, B., Valle, L. A., Brestan, E. V., Balachova, T., ... Bonner, B. L. (2004). Parent-child interaction therapy with physically abusive parents: Efficacy for reducing future abuse reports. Journal of Consulting and Clinical Psychology, 72(3), 500-510. doi: 10.1037/0022-006X.72.3.500

Choate, M. L., Pincus, D. B., Eyberg, S. M., \& Barlow, D. (2005). Parent-child interaction therapy for treatment of separation anxiety disorder in young children: A pilot study. Cognitive and Behavioral Practice, 12(1), 126-135. doi: 10.1016/S1077-7229(05)800471

Chronis, A. M., Chacko, A., Fabiano, G. A., Wymbs, B. T., \& Pelham, W. E. (2004). Enhancements to the behavioral parent training paradigm for families of children with 
ADHD: Review and future directions. Clinical Child and Family Psychology Review, 7(1), 1-27. doi: 10.1023/B:CCFP.0000020190.60808.a4

Chronis, A. M., Gamble, S. A., Roberts, J. E., \& Pelham, W. E. (2006). Cognitive-behavioral depression treatment for mothers of children with attention-deficit/hyperactivity disorder. Behavior Therapy, 37(2), 143-158. doi: 10.1016/j.beth.2005.08.001

Chronis, A. M., Lahey, B. B., Pelham, W. E., Kipp, H. L., Baumann, B. L., \& Lee, S. S. (2003). Psychopathology and substance abuse in parents of young children with attentiondeficit/hyperactivity disorder. Journal of the American Academy of Child and Adolescent Psychiatry, 42(12), 1424-1432. doi: 10.1097/00004583-200312000-00009

Chronis, A. M., Jones, H. A., \& Raggi, V. L. (2006). Evidence-based psychosocial treatments for children and adolescents with attention-deficit/hyperactivity disorder. Clinical Psychology Review, 26(4), 486-502. doi: 10.1016/j.cpr.2006.01.002

Coie, J. D., \& Dodge, K. A. (1998). Aggression and antisocial behavior. In W. Damon (Series Ed.) \& N. Eisenberg (Vol. Ed.), Handbook of child psychology. Vol. 3. Social, emotional, and personality development (5th ed., pp. 779-862). Hoboken, New Jersey: John Wiley \& Sons.

Conners, C. K. (1997). Technical manual for the Conners' Rating Scales - Revised. MultiHealth Systems Inc.

Cooley, E. L., \& Morris, R. D. (1990). Attention in children: A neuropsychologically based model for assessment. Developmental Neuropsychology, 6(3), 239-274. doi: $10.1080 / 87565649009540465$

Cox, E. R., Motheral, B. R., Henderson, R. R., \& Mager, D. (2003). Geographic variation in the prevalence of stimulant medication use among children 5 to 14 years old: Results from a 
commercially insured US sample. Pediatrics, 111(2), 237-243. doi: 10.1542/peds.111.2.237

Cunningham, C. E., Bremner, R., \& Boyle, M. (1995). Large group community-based parenting programs for families of preschoolers at risk for behavior disorders: Utilization, cost effectiveness, and outcome. Journal of Child Psychology and Psychiatry, 36(7), 11411159. doi: 10.1111/j.1469-7610.1995.tb01362.x

Danforth, J. S., Harvey, E., Ulaszek, W. R., \& McKee, T. E. (2006). The outcome of group parent training for families of children with attention-deficit hyperactivity disorder and defiant/aggressive behavior. Journal of Behavior Therapy and Experimental Psychiatry, 37(3), 188-205. doi: 10.1016/j.jbtep.2005.05.009

Deault, L. C. (2009). A systematic review of parenting in relation to the development of comorbidites and functional impairments in children with attention-deficit/hyperactivity disorder (ADHD). Child Psychiatry and Human Development, 41(2), 168-192. doi: 10.1007/s10578-009-0159-4

Dishion, T. J., McCord, J., \& Poulin, F. (1999). When interventions harm: Peer groups and problem behavior. American Psychologist, 54(9), 755-764. doi: 10.1037/0003066X.54.9.755

Eisenstadt, T. H., Eyberg, S., McNeil, C. B., Newcomb, K., \& Funderburk, B. (1993). Parentchild interaction therapy with behavior problem children: Relative effectiveness of two stages and overall treatment outcome. Journal of Clinical Child Psychology, 22(1), 4251. doi: $10.1207 / \mathrm{s} 15374424 \mathrm{jccp} 2201 \_4$ 
Erhardt, D., \& Baker, B. L. (1990). The effects of behavioral parent training on families with young hyperactive children. Journal of Behavior Therapy and Experimental Psychiatry, 21(2), 121-132. doi: 10.1016/0005-7916(90)90017-F

Evans, S. W., Schultz, B. K., \& Sadler, J. M. (2008). Safety and efficacy of psychosocial interventions used to treat children with attention-deficit/hyperactivity disorder. Psychiatric Annals, 38(1), 58-64. doi: 10.3928/00485713-20080101-02

Eyberg, S. M. (1974). Therapy Attitude Inventory. (Available from Sheila Eyberg, PhD, Department of Clinical and Health Psychology, Box 100165 Health Science Center, University of Florida, Gainesville, FL 32610-0165).

Eyberg, S. M. (1998). Parent-child interaction therapy: Integration of traditional and behavioral concerns. Child and Family Behavior Therapy, 10, 33-46.

Eyberg, S. M. (1999). Parent-Child Interaction Therapy Integrity Checklists and Session Materials. University of Florida.

Eyberg, S. M., Funderburk, B. W., Hembree-Kirgin, T. L., McNeil, C. B., Querido, J. G., \& Hood, K. K. (2001). Parent-child interaction therapy with behavior problem children: One and two year maintenance of treatment effects in the family. Child and Family Behavior Therapy, 23(4), 1-20. doi: 10.1300/J019v23n04

Eyberg, S. M., Nelson, M. M., Duke, M., \& Boggs, S. R. (2005). Manual for the Dyadic Parent-Child Interaction Coding System Third Edition. University of Florida.

Eyberg, S. M., Nelson, M. M., \& Boggs, S. R. (2008). Evidence-based treatments for children and adolescents with disruptive behavior. Journal of Clinical Child and Adolescent Psychology, 37(1), 215-237. doi: 10.1080/15374410701820117

Eyberg, S. M., \& Pincus, D. (1999). Eyberg Child Behavior Inventory Sutter Student Behavior 
Inventory - Revised professional manual. Psychological Assessment Resources.

Fabiano, G. A., Chacko, A., Pelham, W. E., Robb, J., Walker, K. S., Wymbs, F., ... Pirvics, L. (2009). A comparison of behavioral parent training programs for fathers of children with attention-deficit/hyperactivity disorder. Behavior Therapy, 40(2), 190-204. doi: 10.1016/j.beth.2008.05.002

Fabiano, G. A., Pelham, W. E., Waschbusch, D. A., Gnagy, E. M., Lahey, B. B., Chronis, A. M., ... Burrows-MacLean, L. (2006). A practical measure of impairment: Psychometric properties of the impairment rating scale in samples of children with attention deficit hyperactivity disorder and two school-based samples. Journal of Clinical Child and Adolescent Psychology, 35(3). 369-385. doi: 10.1207/s15374424jccp3503_3

Fischer, M. (1990). Parenting stress and the child with attention deficit hyperactivity disorder. Journal of Clinical Child Psychology, 19(4), 337-346. doi: 10.1207/s15374424jccp1904_5

Frankel, F., Myatt, R., Cantwell, D. P., \& Feinberg, D. T. (1997). Parent-assisted transfer of children's social skills training: Effects on children with and without attention-deficit hyperactivity disorder. Journal of the American Academy of Child and Adolescent Psychiatry, 36(8), 1056-1064. doi: 10.1097/00004583-199708000-00013

Froehlich, T. E., Lanphear, B. P., Epstein, J. N., Barbaresi, W. J., Katusic, S. K., \& Kahn, R. S. (2007). Prevalence, recognition, and treatment of attention-deficit/hyperactivity disorder in a national sample of US children. Archives of Pediatric and Adolescent Medicine, 161(9), 857-864. doi: 10.1001/archpedi.161.9.857

Funderburk, B. W., Eyberg, S. M., Newcomb, K., McNeil, C. B., Hembree-Kigin, T., \& Capage, L. (1998). Parent-child interaction therapy with behavior problem children: 
Maintenance of treatment effects in the school setting. Child and Family Behavior Therapy, 20(2), 17-38. doi: 10.1300/J019v20n02

Gadow, K. D., \& Nolan, E. E. (2002). Differences between preschool children with ODD, ADHD, and ODD + ADHD symptoms. Journal of Child Psychology and Psychiatry, 43(2), 191-201. doi: 10.1111/1469-7610.00012

Gadow, K. D., \& Sprafkin, J. (1997). Early Childhood Inventory - 4 norms manual. Los Angelos, CA: Western Psychological Services.

Gershenson, R. A., Lyon, A. R., \& Budd, K. S. (2010). Promoting positive interactions in the classroom: Adapting parent-child interaction therapy as a universal prevention program. Education and Treatment of Children, 33(2), 261-287. doi: 10.1353/etc.0.0092

Gilchrist, R. H., \& Arnold, E. (2008). Long-term efficacy of ADHD pharmacotherapy in children. Psychiatric Annals, 38(1), 52-57. doi: 10.3928/00485713-20080101-03

Goldfine, M. E., Wagner, S. M., Branstetter, S. A., McNeil, C. B. (2008). Parent-child interaction therapy: An examination of cost effectiveness. Journal of Early and Intensive Behavior Intervention, 5(1), 119-141. Retrieved from http://www.jeibi.com/

Goyette, C. H., Conners, C. K., \& Ulrich, R. F. (1978). Normative data on Revised Conners Parent and Teacher Rating Scales. Journal of Abnormal Child Psychology, 6(2), 221236. doi: 10.1007/BF00919127

Greenhill, L., Kollins, S., Abikoff, H., McCracken, J., Riddle, M, Swanson, J., ... Cooper, T. (2006). Efficacy and safety of immediate-release methylphenidate treatment for preschoolers with ADHD. Journal of the American Academy of Child and Adolescent Psychiatry, 45(11), 1284-1293. doi: 10.1097/01.chi.0000235077.32661.61 
Hanf, C. (1969). A two-stage program for modifying maternal controlling during mother-child $(M-C)$ interaction. Paper presented at the meeting of the Western Psychological Association, Vancouver, British Columbia, Canada.

Hartman, R. R., Stage, S. A., \& Webster-Stratton, C. (2003). A growth curve analysis of parent training outcomes: Examining the influence of child risk factors (inattention, impulsivity, and hyperactivity problems), parental and family risk factors. Journal of Child Psychology and Psychiatry, 44(3), 388-398. doi: 10.1111/1469-7610.00129

Harwood, M. D., \& Eyberg, S. M. (2004). Therapist verbal behavior early in treatment: Relation to successful completion of parent-child interaction therapy. Journal of Clinical Child and Adolescent Psychology, 33(3), 601-612. doi: 10.1207/s15374424jccp3303

Hinshaw, S. P., \& Melnick, S. M. (1995). Peer relationships in boys with attention-deficit hyperactivity disorder with and without comorbid aggression. Development and Psychopathology, 7(4), 627-647. doi: 10.1017/S0954579400006751

Hood, K. K., \& Eyberg, S. M. (2003). Outcomes of parent-child interaction therapy: Mothers' reports of maintenance three to six years after treatment. Journal of Clinical Child and Adolescent Psychology, 32(3), 419-429. doi: 10.1207/S15374424JCCP3203

Hoza, B., Kaiser, N. M., \& Hurt, E. (2007). Multimodal treatments for childhood attentiondeficit/hyperactivity disorder: Interpreting outcomes in the context of study designs. Clinical Child and Family Psychology Review, 10(4), 318-334. doi: 10.1007/s10567007-0025-5

Jacobs, J. R., Boggs, S. R., Eyberg, S. M., Edwards, D., Durning, P., Querido, J. G., ..., Funderburk, B. W. (2000). Psychometric properties and reference point data for the 
revised edition of the school observation coding system. Behavior Therapy, 31(4), 695712. doi: 10.1016/S0005-7894(00)80039-8

Jensen, P. S., Arnold, L. E., Swanson, J. M., Vitiello, B., Abikoff, H. B., Grenhill, L. L., ... Hur, K. (2007). 3-year follow-up of the NIMH MTA study. Journal of the American Academy of Child and Adolescent Psychiatry, 46(8), 989-1002. doi:

10.1097/CHI.0b013e3180686d48

Jensen, P. S., Hinshaw, S. P., Kraemer, H. C., Lenora, N., Newcorn, J. H., Abikoff, H. B., ... Vitiello, B. (2001). ADHD comorbidity findings from the MTA study: Comparing comorbid subgroups. Journal of the American Academy of Child and Adolescent Psychiatry, 40(2), 147-158. doi: 10.1097/00004583-200102000-00009

Johnson, B. D., Franklin, L. C., Hall, K., \& Prieto, L. R. (2000). Parent training through play: Parent-child interaction therapy with a hyperactive child. The Family Journal: Counseling and Therapy for Couples and Families, 8(2), 180-186. doi: $10.1177 / 1066480700082013$

Jurbergs, N., Palcic, J., \& Kelley, M. S. (2007). School-home notes with and without response cost: Increasing attention and academic performance in low-income children with attention-deficit/hyperactivity disorder. School Psychology Quarterly, 22(3), 358-379. doi: $10.1037 / 1045-3830.22 .3 .358$

Jones, K., Daley, D., Hutchings, J., Bywater, T., \& Eames, C. (2007). Efficacy of the incredible years basic parent training programme as an early intervention for children with conduct problems and ADHD. Child Care, Health, and Development, 33(6), 749-756. doi: 10.1111/j.1365-2214.2007.00747.x 
Kaminski, J. W., Valle, L. A., Filene, J. H., \& Boyle, C. L. (2008). A meta-analytic review of components associated with parent training program effectiveness. Journal of Abnormal Child Psychology, 36(4), 567-589. doi: 10.1007/s10802-007-9201-9

Kazdin, A. E. (1982). Single-case experimental designs in clinical research and practice. New Directions for Methodology of Social and Behavioral Science, 13, 33-47.

Klein, R. G., \& Abikoff, H. (1997). Behavior therapy and methylphenidate in the treatment of children with ADHD. Journal of Attention Disorders, 2(2), 89-114. doi: $10.1177 / 108705479700200203$

Klein, R. G., Abikoff, H., Hechtman, L., \& Weiss, G. (2004). Design and rationale of controlled study of long-term methylphenidate and multimodal psychosocial treatment in children with ADHD. Journal of the American Academy of Child and Adolescent Psychiatry, 43(7), 792-801. doi: 10.1097/01.chi.0000128798.91601.fe

Kratochvil, C. J., Wilens, T. E., Greenhill, L. L., Gao, H., Baker, K. D., Feldman, P. D., ... Gelowitz, D. L. (2006). Effects of long-term atomoxetine treatment for young children with attention-deficit/hyperactivity disorder. Journal of the American Academy of Child and Adolescent Psychiatry, 45(8), 919-927. doi: 10.1097/01.chi.0000222788.34229.68

Lahey, B. B., Pelham, W. E., Loney, J., Kipp, H., Ehrhardt, A., Lee, S. S., ...Massetti, G . (2004). Three-year predictive validity of DSM-IV attention deficit hyperactivity disorder in children diagnosed at 4-6 years of age. American Journal of Psychiatry, 161(11), 2014-2020. doi: 10.1176/appi.ajp.161.11.2014

Lahey, B. B., Pelham, W. E., Stein, M. A., Loney, J., Trepani, C., Nugent, K., ... Baumann, B. (1998). Validity of DSM-IV attention deficit/hyperactivity disorder for younger children. 
Journal of the American Academy of Child \& Adolescent Psychiatry, 37(7), 695-702. doi: 10.1097/00004583-199807000-00009

Lavigne, J. V., Gibbons, R. D., Christoffel, K. K., Arend, R., Rosenbaum, D., Binns, H., ...Isaacs, C. (1996). Prevalence rates and correlates of psychiatric disorders among preschool children. Journal of the American Academy of Child and Adolescent Psychiatry, 35(2), 204-214. doi: 10.1097/00004583-199602000-00014

Lavigne, J. V., LeBailly, S. A., Hopkins, J., Gouze, K. R., \& Binns, H. J. (2009). The prevalence of ADHD, ODD, depression, and anxiety in a community sample of 4-yearolds. Journal of Clinical Child and Adolescent Psychology, 38(3), 315-328. doi: $10.1080 / 15374410902851382$

Lemaster, K. B., Wagner, S. M., Tempel, A. B., \& McNeil, C. B. (2010). A preliminary examination of the effects of behavioral descriptions on on-task behavior. Psi Chi: Journal of Undergraduate Research, 15(2), 106-113.

Lerner, M., \& Wigal, T. (2008). Long-term safety of stimulant medications used to treat children with ADHD. Psychiatric Annals, 38(1), 43-51. doi: 10.3928/0048571320080101-10

Lenze, S. N., Pautsch, J., \& Luby, J. (2011). Parent-child interaction therapy emotional development: A novel treatment for depression in preschool children. Depression and Anxiety, 28, 153-159.

Lynam, D. R. (1996). Early identification of chronic offenders: Who is the fledgling psychopath? Psychological Bulletin, 120(2), 209-234. doi: 10.1037/00332909.120.2.209 
Mash, E. J., \& Johnston, C. (1982). A comparison of mother-child interactions of younger and older hyperactive and normal children. Child Development, 53(5), 1371-1381. doi: 10.1111/j.1467-8624.1982.tb04177.x

Mash, E. J., \& Johnston, C. (1983). Parental perceptions of child behavior problems, parenting self-esteem, and mothers' reported stress in younger and older hyperactive and normal children. Journal of Consulting and Clinical Psychology, 51(1), 86-99. doi: 10.1037//0022-006X.51.1.86

Masse, J. J. (2009). Examining the efficacy of parent-child interaction therapy with highfunctioning autism. Unpublished doctoral dissertation, West Virginia University.

Masse, J. J., \& McNeil, C. B. (2008). In-home parent-child interaction therapy: Clinical considerations. Child and Family Behavior Therapy, 30(2), 127-135. doi: $10.1080 / 07317100802060310$

Masse, J. J., McNeil, C. B., Wagner, S. M., \& Chorney, D. B. (2008). Parent-child interaction therapy and high functioning autism: A conceptual overview. Journal of Early and Intensive Behavior Intervention, 4(4), 714-735. Retrieved from http://www.jeibi.com/

Matos, M., Bauermeister, J. J., \& Bernal, G. (2009). Parent-child interaction therapy for Puerto Rican preschool children with ADHD and behavior problems: A pilot efficacy study. Family Process, 48(2), 232-252. doi: 10.1111/j.1545-5300.2009.01279.x

Matos, M., Torres, R., Santiago, R., Jurado, M., \& Rodriguez, I. (2006). Adaptation of parentchild interaction therapy for Puerto Rican families: A preliminary study. Family Processes, 45(2), 205-222. doi: 10.1111/j.1545-5300.2006.00091.x 
McCabe, K., \& Yeh, M. (2009). Parent-child interaction therapy for Mexican Americans: A randomized clinical trial. Journal of Clinical Child and Adolescent Psychology, 38(5), 753-759. doi: 10.1080/15374410903103544

McGoey, K. E., Eckert, T. L., \& DuPaul, G. J. (2002). Early intervention for preschool-age children with ADHD: A literature review. Journal of Emotional and Behavioral Disorders, 10(1), 14-28. doi: 10.1177/106342660201000103

McNeil, C. B., Capage, L. C., Bahl, A., \& Blanc, H. (1999). Importance of early intervention for disruptive behavior problems: Comparison of treatment and waitlist-control groups. Early Education and Development, 10(4), 445-454. doi: 10.1207/s15566935eed1004

McNeil, C. B., Clemens-Mowrer, L., Gurwith, R. H., \& Funderburk, B. W. (1994). Assessment of a new procedure to prevent timeout escape in preschoolers. Child and Family Behavior Therapy, 16(3), 27-35. doi: 10.1300/J019v16n03

McNeil, C. B., Eyberg, S., Eisenstadt, T. H., Newcomb, K., \& Funderburk, B. (1991). Parentchild interaction therapy with behavior problem children: Generalization of treatment effects to the school setting. Journal of Clinical Child Psychology, 20(2), 140-151. doi: $10.1207 / \mathrm{s} 15374424 \mathrm{jccp} 2002 \_5$

McNeil, C. B., Filcheck, H. A., Greco, L. A., Ware, L. M., \& Bernard, R. S. (2001). Parentchild interaction therapy: Can a manualized treatment be functional? The Behavior Analyist, 2(2), 106-114. Retrieved from: http://www.baojournal.com/

McNeil, C. B., \& Hembree-Kigin, T. L. (2010). Parent-child interaction therapy: Second edition. New York: Springer.

Molina, B. S. G., Hinshaw, S. P., Swanson, J. M., Arnold, L. E., Vittiello, B., Jensen, P. S., ...The MTA Cooperative Group. (2009). The MTA at 8 years: prospective follow-up of 
children treated for combined-type ADHD in a multsite study. Journal of the American Academy of Child and Adolescent Psychiatry, 48(5), 484-500. doi:

10.1097/CHI.0b013e31819c23d0

Murphy, K., \& Barkley, R. A. (1996). Attention deficit hyperactivity disorder adults: Comorbidities and adaptive impairments. Comprehensive Psychiatry, 37(6), 393-401. doi: 10.1016/S0010-440X(96)90022-X

Nixon, R. D. (2001). Changes in hyperactivity and temperament in behaviourally disturbed preschoolers after parent-child interaction therapy (PCIT). Behaviour Change, 18(3), 168-176. doi: 10.1375/bech.18.3.168

Owens, E. B., Hinshaw, S. P., Kraemer, H. C., Arnold, L. E., Abikoff, H. B., Cantwell, D. P., ...Wigal, T. (2003). Which treatment for whom for ADHD? Moderators of treatment response in the MTA. Journal of Consulting and Clinical Psychology, 71(3), 540-552. doi: 10.1037/0022-006X.71.3.540

Pappadopulos, E., Jensen, P. S., Chait, A. R., Arnold, E., Swanson, J. M., Greenhill, L. L., ... Newcorn, J. H. (2009). Medication adherence in the MTA: Saliva methylphenidate samples versus parent report and mediating effects of concomitant behavioral treatment. Journal of the American Academy of Child and Adolescent Psychiatry, 48(5), 501-510. doi: 10.1097/CHI.0b013e31819c23ed

Pavuluri, M. N., Luk, S., McGee, R. (1996). Help-seeking for behavior problems by parents of preschool children: A community study. Journal of the American Academy of Child and Adolescent Psychiatry, 35(2), 215-222. doi: 10.1097/00004583-199602000-00015 
Pelham, W. E. (1999). The NIMH multimodal treatment study for attention-deficit hyperactivity disorder: Just say yes to drugs alone? Canadian Journal of Psychiatry, 44(10), 481-490.

Pelham, W. E., Chacko, A., \& Wymbs, B. (2004). Diagnostic and assessment issues of attention deficit/hyperactivity disorder in the young child. In R. Wiggins and A. Carter (Eds.), Handbook of infant, toddler, and preschool mental health assessment (pp. 399-420). New York: Oxford University Press.

Pelham, W. E., \& Fabiano, G. A. (2008). Evidence-based psychosocial treatments for attentiondeficit/hyperactivity disorder. Journal of Clinical Child and Adolescent Psychology, 37(1), 184-214. doi: 10.1080/15374410701818681

Pelham, W. E., Fabiano, G. A., \& Massetti, G. M. (2005). Evidence-based assessment of attention deficit hyperactivity disorder in children and adolescents. Journal of Clinical Child and Adolescent Psychology, 34(3), 449-476. doi: 10.1207/s15374424jccp3403

Pelham, W. E., Gnagy, E. M., Greenslade, K. E., \& Milch, R. (1992). Teacher ratings of DSMIII-R symptoms of the disruptive behavior disorders. Journal of the American Academy of Child and Adolescent Psychiatry, 31(2), 210-218. doi: 10.1097/00004583-19920300000006

Pelham, W. E., Wheeler, T., \& Chronis, A. (1998). Empirically supported psychosocial treatments for attention deficit hyperactivity disorder. Journal of Clinical Child Psychology, 27(2), 190-205. doi: 10.1207/s15374424jccp2702_6

Pfiffner, L. J., \& McBurnnett, K. (1997). Social skills training with parent generalization: Treatment effects for children with attention deficit disorder. Journal of Consulting and Clinical Psychology, 65(5), 749-757. doi: 10.1037/0022-006X.65.5.749 
Pierce, E. W., Ewing, L. J., \& Campbell, S. B. (1999). Diagnostic status and symptomatic behavior of hard-to-manage preschool children in middle childhood and early adolescence. Journal of Clinical Child Psychology, 28(1), 44-67. doi: 10.1207/s15374424jccp2801_4

Pincus, D. B., Eyberg, S. M., \& Choate, M. L. (2005). Adapting parent-child interaction therapy for young children with separation anxiety disorder. Education and Treatment of Children, 28(2), 163-181. Retrieved from: http://www.educationandtreatmentofchildren.net/

Pisterman, S., McGrath, P., Firestone, P., Goodman, J. T., Webster, I., \& Mallory, R. (1989). Outcome of parent-mediated treatment of preschoolers with attention deficit disorder with hyperactivity. Journal of Consulting and Clinical Psychology, 57(5), 628-635. doi: 10.1037/0022-006X.57.5.628

Pliszka, S. R. (2007). Pharmacologic treatment of attention-deficit/hyperactivity disorder: Efficacy, safety, and mechanisms of action. Neuropsychology Review, 17(1), 61-72. doi: $10.1007 / \mathrm{s} 11065-006-9017-3$

Rayfield, A., \& Sobel, A. (2000). Effectiveness of "in-room" coaching of Parent-Child Interaction Therapy. Paper presented at the First Annual Parent-Child Interaction Therapy Conference, Sacramento, CA.

Reynolds, C. R., \& Kamphaus, R. W. (1998). BASC Behavior Assessment System for Children Manual. Circle Pines, MN: American Guidance Services Inc.

Rosenthal, J H. (1973). Neurophysiology of minimal cerebral dysfunction. Academic Therapy, 8(3), 291-294. doi: $10.1177 / 105345127300800305$ 
Satterfield, J. H., Faller, K. J., Crinella, F. M., Schell, A. M., Swanson, J. M., \& Homer, L. D. (2007). A 30-year prospective follow-up study of hyperactive boys with conduct problems: Adult criminality. Journal of the American Academy of Child and Adolescent Psychiatry, 46(5), 601-610. doi: 10.1097/chi.0b013e318033ff59

Shaffer, D., Fisher, P. Lucas, C. P., Dulcan, M. K., \& Schwab-Stone, M. E. (2000). NIMH diagnostic interview schedule for children version IV (NIMH DISC-IV): Description, differences from previous versions, and reliability of some common diagnoses. Journal of the American Academy of Child and Adolescent Psychiatry, 39(1), 28-38. doi:10.1097/00004583-200001000-00014

Schuhmann, E. M., Foote, R. C., Eyberg, S. M., Boggs, S. R., \& Algina, J. (1998). Efficacy of parent-child interaction therapy: Interim report of a randomized trial with short-term maintenance. Journal of Clinical Child Psychology, 27(1), 34-45. doi: $10.1207 / \mathrm{s} 15374424 \mathrm{jccp} 2701 \_4$

Shanley, J. R., \& Niec, L. N. (2010). Coaching parents to change: The impact of in vivo feedback on parents' acquisition of skills. Journal of Clinical and Adolescent Psychology, 39(2), 282-287. doi: 10.1080/15374410903532627

Sheridan, S. M., Dee, C. C., Morgan, J. C., McCormick, M. E., \& Walker, D. (1996). A multimethod intervention for social skills deficits in children with ADHD and their parents. School Psychology Review, 25(1), 57-76. Retrieved from: http://www.nasponline.org/publications/spr/sprmain.aspx

Slifer, K. J., Kruglak, D., Benore, E., Bellipanni, K., Falk, L., Halbower, A. C., .. Beck, M. (2007). Behavioral training for increasing preschool children's adherence with positive 
airway pressure: A preliminary study. Behavioral Sleep Medicine, 5, 147-175. Retrieved from: http://www.tandf.co.uk/journals/HBSM

Sokol, N. G., Kern, L., Arbolino, L. A., Thomas, L. B., \& DuPaul, G. J. (2009). A summary of home-based functional analysis data for young children with or at risk for attentiondeficit/hyperactivity disorder. Early Childhood Services: An Interdisciplinary Journal of Effectiveness, 3(2), 127-142.

Sonuga-Barke, E. J., Daley, D., Thompson, M., Laver-Bradbury, C., \& Weeks, A. (2001). Parent-based therapies for preschool attention-deficit/hyperactivity disorder: A randomized, controlled trial with a community sample. Journal of the American Academy of Child and Adolescent Psychiatry, 40(4), 402-408. doi: 10.1097/00004583200104000-00008

Strayhorn, J. M., \& Weidman, C. S. (1989). Reduction of attention deficit and internalizing symptoms in preschoolers through parent-child interaction training. Journal of the American Academy of Child and Adolescent Psychiatry, 28(6), 889-896. doi: $10.1097 / 00004583-198911000-00013$

Strayhorn, J.M., \& Weidman, C.S. (1991). Follow-up year after parent-child interaction training: Effects on behavior of preschool children. Journal of the American Academy of Child and Adolescent Psychiatry, 30(1), 138-143.

Swanson, J., Greenhill, L., Wigal, T., Kollins, S., Stehli, A., Davies, M.,... Wigal, S. (2006). Stimulant-related reductions of growth rates in the PATS. Journal of the American Academy of Child and Adolescent Psychiatry, 45(11), 1304-1313. doi: 10.1097/01.chi.0000235075.25038.5a 
Swanson, J. M., Kraemer, H. C., Hinshaw, S. P., Arnold, L. E., Conners, C. K., Abikoff, H. B., ... Wu, M. (2001). Clinical relevance of the primary findings of the MTA: Success rates based on severity of ADHD and ODD symptoms at the end of treatment. Journal of the American Academy of Child and Adolescent Psychiatry, 40(2), 168-179. doi:

$10.1097 / 00004583-200102000-00011$

Tempel, A. B. (2009). Parent-child interaction therapy: The effects of parental attention components on children's verbalizations and attending-to-task behaviors. Unpublished master's thesis, West Virginia University.

The MTA Cooperative Group. (1999). A 14-month randomized clinical trial of treatment strategies for attention-deficit/hyperactivity disorder. Archives of General Psychiatry, 56(12), 1073-1086. doi: 10.1001/archpsyc.56.12.1073

The MTA Cooperative Group. (2004). The NIMH MTA follow-up: 24-month outcomes of treatment strategies for attention-deficit/hyperactivity disorder (ADHD). Pediatrics, 113(4), 754-761. Retrieved from: http://pediatrics.aappublications.org/

Thomas, R. \& Zimmer-Gembeck, M. J. (2007) Behavioral outcomes of parent-child interaction therapy and triple p-positive parenting program: A review and meta-analysis. Journal of Abnormal Child Psychology, 35(3), 475-495. doi: 10.1007/s10802-007-9104-9

Timmer, S. G., Zebell, N. M., Culver, M. A., \& Urquiza, A. J. (2010). Efficacy of adjunct inhome coaching to improve outcomes in parent-child interaction therapy. Research on Social Work Practice, 20(1), 36-45. doi: 10.1177/1049731509332842

Todd, R. D., Huang, H., Todorov, A. A., Neuman, R. J., Reiersen, A. M., Henderson, C. A., \& Reich, W. C. (2008). Predictors of stability of attention-deficit/hyperactivity disorder 
subtypes from childhood to young adulthood. Journal of the American Academy of Child and Adolescent Psychiatry, 47(1), 76-85. doi: 10.1097/chi.0b013e31815a6aca

Treacy, L., Tripp, G., \& Baird, G. (2005). Parent stress management training for attentiondeficit/hyperactivity disorder. Behavior Therapy, 36(3), 223-233. doi: 10.1016/S00057894(05)80071-1

Van Cleave, J., \& Leslie, L. K. (2008). Approaching ADHD as a chronic condition: Implications for long-term adherence. Journal of Psychosocial Nursing, 46(8), 28-37. doi: $10.3928 / 00904481-20080101-07$

van den Hoofdakker, B. J., van der Veenn-Mulders, L., Sytema, S., Emmelkamp, P. M. G., Minderaa, R. B., \& Nauta, M. H. (2007). Effectiveness of behavioral parent training for children with ADHD in routine clinical practice: A randomized controlled study. Journal of the American Academy of Child and Adolescent Psychiatry, 46(10), 1263-1271. doi: 10.1097/chi.0b013e3181354bc2

Verduin, T. L., Abikoff, H., \& Kurtz, S. M. (2008). Evidence-based treatment of attention deficit/ hyperactivity disorder in a preschool-age child: A case study. Journal of Clinical Child and Adolescent Psychology, 37(2), 477-485. doi: 10.1080/15374410801955904 Vitiello, B., Severe, J. B., Greenhill, L. L., Arnold, L. E., Abikoff, H. B., Bukstein, O. G., ,.. Cantwell, D. P. (2001). Methylphenidate dosage for children with ADHD over time under controlled conditions: Lessons from the MTA. Journal of the American Academy of Child and Adolescent Psychiatry, 40(2), 188-196. doi: 10.1097/00004583-20010200000013 
von Stauffenberg, C., \& Campbell, S. B. (2007). Predicting the early developmental course of symptoms of attention deficit hyperactivity disorder. Journal of Applied Developmental Psychology, 28(5-6), 536-552. doi: 10.1016/j.appdev.2007.06.011

Wagner, S. M., \& McNeil, C. B. (2008). Parent-child interaction therapy for ADHD: A conceptual overview and critical literature review. Child and Family Behavior Therapy, 30(3), 231-256. doi: 10.1080/07317100802275546

Ware, L. M., McNeil, C. B., Masse, J. J., \& Stevens, S. B. (2008). Efficacy of in-home parentchild interaction therapy. Child and Family Behavior Therapy, 30(2), 99-126. doi: $10.1080 / 07317100802060302$

Watson, P. J., \& Workman, E. A. (1981). The non-concurrent multiple baseline acrossindividuals design: An extension of the traditional multiple baseline design. Journal of Behavior Therapy and Experimental Psychiatry, 12(3), 257-259. doi: 10.1016/00057916(81)90055-0

Webster-Stratton, C., \& Hammond, M. (1997). Treating children with early-onset conduct problems: A comparison of child and parent training interventions. Journal of Consulting and Clinical Psychology, 65(1), 93-109. doi: 10.1037/0022-006X.65.1.93

Weiss, G., \& Hechtman, L. T. (1993). Hyperactive children grown-up. New York: Guilford Press.

Wells, K. C., Epstein, J. N., Hinshaw, S. P., Conners, K. C., Klaric, J., Abikoff, H. B., ...Wigal, T. (2000). Parenting and family stress treatment outcomes in attention deficit hyperactivity disorder (ADHD): An empirical analysis in the MTA study. Journal of Abnormal Child Psychology, 28(6), 543-553. doi: 10.1023/A:1005131131159 
Wells, K. C., Pelham, W. E., Kotkin, R. A., Hoza, B., Abikoff, H. B., Abramowitz, A., ...Schiller, E. (2000). Psychosocial treatment strategies in the MTA study: Rationale, methods, and critical issues in design and implementation. Journal of Abnormal Child Psychology, 28(6), 483-505. doi: 10.1023/A:1005174913412

Werry, J. S. (1968). Developmental hyperactivity. Pediatric Clinics of North America, 15, 584-599.

Whalen, C. K., \& Henker, B. (1985). The social worlds of hyperactive (ADDH) children. Clinical Psychology Review, 5(5), 447-478. doi: 10.1016/0272-7358(85)90004-2

Wilder, D. A., \& Atwell, J. (2006). Evaluation of a guided compliance procedure to reduce noncompliance among preschool children. Behavioral Interventions, 21(4), 265-272. doi: 10.1002/bin.222

Wolraich, M. L., McGuinn, L., \& Doffing, M. (2007). Treatment of attention deficit hyperactivity disorder in children and adolescents: Safety considerations. Drug Safety, 30(1), 17-26. Retrieved from: http://www.isoponline.org/drug-safety.html

Zentall, S. S. (2005). Theory- and evidence-based strategies for children with attentional problems. Psychology in the Schools, 4(8), 821-836. doi: 10.1002/pits.20114 
Appendix A

Definitions of DPICS-III Codes for the Study 


\begin{tabular}{|l|l|}
\hline \multicolumn{1}{|c|}{ Code } & \multicolumn{1}{|c|}{ Definition } \\
\hline Behavioral Description & $\begin{array}{l}\text { Descriptive statements in which the subject of the } \\
\text { sentence is the child and the verb describes the child's } \\
\text { ongoing or immediately completed (<5 seconds) } \\
\text { verbal or nonverbal observable behavior. }\end{array}$ \\
\hline Labeled Praise & $\begin{array}{l}\text { Labeled praise provides a positive evaluation of a } \\
\text { specific behavior, activity, or product of the child. }\end{array}$ \\
\hline Reflection & $\begin{array}{l}\text { A declarative phrase or statement that has the same } \\
\text { meaning as an immediately preceding child } \\
\text { verbalization. The reflection may paraphrase or } \\
\text { elaborate upon the child's verbalization but may not } \\
\text { change the meaning of the child's statement or } \\
\text { interpret unstated ideas. }\end{array}$ \\
\hline Direct Command & $\begin{array}{l}\text { performed and indicate that the child is to perform this } \\
\text { behavior. } \\
\text { performed that is implied or stated in question form. }\end{array}$ \\
\hline
\end{tabular}




\begin{tabular}{|c|c|}
\hline Negative Talk & $\begin{array}{l}\text { A verbal expression of disapproval for the child or the } \\
\text { child's attributes, products, or choices. Negative talk } \\
\text { also includes sassy, sarcastic, rude, or impudent } \\
\text { speech. }\end{array}$ \\
\hline Question & $\begin{array}{l}\text { A descriptive or reflective comment or } \\
\text { acknowledgement expressed in question form. }\end{array}$ \\
\hline Child Compliance & $\begin{array}{l}\text { Coded when the child obeys or begins to obey the } \\
\text { command within the } 5 \text {-second interval. }\end{array}$ \\
\hline Child Noncompliance & $\begin{array}{l}\text { Coded following a direct or indirect parental command } \\
\text { when the child does not obey, attempt to obey, or stops } \\
\text { attempting to complete the requested behavior within } \\
\text { the } 5 \text {-second interval following the command. }\end{array}$ \\
\hline $\begin{array}{l}\text { Child No Opportunity for } \\
\text { Compliance }\end{array}$ & $\begin{array}{l}\text { Coded when the child is not given an adequate chance } \\
\text { to comply with a command. }\end{array}$ \\
\hline
\end{tabular}


Appendix B

DPICS-III Situation Instructions 


\section{Instructions for the Three Standard Situations}

The directions are typically presented to the parent over a "bug-in-the-ear" microphone device from an observation room. In this way, the child hears the initial directions from the parent rather than from the therapist.

1) Child-Led Play

"In this situation tell_that he/she may play with whatever he/she chooses. Let him/her choose any activity he/she wishes. You just follow his/her lead and play along with him/her."

After the 5-minute warm-up period, the parent is told:

"You're doing a nice job of letting lead the play. Please continue to let him/her lead."

2) Parent-Led Play

"That was fine. Please do not clean up the toys at this time. Now we'll switch to the second situation. Tell that it is your turn to choose the game. You may choose any activity. Keep him/her playing with you according to your rules."

After the 5-minute PLP warm-up period, the parent is told:

"You're doing a nice job of leading the play. Please continue to get to play along with you according to your rules."

\section{3) Clean-Up}

"That was fine. Now please tell that it is time to leave the playroom and the toys must be put away. Make sure you have him/her put the toys away by him/herself. Have him/her put all the toys in their containers and all the containers in the toy box." 
Appendix C

DPICS-III Coding Sheet 


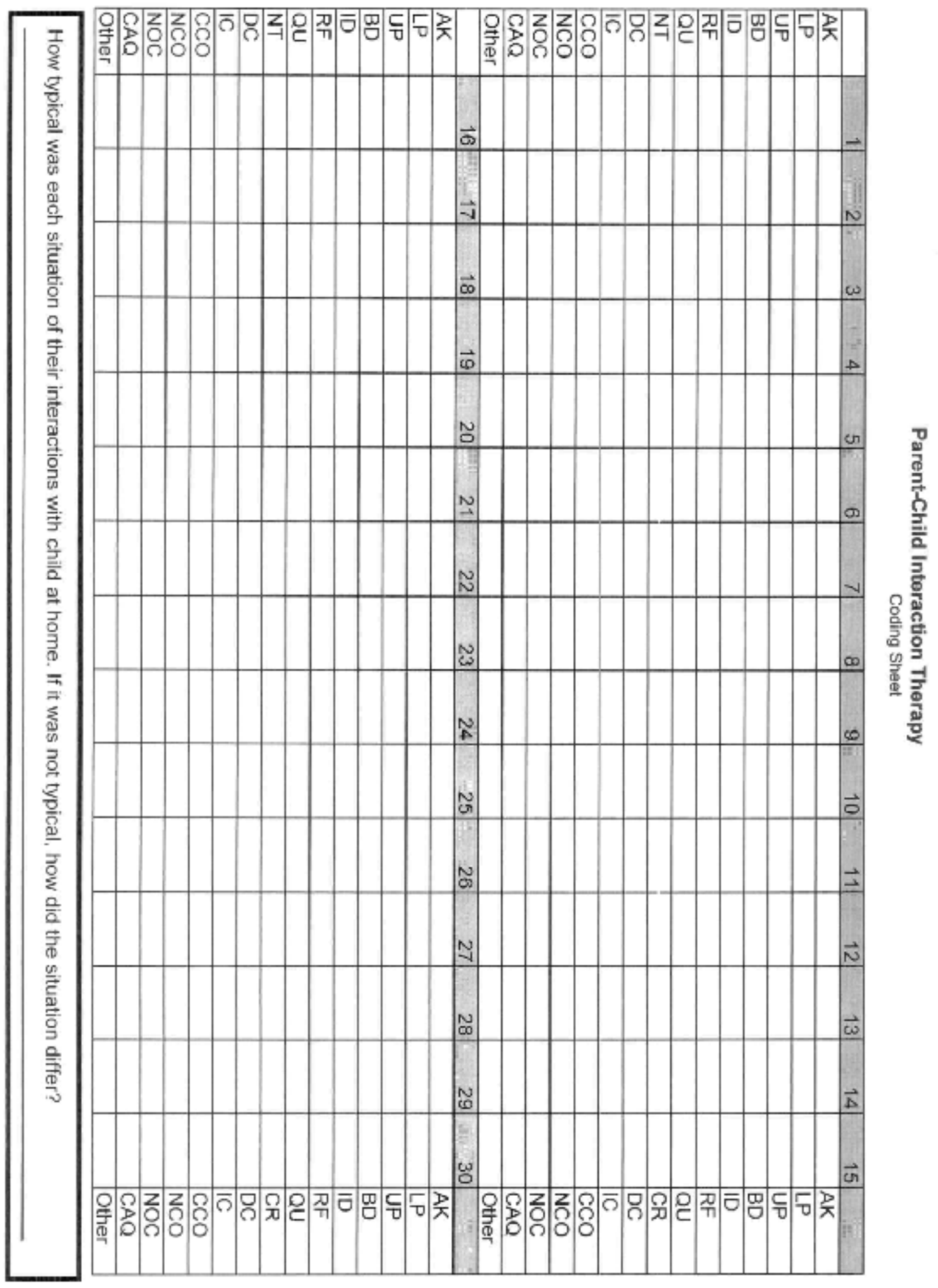


Appendix D

REDSOCS Instructions 
Instructions for Coloring Task

Set up mat, tray table coloring station, video camera (if pre, mid, post, or follow-up evaluation), audio tape of intervals and headphones, and coding sheets.

(Therapist reads instructions to parents prior to task but can abbreviate instructions once it is clear that the parents understand the instructions (e.g., 4 observation or so and after)

You and your child are going to work together on a coloring activity now. Please tell your child that he or she may use any of the paper and crayons that he or she wants. Let him or her know that he/she should keep coloring until you tell him/her to stop. You may color with him/her and answer any questions that pertain to coloring. You may use any parenting skills or discipline that you know to keep your child on task during this time.

After instructions, therapist hands parent 5 blank sheets of paper and 4 crayons ( 1 red, 1 green, 1 blue, and 1 yellow)

As soon as parent and child start task, start interval tape and begin coding. 
Appendix E

REDSOCS Record Form 


\section{REDSOCS Record Sheet}

Child ID:

Task:

Date:

Time:

Coder:

\begin{tabular}{|l|l|l|l|l|l|l|l|l|l|l|l|l|l|l|l|}
\hline Child \# _- & \multicolumn{10}{|c|}{} \\
\hline Interval & $\mathbf{1}$ & $\mathbf{2}$ & $\mathbf{3}$ & $\mathbf{4}$ & $\mathbf{5}$ & $\mathbf{6}$ & $\mathbf{7}$ & $\mathbf{8}$ & $\mathbf{9}$ & $\mathbf{1 0}$ & $\mathbf{1 1}$ & $\mathbf{1 2}$ & $\mathbf{1 3}$ & $\mathbf{1 4}$ & $\mathbf{1 5}$ \\
\hline On-Task & & & & & & & & & & & & & & & \\
\hline Off-Task & & & & & & & & & & & & & & & \\
\hline Interval & $\mathbf{1 6}$ & $\mathbf{1 7}$ & $\mathbf{1 8}$ & $\mathbf{1 9}$ & $\mathbf{2 0}$ & $\mathbf{2 1}$ & $\mathbf{2 2}$ & $\mathbf{2 3}$ & $\mathbf{2 4}$ & $\mathbf{2 5}$ & $\mathbf{2 6}$ & $\mathbf{2 7}$ & $\mathbf{2 8}$ & $\mathbf{2 9}$ & $\mathbf{3 0}$ \\
\hline On-Task & & & & & & & & & & & & & & & \\
& & & & & & & & & & & & & & & \\
\hline
\end{tabular}


Appendix F

Sample Treatment Integrity Form 


\section{FIRST CDI COACHING SESSION}

Date:

Therapist:

Child:

\section{Integrity Check? Y / N Rater:}

\section{Materials to bring to this session:}

$\checkmark$ Participant's chart

$\checkmark$ Toy box

$\checkmark$ Clipboards

$\checkmark$ ECBI

$\checkmark$ Conners Parent Rating Scale

$\checkmark$ ECBI Graph (in chart)

$\checkmark$ Pencil for ECBI

$\checkmark$ Out of Area coding sheet

$\checkmark$ On-task coding sheet

$\checkmark$ DPICS coding sheets (1 for each coder in chart)

$\checkmark$ Interval audiotape, player, headphones

$\checkmark$ Timer for coding

$\checkmark$ Weekly Profile (in chart)

$\checkmark$ CDI homework sheet

$\checkmark$ Appointment card

\section{Goals of this session:}

* Strengthen rapport with the family

* Continue to provide support

* Reinforce the caregiver(s) for their use of the skills and their progress (remember, this session should only focus on the positive).

\section{TREATMENT SESSION}

\section{Check in and set up}

Have therapist administer ECBI and Conners' to caregiver.

While caregiver completes the forms, talk with child briefly about therapy

- Why they are in therapy

- What therapy will be like

- The room and the toys

- The in-room coaching rules (e.g., fcannot look at you or talk with you when you are playing with your mom. You should pretend like I'm invisible. I will be whispering ideas to your mom to make her play with you even more fun. When our coaching time is over, then I can talk with you and play with you again.") 
Set up PCIT area - place the toys in the play area (mat).

Remind caregiver of the Đo" (i.e., PRIDE) and Avoid" (i.e., commands, questions, criticisms) skills of CDI.

Review briefly, longer only if it seems you need to establish more credibility with the caregiver (i.e., if caregiver seems skeptical of treatment or of you as an expert). In this case, present this as a leading theory/approach in the area of child behavior problems, and emphasize how effective it is.

Ask for homework sheets. Review homework for about 10 minutes.

- If parents did not bring their homework sheets, or brought them back incompletely filled out, take their sheet (or new sheet) and fill in each day with them

- If parents have practiced every day, praise their conscientiousness and genuine efforts to help their child. Let them know you recognize the effort it takes to organize time and add a new event the routine.

- If the parents have not practiced every day, spend time teaching parents to problem-solve around this issue. Guide them to make a specific plan for when and where each day they will practice.

- When discussing homework activities on parents' sheets, note appropriateness of the activity/toys for CDI. Either praise their choices, or have them discuss how it worked to use that (inappropriate) activity. Then have them problem-solve until they achieve a solution.

- Comment on any notes parent wrote on homework sheet

Discuss one issue unrelated to the child's behavior or the caregiver's use of treatment skills or child management.

\section{Coding and coaching}

Note that today is the first session in which the new skills will be coded and coached. Describe the procedures.

$\square$ Prepare interval audiotape and have therapists put on headphones.

$\square$ Get into your coaching position next to the caregiver (away from the child). 
Read CDI instructions for caregiver as follows:

In this situation, tell $\boldsymbol{N A M E}$ that he/she may play with whatever he/she chooses. Let him/her choose any activity he/she wishes. Just follow his/her lead and play along with $\operatorname{him} /$ her."

Begin interval audiotape.

Set timer and code for exactly 5 minutes (Code out-of-area behavior during this time).

\section{Coding On-Task Observations}

Set up play area for the task (clear area of toys, place tray on mat, get out crayons and paper).

Explain to the caregiver the purpose of the assessment and read instructions:

You and your child are going to work together on a coloring activity now. Please tell your child that he or she may use any of the paper and crayons that he or she wants. Let him/her know that he/she should keep coloring until you tell him/her to stop. You may color with him/her and answer any questions. You may use any parenting skills or discipline that you know to keep your child on task during this time."

Code on task behavior using the coding sheet and interval audiotape

Coach parent with child for about 30 minutes.

a) Have caregiver tell child the rules of special playtime.

Foday we are going to have special playtime. You can play with any of the toys on the mat, and I will play with you. There are two rules. You have to play gently with the toys and you have to stay on the mat. If you play roughly or get off of the mat, I will turn around like this and play all by myself. Then, when you play nicely or sit down, I will turn back around and play with you. You're playing nicely now, so we can play with anything on this table that you want to play with."

b) Give caregiver labeled praises for the best skills demonstrated during coding.

c) Focus coaching primarily on behavioral descriptions

d) Give only positive feedback today; don't point out mistakes. 


\section{Review}

Review coding sheets with caregiver for about five minutes.

Focus on their strengths and reassure them that they are doing fine.

\section{Wrap up}

$\square$ Give new CDI homework sheets and encourage caregiver to focus especially on decreasing questions and increasing reflections during their home practice. (Unless these skills are already at criterion and another skill needs more emphasis).

$\square$ Confirm next appointment time with caregiver and give caregiver appointment card reminder.

$\square$ File integrity checklist(s), coding sheet(s), homework sheet, and ECBI in participant's file.

\section{Intermediate tasks}

$\square$ Steph/Ashley/Andrea

- Enter DPICS, On-task, and Out-of-area data into Excel database.

- Examine data to determine if CDI criteria have been met.

- File participant chart.

Undergraduates/Graduate students

- Score ECBI and Conners

- Plot ECBI data on ECBI Graph (in chart).

- Plot DPICS data on Weekly Profile (in chart).

\section{Notes}

$\square$ Please note anything unusual that happened during today's session (or any deviations from protocol) here: 
Table 1

Treatment Length for Current Study and Past PCIT Research

Study

Sample/Context

Treatment Sessions

Wagner (2011)

ADHD/In-Home

21.5

Shuhmann et al. (1998)

DBD/Clinic

13

Timmer et al. (2005)

Maltreated children/Clinic

15.2

McCabe and Yeh (2009)

Hispanic/Clinic

18.7

Masse (2009)

Autism/In-Home

18.00

Note. Treatment sessions include both didactic and coding sessions and exclude baseline and assessment sessions. The number of sessions reflect additional sessions past mastery due to observed instability in on-task behavior. 
Table 2

Comparison of ECBI Intensity and Problem Scores to previous PCIT studies

\begin{tabular}{|c|c|c|c|c|c|c|c|}
\hline Study & Sample/Context & $\begin{array}{c}\text { Pre: I } \\
M(S D)\end{array}$ & $\begin{array}{l}\text { Pre: } \mathrm{P} \\
M(S D)\end{array}$ & $\begin{array}{l}\text { Post: I } \\
M(S D)\end{array}$ & $\begin{array}{l}\text { Post: P } \\
M(S D)\end{array}$ & $\begin{array}{c}\mathrm{F} / \mathrm{U}: \mathrm{I} \\
M(S D)\end{array}$ & $\begin{array}{l}\mathrm{F} / \mathrm{U}: \mathrm{P} \\
M(S D)\end{array}$ \\
\hline Wagner & $\mathrm{ADHD} /$ & 183.50 & 27.25 & 70.50 & 5.5 & 75.75 & 7.5 \\
\hline (2011) & In-Home & $(12.79)$ & $(1.26)$ & $(17.46)$ & $(3.79)$ & $(31.67)$ & $(9.26)$ \\
\hline $\begin{array}{l}\text { Nixon et al. } \\
(2003)\end{array}$ & ODD/Clinic & $\begin{array}{r}166.59 \\
(18.93)\end{array}$ & * & $\begin{array}{l}125.24 \\
(21.67)\end{array}$ & * & $\begin{array}{r}117.47 \\
(31.69)\end{array}$ & $*$ \\
\hline $\begin{array}{l}\text { Nixon et al. } \\
(2003)\end{array}$ & $\begin{array}{l}\text { ODD/Wait-list } \\
\text { Controls }\end{array}$ & $\begin{array}{r}173.82 \\
(22.72)\end{array}$ & $*$ & $\begin{array}{r}148.35 \\
(19.05)\end{array}$ & * & $*$ & $*$ \\
\hline $\begin{array}{l}\text { Schuhmann } \\
\text { et al. } \\
\text { (1998) }\end{array}$ & ODD PCIT/Clinic & $\begin{array}{r}170.3 \\
(26.4)\end{array}$ & $\begin{array}{l}21.9 \\
(6.5)\end{array}$ & $\begin{array}{r}117.6 \\
(40.4)\end{array}$ & $\begin{array}{r}10.9 \\
(9.6)\end{array}$ & $*$ & $*$ \\
\hline $\begin{array}{l}\text { Schuhmann } \\
\text { et al. } \\
\text { (1998) }\end{array}$ & $\begin{array}{l}\text { ODD/Wait-list } \\
\text { Controls }\end{array}$ & $\begin{array}{l}172.9 \\
(25.8)\end{array}$ & $\begin{array}{l}21.2 \\
(6.1)\end{array}$ & $\begin{array}{r}169.7 \\
(34.1)\end{array}$ & $\begin{array}{l}22.1 \\
(8.0)\end{array}$ & $*$ & $*$ \\
\hline $\begin{array}{l}\text { Ware } \\
(2006)\end{array}$ & $\begin{array}{l}\text { Disruptive Behavior/In- } \\
\text { Home }\end{array}$ & $\begin{array}{r}157.67 \\
(34.82)\end{array}$ & $*$ & $\begin{array}{r}89.33 \\
(12.90)\end{array}$ & * & $\begin{array}{r}71.67 \\
(8.14)\end{array}$ & $*$ \\
\hline
\end{tabular}

Note. ${ }^{*}=$ not reported or assessment not conducted. Pre $=$ Pre-treatment assessment; Post $=$ Post-treatment assessment; F/U $=$ Followup assessment; I = Intensity; P = Problem. 
Table 3

Comparison of consumer satisfaction to previous PCIT studies

\begin{tabular}{|c|c|c|c|c|}
\hline Study & Sample/Context & $\begin{array}{c}\text { Mid- } \\
\text { Treatment } \\
M(S D)\end{array}$ & $\begin{array}{c}\text { Post-Treatment } \\
M(S D)\end{array}$ & $\begin{array}{l}\text { Follow-Up } \\
M(S D)\end{array}$ \\
\hline $\begin{array}{l}\text { Wagner } \\
\text { (2011) }\end{array}$ & ADHD/In-Home & $40.45(1.71)$ & $47.75(2.87)$ & $46.05(5.07)$ \\
\hline $\begin{array}{l}\text { Schuhmann } \\
\text { et al. (1998) }\end{array}$ & ODD /Clinic & $*$ & $44.10(4.80)$ & * \\
\hline \multirow[t]{2}{*}{$\begin{array}{l}\text { Eisenstadt et } \\
\text { al. (1993) }\end{array}$} & $\begin{array}{l}\text { Externalizing } \\
\text { Disorder /CDI first } \\
\text { clinic }\end{array}$ & $39.50(5.80)$ & $45.80(3.60)$ & $*$ \\
\hline & $\begin{array}{l}\text { Externalizing } \\
\text { Disorder/PDI first } \\
\text { clinic }\end{array}$ & $44.60(6.60)$ & $49.60(0.70)$ & $*$ \\
\hline $\begin{array}{l}\text { Matos et al. } \\
(2009)\end{array}$ & $\begin{array}{l}\text { ADHD, Puerto Rican } \\
\text { Families/Clinic }\end{array}$ & $*$ & $47.80(2.93)$ & * \\
\hline $\begin{array}{l}\text { Matos et al. } \\
(2006)\end{array}$ & $\begin{array}{l}\text { ADHD symptoms, } \\
\text { Puerto Rican } \\
\text { Families/Clinic }\end{array}$ & $*$ & $48.67(1.32)$ & * \\
\hline Masse (2009) & Autism/In-Home & $*$ & $44.67(5.13)$ & $43.33(5.51)$ \\
\hline Ware (2006) & $\begin{array}{l}\text { Disruptive } \\
\text { Behavior/In-Home }\end{array}$ & $*$ & $48.00(3.46)$ & $48.33(2.87)$ \\
\hline
\end{tabular}

Note. $*=$ not reported or assessment not conducted. 


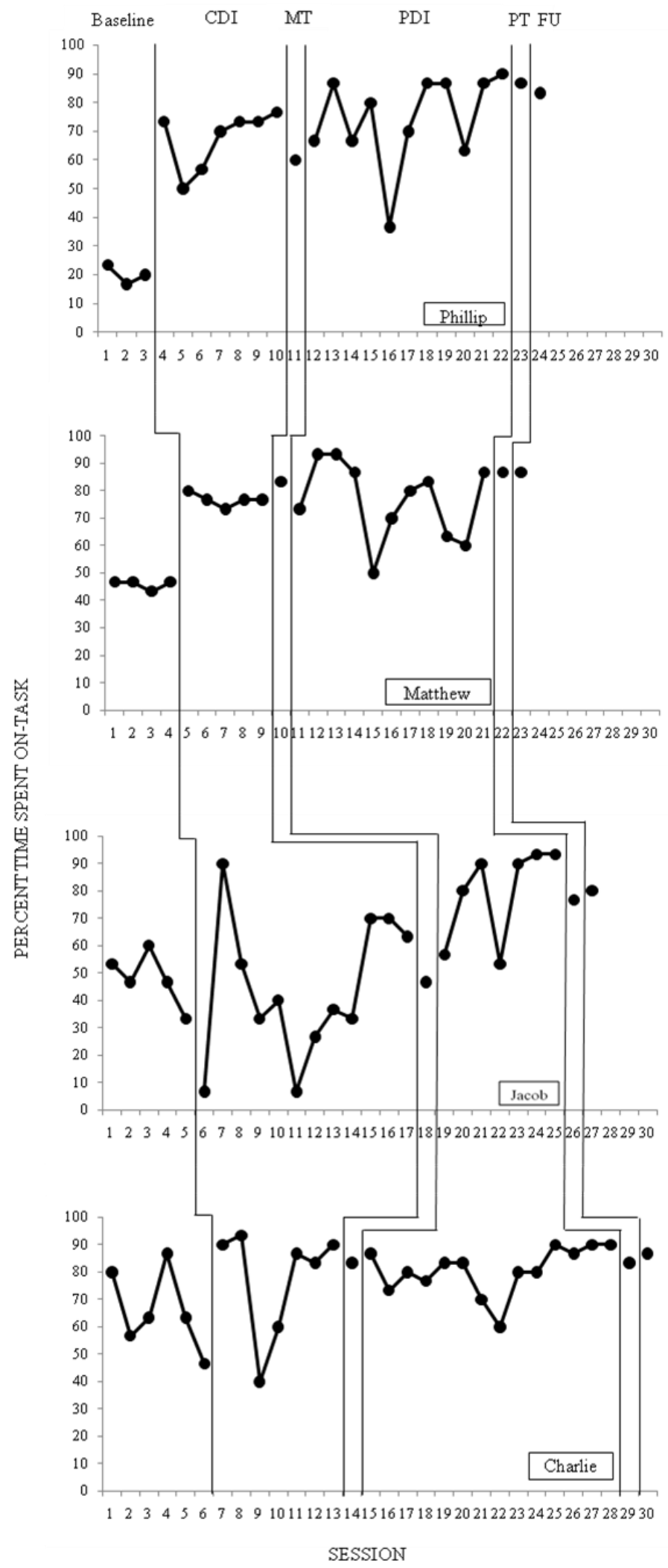

Figure 1. Percent of time spent on-task during coloring for each participant across phases. CDI $=$ Child Directed Interaction; MT = Mid-treatment; PDI = Parent Directed Interaction; PT = Post-treatment; FU = Follow-up. 


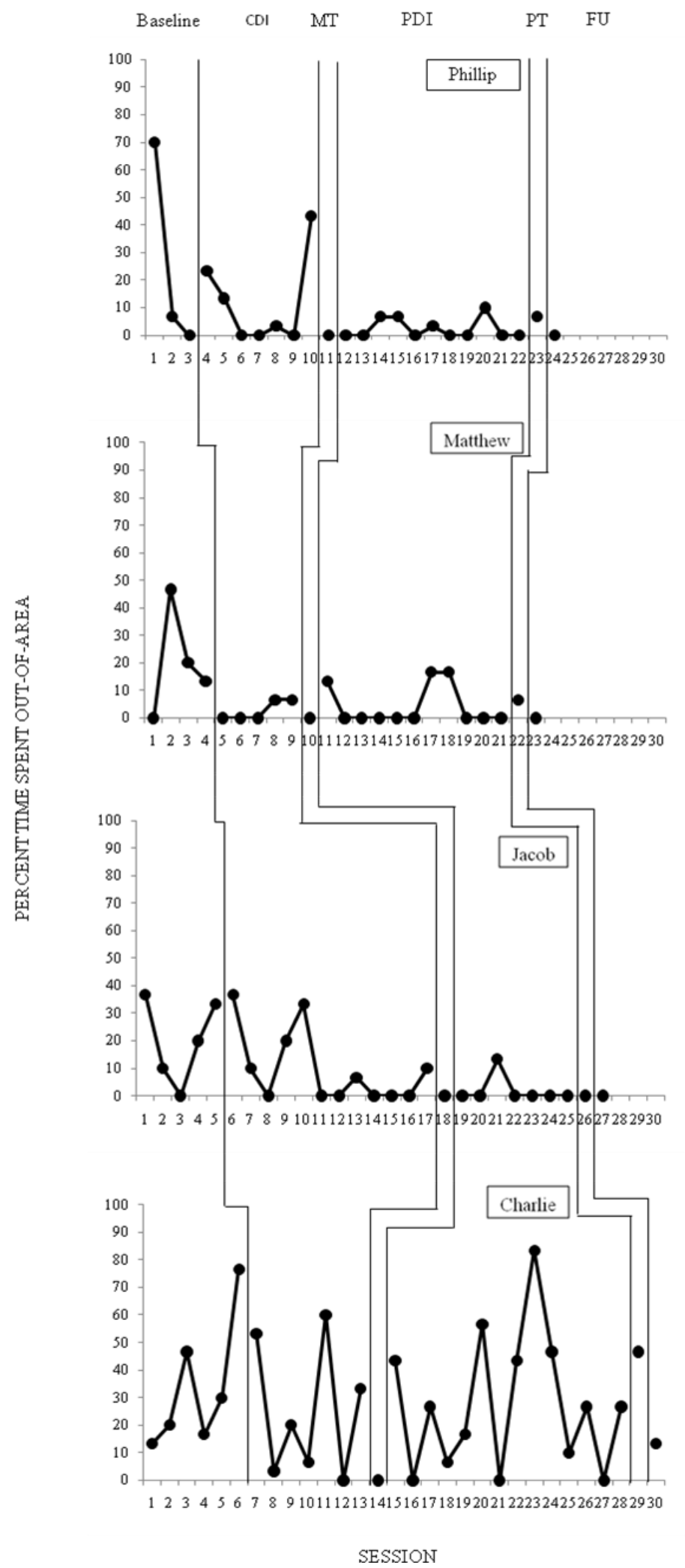

Figure 2. Percent of time spent out-of-area during CDI for each participant across phases. CDI $=$ Child Directed Interaction; MT = Mid-treatment; PDI = Parent Directed Interaction; PT = Post-treatment; FU = Follow-up. 

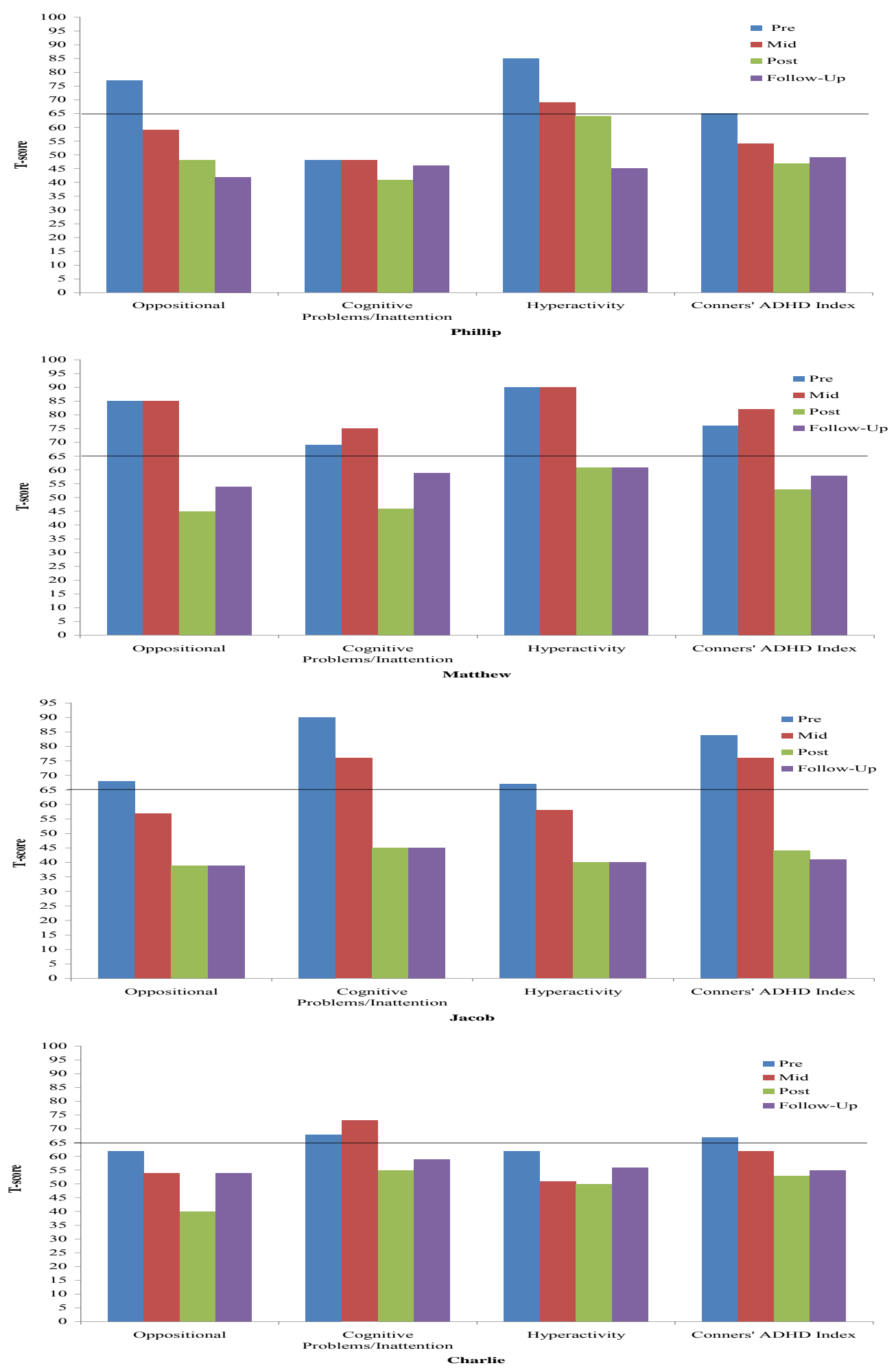

Figure 3. Conners' Parent Rating Scale - Revised Short (CPRS-RS) Subscales across participants and assessment points with horizontal lines indicating clinical cutoff. 

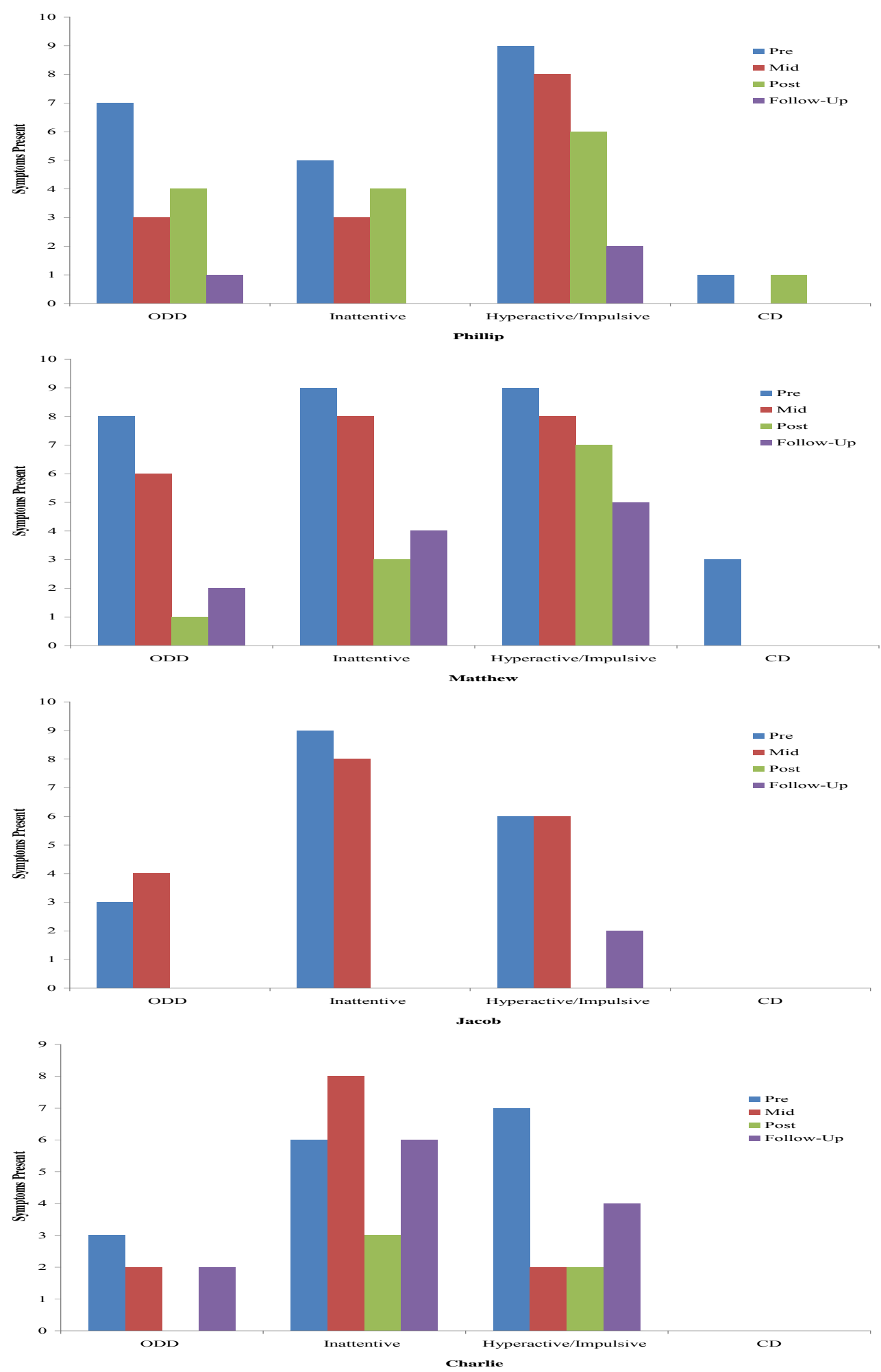

Figure 4. DSM-IV Diagnostic Interview for Disruptive Behavior Disorders Subscales across participants and assessment points. 

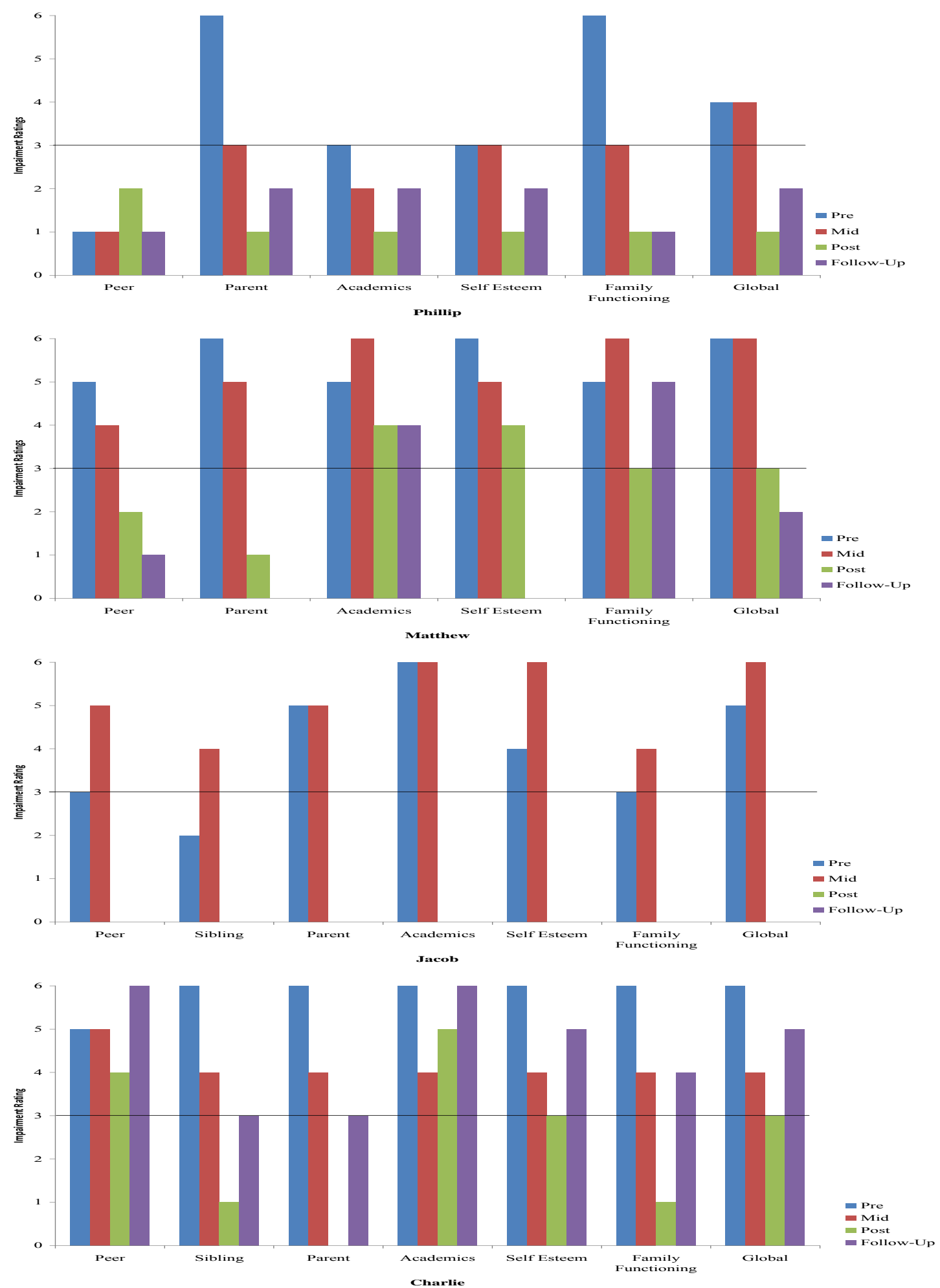

Figure 5. Impairment Rating Scale (IRS) Subscales across participants and assessment points with horizontal lines indicating clinical cutoff. 

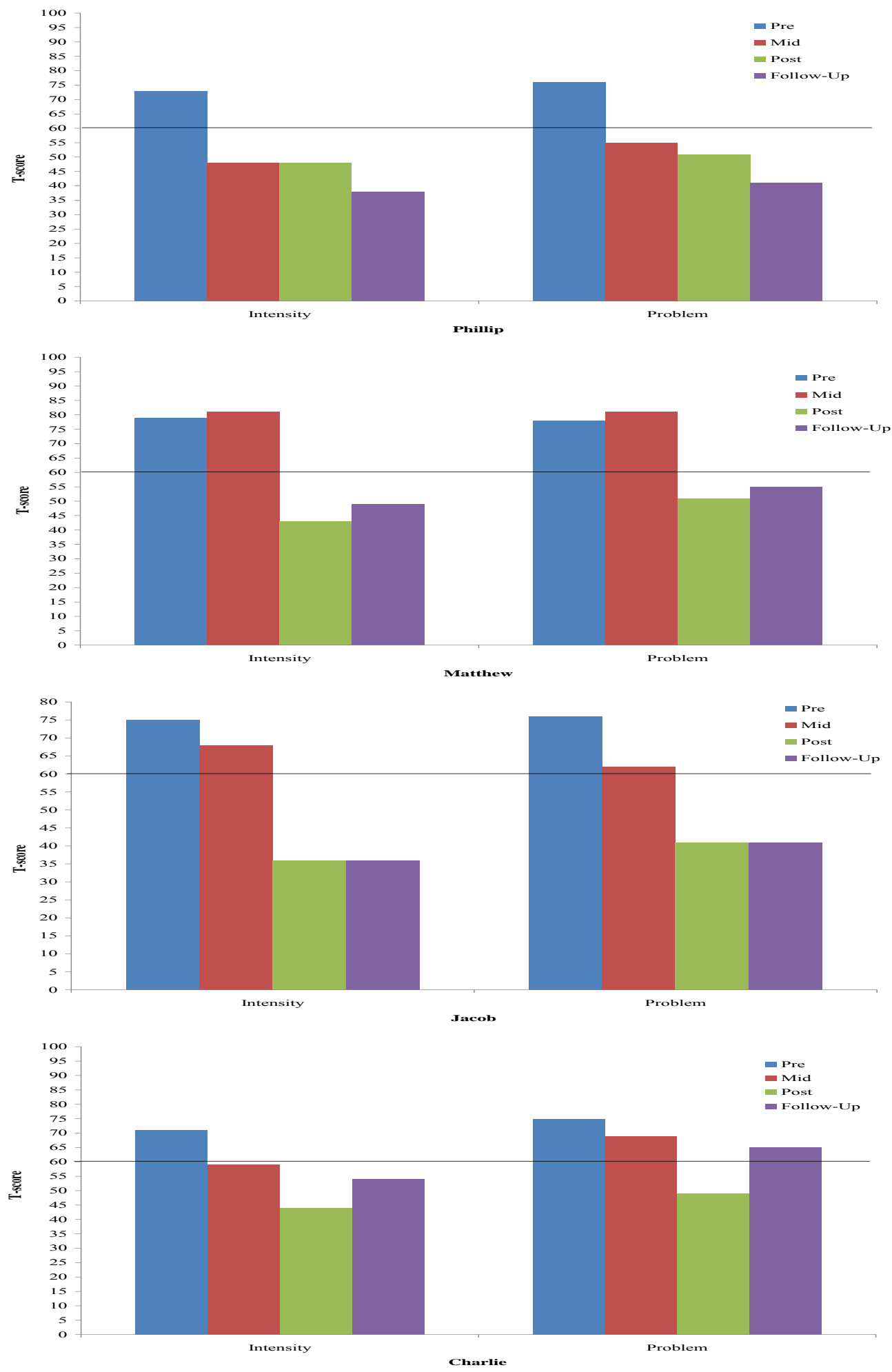

Figure 6. Eyberg Child Behavior Inventory (ECBI) Subscales across participants and assessment points with horizontal lines indicating clinical cutoff. 

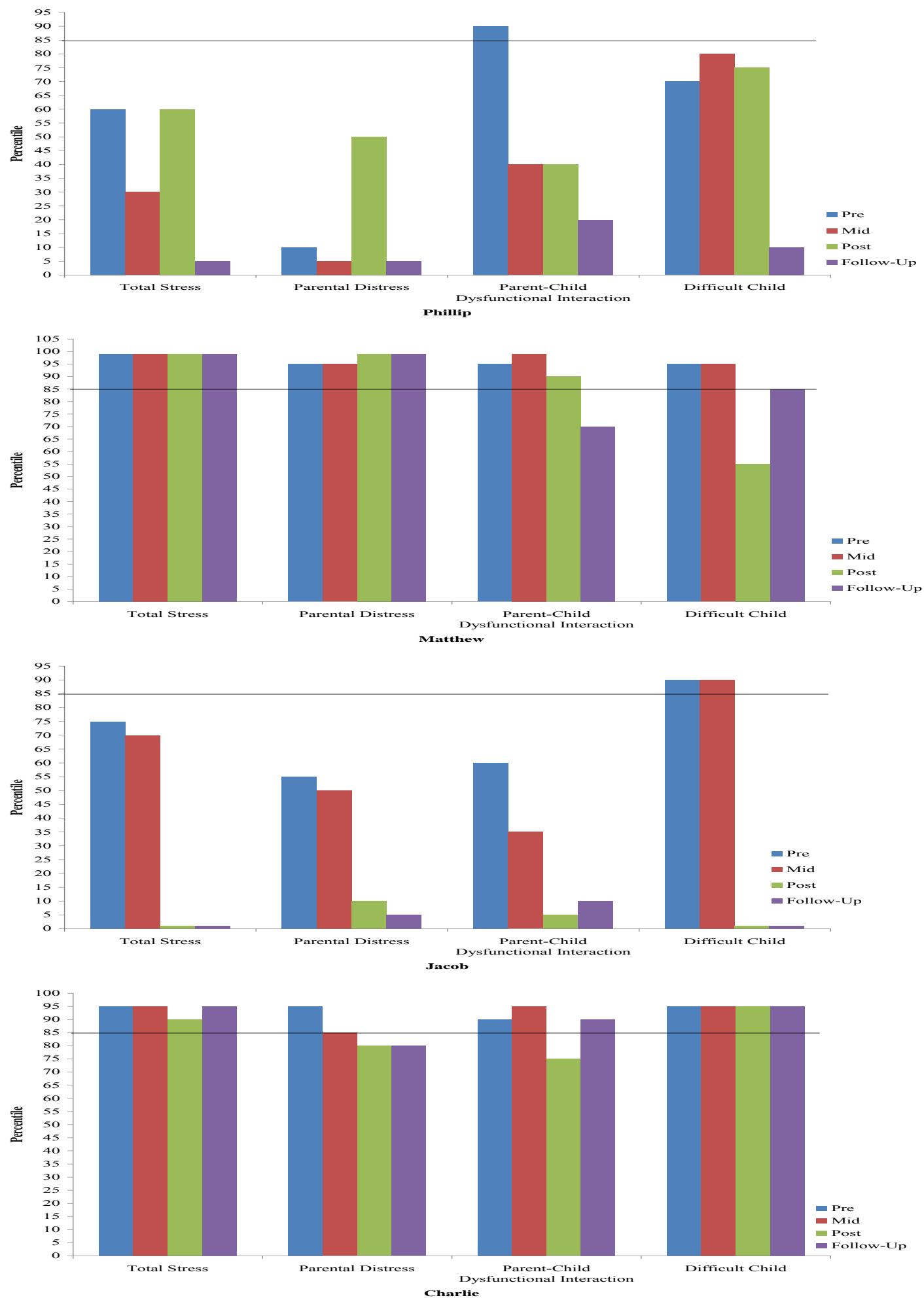

Figure 7. Parenting Stress Index - Short Form (PSI-SF) Overall Scale and Subscale scores across participants and phases with horizontal lines indicating clinical cutoff. 